\title{
Annual Report
}

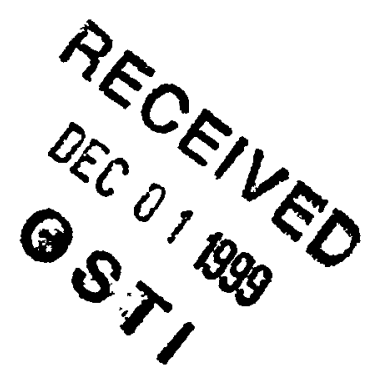

1 April 1995-31 March 1996

影研 Radiation Effects Research Foundation

RERF A Cooperative Japan-United States Research Organization 


\section{Annual Report}

\section{April 1995-31 March 1996}

\section{更影研 Radiation Effects Research Foundation \\ RERF A Cooperative Japan-United States Research Organization}


Publication date: January 1997

Publisher: Radiation Effects Research Foundation

\section{Hiroshima Laboratory}

5-2, Hijiyama Park, Minami-ku, Hiroshima

732 Japan

\section{Nagasaki Laboratory}

8-6, Nakagawa l-chome, Nagasaki

850 Japan

Inquiries: Publication \& Documentation Center, RERF Internet: pub-info@rerf.or.jp

Fax from outside Japan: 81-82-261-3197

Fax from inside Japan: 082-261-3197

Printing company: Sanko, Inc 


\section{DISCLAIMER}

This report was prepared as an account of work sponsored by an agency of the United States Government. Neither the United States Government nor any agency thereof, nor any of their employees, make any warranty, express or implied, or assumes any legal liability or responsibility for the accuracy, completeness, or usefulness of any information, apparatus, product, or process disciosed, or represents that its use would not infringe privately owned rights. Reference herein to any specific commercial product, process, or service by trade name, trademark, manufacturer, or otherwise does not necessarily constitute or imply its endorsement, recommendation, or favoring by the United States Government or any agency thereof. The views and opinions of authors expressed herein do not necessarily state or reflect those of the United States Government or any agency thereof. 


\section{DISCLAIMER}

Portions of this document may be illegible in electronic image products. Images are produced from the best available original document. 


\section{Contents}

Introduction

A message from the chairman ........................................... i

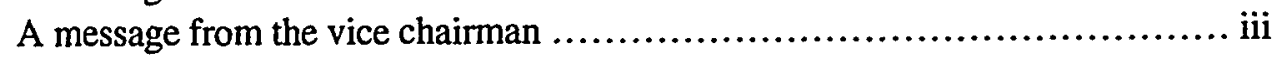

Fiscal Year 1995 Research Activities Report................................ 1

Active research protocols by RERF program ....................................... 2

Progress report by department .................................................... 11

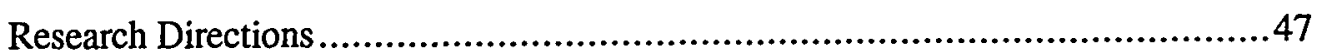

Major Meetings

22nd Scientific Council Meeting.......................................... 68

Blue Ribbon Review Committee Meeting ................................ 69

Abstracts of RERF Reports Published in Journals

RERF Reports..................................................... 71

Commentary and Review Series ..................................... 80

Publications in the Scientific Literature.................................. 82

Oral Presentations ..................................................... 87

Scientific Lectures and Seminars ....................................... 98

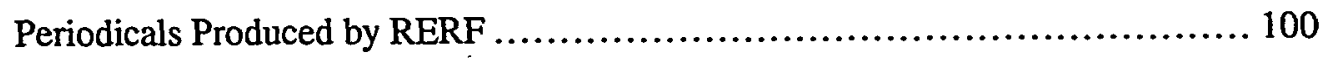

International Collaborative Activities .................................... 102

Ministry of Health and Welfare-entrusted Database Development ............... 109

20th Anniversary of the Establishment of RERF

Commemoration ceremony and lectures ............................................ 109

Open house......................................................................... 111

Visitors to RERF, Commendations, and In memoriam ............................ 112

Report from the Secretariat

29th Board of Directors Meeting...................................... 114 
Personnel ........................................................... 115

Organizational structure of RERF .................................... 117

Fiscal and property report........................................... 118

\section{Appendix}

29th Board of Directors Meeting

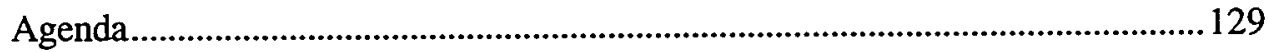

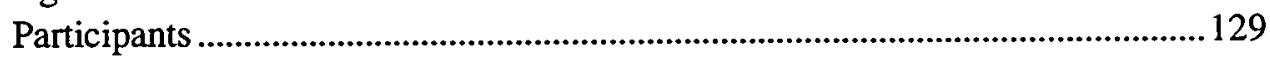

Minutes of the meeting...................................................................................131

22nd Scientific Council Meeting

Agenda.............................................................. 145

Participants...................................................... 147

General recommendations ............................................ 148

Detailed recommendations..................................................... 150

Directors, Supervisors, and Scientific Councilors ........................ 157

Consultants........................................................... 158

Expert Advisors...................................................... 161

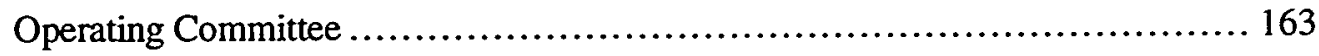

Standing Committees ................................................ 164

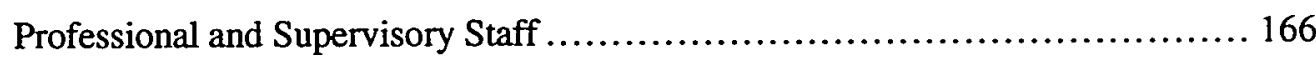

Summary Tables

Contacting Adult Health Study (AHS) participants and contacting families for permanent lymphocyte cell-line establishment .............. 173

Medical clinic visits .................................................. 173

Cryopreservation of blood samples obtained from AHS participants ......... 174

Tumor-registry accessions (medical records for malignant tumors only) ....... 175

Tissue-registry accessions ......................................... 175

Agreements on RERF International Collaborations........................ 176

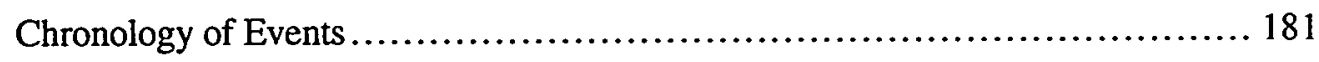




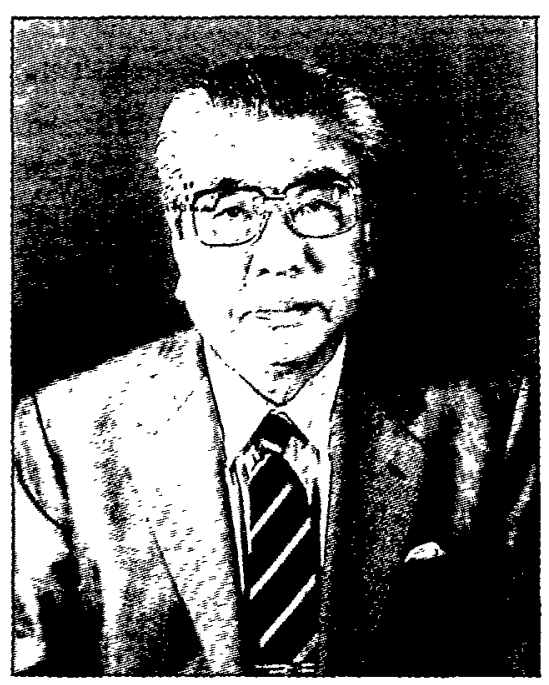

Itsuzo Shigematsu

\section{Introduction}

\section{A message from the chairman}

\section{by Itsuzo Shigematsu, MD}

On the occasion of the publication of the RERF annual report for fiscal year 1995, I wish to express my profound appreciation to the atomic bomb survivors and many other people concerned for their cooperation in the research program of the foundation.

This memorable year marks the 50th anniversary of the atomic bombing and the 20 th anniversary of the establishment of RERF, but the situation surrounding the foundation continues to be harsh. In addition to the problems of budget and personnel reduction owing to the stringent financial situation which has continued in the United States for two years, the US Department of Energy (DOE) indicated its intention to change the institution by entrusting the US administration of RERF to an institution other than the US National Academy of Sciences (NAS).

A budget reduction plan has been implemented since 1994. The Scientific Council, from the viewpoint of ensuring that the RERF research activities be maintained at the highest scientific level and that appropriate administration be maintained, recommended at the April 1995 Meeting, that views of a high-level international committee of distinguished scientists (Blue Ribbon Panel) should be sought concerning future RERF research activities. This recommendation was approved at the 29th Meeting of the Board of Directors, and the governments of the two countries were requested to take action for its materialization.

Following this, the Ministry of Health and Welfare and the DOE, in November 1995, appointed Professor Roger H. Clarke, Director, UK National Radiological Protection Board (NRPB), as Chairman of the Panel, and eight scientists representative of their respective disciplines, including radiological protection, epidemiology, radiation carcinogenesis and medical biology, as members.

The Panel held its first meeting at RERF in Hiroshima and Nagasaki from 4 to 8 February 1996, where the department chiefs reported on their research activities and earnest discussions were held on them. I believe the Panel members appreciated the depth of the scientific and medical knowledge gained from RERF research activities. The Panel will hold its second meeting at the NRPB in the United Kingdom in the suburbs of Oxford in May, and will submit the Panel's final report to the two governments in early July 1996. 
The pending issue of changing the institution to entrust the US administration of RERF was shelved until the Blue Ribbon Panel issues its conclusion. For the time being, the administration contract between DOE and NAS has been extended for two years.

This year marks the 10th anniversary of the Chernobyl nuclear power plant accident, and many commemorative events are scheduled to be held all over the world. Although RERF can be expected to be called upon for more active participation in them, the Chernobyl accident has given rise to a year-on-year increase of foreign researchers seeking to be trained, and there is an increasing demand worldwide for RERF to provide information. In response to these needs, the organization and functions of RERF should be reviewed for rationalization, and international cooperation should be further promoted.

To inform the general public more about our everyday research activities, we held our first open house at the Hiroshima Laboratory on 5 August. Nearly 900 visitors from all parts of the country including Hiroshima citizens came, and words of encouragement and various comments were received. This impressed upon the staff the importance of our future research activities.

RERF presently has numerous pending problems. Further efforts will be made for their early resolution so that the acquired research results may contribute ever more to the health and medical care of A-bomb survivors and largely to the world in the area of radiological protection.

Lastly, this year, as in past years, numerous A-bomb survivors have passed away. In behalf of RERF, I would like to express our condolences to the bereaved families and our hope that their souls may rest in peace. 


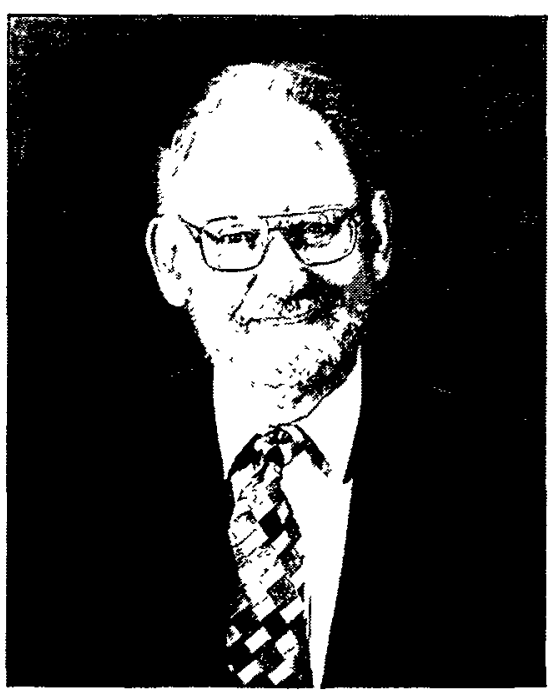

Seymour Abrahamson

\section{A message from the vice chairman}

\section{by Seymour Abrahamson, PhD}

This is my third term as a director and Chief of Research and my first term as Vice Chairman. I suspect that by the time this volume is published, I will have departed RERF. My arrival in August 1995 was to provide assistance to our program until such time as needed to allow a permanent director from the US to be recruited by the US National Academy. Much of my time has been devoted to helping develop material for the International Blue Ribbon Panel review of our research program, held in Hiroshima and Nagasaki during the first week of February. We await their final recommendation due by July. I wish to go on record as stating that our staff did a superb job in presenting their research program results and their future research plans. Three full days were clearly too short a time to cover the full range of our activities, but documents were sent to the Panel in advance that gave details of research in all departments, an historical overview, and future research objectives. Credit for the successful program must also be shared at every level of RERF staff, in every department.

Consciously or subconsciously, we are aware that any review panel can not help but be impressed with the quality and depth of our research program. Now that the pressure of these past months has eased, we have returned to our research obligations with a renewed sense of community spirit and purpose. Our goals continue to be to provide the world with the most important research on the long-term health effects of radiation.

The optimist in me says that both governments will now find the means to maintain RERF's unique research program in a fiscally responsible manner.

The optimist in me foresees a continuing research productivity in all areas: Critical information will accrue from the Life Span Study and Adult Health Study populations that will shape regulatory agencies' protection standards; genetic studies at the DNA level will provide the critical human data so urgently required-progress in this area is astounding; and our basic studies will contribute to unravelling the underlying mechanisms of cancer development. Because we can and will do these things, we will have fulfilled our obligations to the people of Hiroshima and Nagasaki for a safer world. 


\section{Fiscal Year 1995 Research Activities Report}

Fiscal Year 1995 has been an unusual year with respect to RERF's program. Beginning in October 1995, the activities of the office of the Chief of Research centered on preparing documents for the Blue Ribbon Panel meeting held in early February 1996 in both Hiroshima and Nagasaki. The drafts of these documents were sent to the entire Board for comments prior to their final distribution to the Panel. Much of the month preceding the meeting was devoted to reviewing the individual scientific presentations that were to be delivered by the staff at the Panel meeting. Their presentations were outstanding in clarity and importance to RERF's mission. Equally important to the success of the program was the collaboration of the Department Chiefs in preparing the documentation and organizing the meeting. In addition, the extraordinary effort provided by all the support services staff should be recognized.

Stability of funding and of staffing are critical issues that directly impact RERF's research productivity. Negotiations between our funding agencies suggest that stability may be achieved relatively soon. This is very much to be desired. Chief among our major concerns for the future of the program is maintaining a strong scientific staff. We have lost three researchers in 1995, including one department chief, and by the end of next year, another department chief will retire. As we stated in the future planning document, the highest priority must be given to adding epidemiologists to our staff. Obviously the epidemiology program is the focal point of our program. Surprisingly, however, it has the smallest scientist staff size of all departments. It is critical that we attract well trained Japanese, US or other epidemiologists as soon as possible. I also fear the loss of at least one or both of our US computer staff members will critically hamper data analysis in all areas of RERF. Recruitment of a new staff member in this area has been unsuccessful.

The issue of staffing stability relates not only to attracting excellent candidates to fill vacated positions, but also to maintaining a research climate that provides our existing staff with an incentive to continue working here. The US staff, small in size, is nevertheless essential to the continued productivity of the program. They have an average of 8-year working time at RERF. The destabilizing issues of the past 18 months have taken their toll particularly on this group both in directly observable ways, such as job uncertainty, and in less obvious ways, primarily the restructuring of US fringe benefits previously available. These factors have occurred almost simultaneously and, I believe, will shortly lead to a loss of key personnel. Key personnel are of course replaceable, but the learning curve is slow for this new environment and the adaptation to a new culture creates hardships not readily overcome by all new staff members. Nor can adequate replacements be obtained from staffing with post-doctoral fellows on 2-year rotations. 
Simply put, we need to encourage, not discourage, a small but vital permanent contingent of US staff.

The Chief of Research positionship has been staffed by a US director since the inception of RERF, but the position has been for the most part a "revolving door" in which the average tenure has been 2 years; hardly enough time to learn the program, the people and the job responsibilities. Some way should be found to keep an effective person for a longer term or reconsider whether the position could not be better staffed by an existing Department Chief of proven abilities from either country.

\section{Active research protocols by RERF program}

\section{April 1995-31 March 1996}

Editor's note: Manuscripts (司) as yet unpublished in scientific journals are listed under the relevant research protocols. These have been approved by the RERF directors for journal publication. Reports that have been published in scientific journals are listed with their summaries beginning on page 71.

\section{Life Span Study}

RP 2-61 Study of mortality in children exposed in utero [Editor's note: See ABCC Technical Report 21-60 for the full text.]

1-75 Research plan for RERF study of life-span of A-bomb survivors, Hiroshima and Nagasaki [Editor's note: For the full text of this platform protocol, see ABCC Technical Report 4-59.]

濖 Vaeth M, Pierce DA, Preston DL: The impact of neutron radiation on cancer mortality among the A-bomb survivors

Pierce DA, Shimizu Y, Preston DL, Vaeth M, Mabuchi K: Studies of the mortality of atomic bomb survivors. Report 12, Part I. Cancer: 1950-1990

6-88 Comparative analysis of the LSS population and a cohort of 265,000 Japanese men and women

\section{Adult Health Study}

RP 2-75 Research plan for RERF Adult Health Study, Hiroshima and Nagasaki [Editor's note: This is a platform protocol.]

惯 Yamada M, Wong FL, Kodama K, Sasaki H, Shimaoka K: Longitudinal trends in total cholesterol levels in a Japanese cohort, 1958-1986 


\section{Immunology}

RP 36-63 Blood groups in Adult Health Study and in utero ATB subjects Hiroshima and Nagasaki

3-87 Cellular immune function and its relationship to in vitro $\mathrm{T}$ lymphocyte radiosensitivity and $\mathrm{MN}$ blood group locus mutation frequency in A-bomb survivors: Precursor frequency analysis of mitogen- and antigen-responsive blood lymphocytes

Iwamoto KS, Mizuno T, Ito $\mathrm{T}$, Tsuyama $\mathrm{N}$, Kyoizumi S, Seyama $\mathrm{T}$ : Gain-of-function p53 mutations enhance alteration of the T-cell receptor following X-irradiation, independently of the cell cycle and cell survival

㬝 Ishioka N, Kyoizumi S, Umeki S, Hirai Y, Akiyama M, Kodama T, Ohama $\mathrm{K}$ : Stimulated rapid expression in vitro for early detection of in vivo T-cell receptor mutations induced by radiation exposure

7-87 X-ray radiosensitivity of lymphocytes in vitro from A-bomb survivors. Part 3: Transformation of B cells by Epstein-Barr virus and their cryopreservation (addendum to RP 3-86)

7-88 Study of somatic mutations at the glycophorin A locus in erythrocytes of atomic bomb survivors

圈 Kyoizumi S, Akiyama M, Cologne JB, Tanabe K, Nakamura N, Awa AA, Hirai Y, Kusunoki Y, Umeki S: Somatic cell mutations at the glycophorin A locus in erythrocytes of atomic bomb survivors: implications for radiation carcinogenesis

7-89 Screening of stem cell mutation in lymphoid lineage among A-bomb survivors and its characterization

9-89 Detecting erythrocyte mutations at the glycophorin A locus in Nagasaki A-bomb survivors and in Hiroshima area poison gas workers (addendum to RP 7-88)

Kyoizumi S, Akiyama M, Cologne JB, Tanabe K, Nakamura N, Awa AA, Hirai Y, Kusunoki Y, Umeki S: Somatic cell mutations at the glycophorin A locus in erythrocytes of atomic bomb survivors: implications for radiation carcinogenesis

11-89 A pilot study for detecting somatic mutations at the HLA-A locus in lymphocytes

2-90 Cryopreservation of blood cells from Hiroshima and Nagasaki Adult Health Study participants 
RP 4-90 Establishment of a method for HLA-DQ and DP gene typing using

(Reactivated) the polymerase chain reaction

1-93 Repertoire of T-cell antigen receptors and activity of hematopoietic progenitor cells in peripheral blood of atomic-bomb survivors (addendum to RPs 3-87, 4-87 and 7-89)

2-93 Development of assay for somatic mutation at the locus of the neutrophil Fc $\gamma$ receptor III gene and preliminary study of atomicbomb survivors

\section{Special clinical studies}

RP 4-85 Incidence and risk factors of coronary heart disease (CHD) in Japanese men living in Japan and Hawaii, 1966-78 (addendum to Research Plan TR 12-71)

6-85 Study of M-proteinemia in the Adult Health Study sample (addendum to RP 9-79)

4-86 Evaluation of index of physiological measurements: A predictor of mortality or morbidity associated with aging

5-86 Dietary habit survey using a simple and computerized diet survey system (addendum to RP 8-83)

11-86 Prevalence of hyperparathyroidism in atomic bomb survivors during AHS cycle 15, Hiroshima and Nagasaki

5-87 Radiation-related damage to the developing human brain

2-89 Hypercalcemia in A-bomb survivors, Hiroshima and Nagasaki (addendum to RP 11-86)

3-89 Osteoporosis in Hiroshima atomic bomb survivors

3-90 The association of serum cholesterol with noncardiovascular mortality and morbidity in the Adult Health Study population

3-91 A comparative study of vertebral fracture prevalence among Japanese, Japanese-Americans in Hawaii, and Caucasians in Minnesota

䀏 Fujiwara S, Huang C, Ross PD, Yamada M, Kodama K, Davis JW, Wasnich RD: Osteoporosis risk factor profiles differ between native Japanese and Japanese-Americans 
Huang C, Ross PD, Fujiwara S, Davis JW, Epstein RS, Kodama K, Wasnich RD: Determinants of vertebral fracture prevalence among native Japanese women and women of Japanese descent living in Hawaii

RP 5-92 Study of senile dementia among the Adult Health Study subjects

6-92 Establishment and operation of a system for collecting and storing leukemia cells

9-92 Study of liver diseases in the Adult Health Study sample: Relationship between radiation dose and infection by $\mathrm{B}$ and $\mathrm{C}$ hepatitis virus

4-93 Epidemiologic study of antiadult T-cell leukemia-associated antigen antibodies in the Adult Health Study sample, Nagasaki (addendum to RP 1-85)

Oishi K, Shibata Y, Nakamura T, Tsujihata M, Akahoshi M, Matsuo T, Tomonaga $\mathrm{M}$, Nagataki S, Shimaoka $\mathrm{K}$ : Autoantibodies and immunoglobulins in atomic bomb survivors with human T-lymphotropic virus type I

5-93 Longitudinal study of hormone indicators of menopause in perimenopausal female atomic-bomb survivors

1-95 Effects of menopause on risk factors for ischemic heart disease: A longitudinal study of the Nagasaki Adult Health Study sample

\section{Histopathology}

RP 5-89 Pathology studies in Hiroshima and Nagasaki. Revised research plan [Editor's note: Formerly RP 3-75, a platform protocol.]

8-89 Senile changes of the brain in Hiroshima and Nagasaki A-bomb survivors

\section{Cell biology}

RP 18-81 Pathophysiology and radiation response of human thyroid cells in culture and in grafts in athymic nu/nu mice

7-92 Molecular analysis of the p53 tumor-suppressor gene in breast cancers of atomic-bomb survivors (with addendum)

3-93 Molecular analysis of skin cancer in atomic-bomb survivors 
占 Iwamoto KS, Mizuno T, Ito T, Akiyama M, Takeichi N, Mabuchi K, Seyama T: Feasibility of using decades-old archival tissues in molecular oncology/epidemiology

RP 7-93 Molecular analysis of thyroid cancers among atomic-bomb survivors

2-94 Molecular analysis of hepatocellular carcinoma among atomic-bomb survivors

圈 Iwamoto KS, Mizuno T, Ito T, Akiyama M, Takeichi N, Mabuchi K, Seyama T: Feasibility of using decades-old archival tissues in molecular oncology/epidemiology

2-95 Pilot study: Characterization of monoclonal gammopathy by studying the role of the BSAP gene in CD19 antigen expression

\section{Biochemical genetics}

RP 5-85 Culture of permanent lymphocyte cell lines as sources of biological samples for investigation of genetic effects of radiation on children of atomic bomb survivors

7-85 Study to develop methods of DNA analysis for detection of mutations in children of atomic bomb survivors

\section{Cytogenetics}

RP 8-93 Cytogenetic study in the Adult Health Study population by fluorescence in situ hybridization (FISH)

\section{$F_{1}$ studies}

RP 4-75 Research plan for RERF studies of the potential genetic effects of atomic radiation; Hiroshima and Nagasaki. Part 1. Mortality study of children of atomic bomb survivors [Editor's note: This is a platform protocol.]

䁇 Akahoshi M, Soda M, Carter RL, Nakashima E, Shimaoka K, Seto S, Yano $\mathrm{K}$ : Correlation between systolic blood pressure and physical development in adolescence

\section{Special cancer studies}

RP 29-60 Detection of leukemia and related disorders [Editor's note: See ABCC Technical Report 5-65 for the full text.] 
RP 7-76 The value of Adult Health Study family history records in the determination of genetic influences on the development of cancer and other disorders

14-79 Interaction between radiation dose and host factors. An epidemiological case-control study of female breast cancer in atomic bomb survivors

8-85 Incidence study on malignant and benign genital tumors among females, Hiroshima and Nagasaki, 1950-80

2-86 Collection of surgically removed cancer tissues from A-bomb survivors: Special reference to thyroid and breast cancers

6-86 Ultrasonographic screening of Adult Health Study participants to detect cancer and other diseases

9-88 Guidelines for the conduct of site-specific cancer incidence studies among A-bomb survivors, Hiroshima and Nagasaki

5-90 Primary liver cancer incidence study among atomic-bomb survivors, 1958-87

1-91 Studies on salivary gland tumors among the RERF Extended Life Span Study cohort, Hiroshima and Nagasaki, 1950-87

氨 Saku T, Hayashi Y, Takahara O, Matsuura H, Tokunaga M, Tokuoka S, Soda M, Land CE, Mabuchi K: Salivary gland tumors among atomic bomb survivors, $1950-87$

2-91 Studies on skin cancer incidence among the RERF Extended Life Span Study cohort, Hiroshima and Nagasaki, 1950-87

6-91 Studies on thyroid tumor incidence among the RERF Extended Life Span Study cohort, 1950-87

2-92 Studies on ovarian tumor incidence among the RERF Extended Life Span Study cohort, 1950-87

4-92 Incidence study of tumors of the central nervous system among atomic-bomb survivors

6-93 Breast-cancer incidence among atomic-bomb survivors, 1950-90 (supersedes RP 1-90)

1-94 Studies on lung-cancer incidence among the atomic-bomb survivors, 1950-90 
RP 3-94 Incidence of lymphoid malignancies among the atomic-bomb survivors, 1950-90

\section{A-bomb dosimetry studies}

RP 18-59 Shielding survey and dosimetry study [Editor's note: See ABCC Technical Report 7-67 for the full text.]

圈 Vaeth M, Pierce DA, Preston DL: The impact of neutron radiation on cancer mortality among the A-bomb survivors

娄 Otake M, Neriishi K, Schull WJ: Cataract in atomic bomb survivors based on a threshold model and the occurrence of severe epilation

10.86 Radiation dose estimates using tooth samples. Part 1. Collection of tooth samples from A-bomb exposed people in Hiroshima and Nagasaki

1-92 Radiation dose estimates using tooth samples. Part 2. Use of electron spin resonance on tooth enamel from Hiroshima atomic bomb survivors

\section{Medical dosimetry studies}

RP 7-86 Doses to Adult Health Study participants from RERF radiological examinations, Hiroshima and Nagasaki

8-86 Ionizing radiation for medical reasons reported by Adult Health Study participants, Hiroshima and Nagasaki

8-87 Organ doses from medical X-ray exposures (addendum to RP 8-84)

5-91 Radiation-therapy-related cancer among Life Span Study subjects (addendum to RP 7-81)

\section{Tumor registry and tissue registry}

RP 18-61 Tumor registry study in Hiroshima and Nagasaki [Editor's note: See ABCC Technical Report 2-61 for the full text.]

Following are tissue registry-related protocols that are also listed under the category Special Cancer Studies.

RP 29-60 Detection of leukemia and related disorders [Editor's note: See ABCC Technical Report 5-65 for the full text.] 
RP 9-88 Guidelines for the conduct of site-specific cancer incidence studies among A-bomb survivors, Hiroshima and Nagasaki

5-90 Primary liver cancer incidence study among atomic bomb survivors, 1958-87

1-91 Studies of salivary gland tumors among the RERF Extended Life Span Study cohort, Hiroshima and Nagasaki, 1950-87

曾 Saku T, Hayashi Y, Takahara O, Matsuura H, Tokunaga M, Tokuoka S, Soda M, Land CE, Mabuchi K: Salivary gland tumors among atomic bomb survivors, $1950-87$

曾 Land CE, Saku T, Hayashi Y, Takahara O, Matsuura H, Tokuoka S, Tokunaga $\mathrm{M}$, Mabuchi $\mathrm{K}$ : Incidence of salivary gland tumors among atomic bomb survivors, 1950-1987. Evaluation of radiation-related risk

2-91 Studies on skin cancer incidence among the RERF Extended Life Span Study cohort, Hiroshima and Nagasaki, 1950-87

6-91 Studies on thyroid tumor incidence among the RERF Extended Life Span Study cohort, 1950-87

2-92 Studies on ovarian tumor incidence among the RERF Extended Life Span Study cohort, 1950-87

4-92 Incidence study of tumors of the central nervous system among atomic-bomb survivors

6-93 Breast-cancer incidence among atomic-bomb survivors, 1950-90 (supersedes RP 1-90)

1-94 Studies on lung-cancer incidence among the atomic-bomb survivors, 1950-90

3-94 Incidence of lymphoid malignancies among the atomic-bomb survivors, $1950-90$

\section{List of research protocols inactive during FY1995*}

RP 15-81 Case-control study of lung cancer among atomic bomb survivors (Special cancer studies)

*Program category is shown in parentheses at the end of each RP title. 
RP 6-89 Incidence of radiation-related skin lesions in the Adult Health Study populations of Hiroshima and Nagasaki, 1958-89 (Special clinical studies)

\section{List of research protocols terminated in FY1995*}

RP 2-85 Cytogenetic and pathological studies of normal and heat burn cicatricial skin fibroblasts in atomic bomb survivors (Cytogenetics)

濖 Honda T, Sadamori N, Oshimura M, Horikawa I, Omura H, Komatsu K, Watanabe M: Spontaneous immortalization of cultured skin fibroblasts obtained from a high-dose atomic bomb survivor

1-89 Prevalence of radiation-related skin lesions in the Adult Health Study population, Hiroshima and Nagasaki (Special clinical studies)

湩 Yamada M, Kodama K, Fujita S, Akahoshi M, Yamada S, Hirose R, Hori M: Prevalence of skin neoplasms among the atomic bomb survivors

7-91 Analysis of radiation sensitivity mechanism in the severe combined immunodeficient (SCID) mouse (Cell biology)

圈 Araki R, Itoh $\mathrm{M}$, Hamatani $\mathrm{K}$, Abe $\mathrm{M}$ : Normal $\mathrm{D}-\mathrm{J}_{\mathrm{H}}$ rearranged products of IgH gene in SCID mouse bone marrow

䕊 Hamatani K, Itoh M, Watanabe F, Teraoka H, Araki R, Abe M: Importin$\beta$ and $3^{\circ}$ untranslated region of its gene complement the high radiosensitivity and abnormal V(D)J recombination of SCID mice

氨 Araki R, Kaku M, Itoh M, Hamatani K, Usui T, Abe M: Recombination activating gene (RAG)-1 and 2 encoding proteins expressed by the baculovirus system

\section{List of manuscripts based on research protocols other than ones active in FY1995}

RP 24-62 Growth and development studies, Hiroshima and Nagasaki [Editor's note: see ABCC Technical Report 5-62 for the full text.]

图 Otake M, Schull WJ: Threshold of radiation-related severe mental retardation in prenatally exposed survivors: a reanalysis

16-78 Ophthalmologic changes related to radiation exposure and age in the Adult Health Study sample, Hiroshima and Nagasaki

\footnotetext{
*Program category is shown in parentheses at the end of each RP title.
} 
Otake M, Neriishi K, Schull WJ: Cataract in atomic bomb survivors based on a threshold model and the occurrence of severe epilation

RP 5-88 The relationship between age at menopause and A-bomb exposure in Adult Health Study participants

Akahoshi M, Soda M, Nakashima E, Shimaoka K, Seto S, Yano K: Effects of menopause on trends of serum cholesterol, blood pressure, and body mass index

\section{Progress report by department Department of Epidemiology}

RP 3-94 Incidence of lymphoid malignancies among the atomic-bomb survivors, 1950-90. K Nanba, T Matsuo, M Tokunaga, T Jubashi, Y Fujita, M Soda, H Dohy, N Kamada, M Tomonaga, LB Travis, DL Preston, K Mabuchi, CE Land, S Tokuoka.

The relationship between lymphopoietic malignancies and radiation exposure is complicated because of evident variability by diagnostic category and uncertainty about potential for radiation carcinogenesis for certain important categories. Thus, the risk for acute lymphocytic leukemia was markedly elevated among the atomic-bomb survivors during the early years, particularly among children, while there is no convincing evidence of a radiation-induced excess of chronic lymphocytic leukemia. Multiple myeloma, derived from terminally differentiated B cells, has been identified as a radiation-induced cancer in the analysis of the LSS mortality data, but recently published incidence data demonstrated a weak association with radiation exposure. Studies of non-Hodgkin's lymphoma in the LSS have also demonstrated variable and inconsistent results, at least in part because of different sources of diagnostic data used and diagnostic variability. In contrast, recent advances in immunological studies of malignant lymphoma are remarkable. Surface markers applied to tumor cells can be used to distinguish $\mathrm{B}$ and $\mathrm{T}$ cells involved in malignant lymphomas, and a new classification has replaced the conventional classification of lymphomas. The objective of the present study is to investigate all lymphopoietic tumors (lymphomas, multiple myeloma, and lymphocytic leukemias) between 1950 and 1990 in the LSS. Emphasis will be on confirming and classifying cases using modern techniques and classification applied to cases ascertained from all sources. The study will involve both hematologists and pathologists engaged in lymphoid tumor research. Non-Hodgkin's lymphomas will be classified by immunohistochemical studies into $\mathrm{T}$ - or B-cell lymphomas, and diagnosis of adult T-cell leukemia will be based on detection of proviral DNA of HTLV-I using archived tissues. This study has been in the first phase of investigation, ie, screening of several thousands of potential cases with a wide range of diagnoses to which lymphoid tumors may be misclassified. At least another year will 
be required to complete the first phase and a pathology review. Data analyses will begin in 2 or 3 years.

RP 1-94 Studies on lung-cancer incidence among the atomic-bomb survivors, 1950-90. H Egawa, $T$ Matsuo, S Yenehara, Y Fujita, E Nakashima, M Soda, $M$ Tokunaga, S Tokuoka, S Akiba, K Mabuchi, DL Preston, CE Land.

Lung cancer is a well established late effect of radiation exposure in various irradiated populations including the atomic-bomb survivors. However, there remain several specific issues and questions. These include the specificity of various cell types involved in radiation- versus smoking-related cancers, confounding and joint effects of smoking in relation to radiation exposure, and delineation of temporal trends with allowance given to the age-at-exposure and attained-age effects. ICRP also has recently published a new report on lung cancer risk from inhaled radionuclides modeled on an anatomical basis in terms of lung "compartments." New information on anatomical distribution of lung cancers resulting from uniformly distributed radiations may be useful for evaluating the ICRP model. This RP was developed to address these questions and issues. The study will evaluate lung cancer incidence in the Life Span Study for the period of 1950 to 1990 . Pathology reviews have been started and will continue during the next 12 months. Data analyses will begin next year and first draft papers will be prepared in the following year. The study will be completed in the next 3 to 4 years.

RP 5-93 Longitudinal study of hormone indicators of menopause in perimenopausal female atomic-bomb survivors. T Rose, M Soda, M Yamada, H Sasaki, M Akahoshi, JB Cologne, K Kodama.

A recent analysis of self-reported menopause data available for the Nagasaki portion of the Adult Health Study sample showed increased incidence of early onset of menopause associated with radiation exposure from the atomic bombs. This RP was developed to measure biochemical indicators to estimate the incidence of early menopause in a subset of the AHS sample in Hiroshima and Nagasaki. Over a 4-year period, occurrence of early menopause is monitored by measuring levels of two perimenopausal hormones, follicular stimulating hormone (FSH) and estradiol (E2), at a 6-month interval among female AHS subjects who were born between 1940 and 1945 and who are under 50 years of age at the initial baseline examination. A total of 211 women have been enrolled in this study since the baseline examination which started in early 1994. Following the baseline, a fasting blood sample is also taken at each 6-month visit, and information on drinking habits and any changes in menopausal symptoms are documented. A quality control study is also undertaken on 10 to 20 women to evaluate the sources of variability in hormone assays. As of this writing, the 
subjects have received three follow-up examinations after the baseline. This study is expected to continue till 1998.

RP 6-93 Breast cancer incidence study among atomic-bomb survivors, 195090. M Tokunaga, CE Land, S Tokuoka, T Ikeda, M Soda, K Mabuchi.

This is the latest of a series of breast cancer incidence surveys conducted by Tokunaga and Land. A previously completed fourth survey (RP 1-90) included 807 primary breast cancer cases and 20 secondary breast cancer cases diagnosed from 1950 to 1985. A strong linear radiation-dose response was found, with the highest excess relative risk averaged over the entire observation period occurring among women who were $<20$ years of age at the time of exposure to radiation from the bombings. A much lower, marginally significant dose response was seen among women exposed at $>40$ years. A strikingly high risk per unit of radiation for early-onset breast cancer cases led the authors to suspect the possible presence of a genetically susceptible subgroup. A new research protocol expands the breast cancer series through 1990. Data collection for this series has been completed, adding 261 newly accessed cases (250 for the period of 1986 to 1990 and 11 prior to 1986). Of these, 58 cases were exposed at $<10$ years, and this should strengthen risk estimates for this age-at-exposure group. Data analysis has begun. It is expected that the analyses will be completed within the next 12 months, resulting in publication of the new incidence data. Upon completion of this RP, a new RP will be proposed within 2 to 3 years to further update the series, possibly adding another 5 years of follow-up.

RP 4-92 Incidence study of tumors of the central nervous system among atomic-bomb survivors. $S$ Yenehara, $H$ Fujii, $M$ Kishikawa, $T$ Kobuke, DE Thompson, M Soda, M Tokunaga, S Tokuoka, K Mabuchi, E Ron, DL Preston, CE Land.

Tumors of the nervous system have been associated with late effects of medical radiation at all ages, although detailed risk assessment has been difficult primarily due to small numbers of cases. Until recently, there has been very limited information on brain and other tumors of the nervous system in the LSS cohort. In the recent solid cancer incidence report, a suggestive dose response was found for tumors of the nervous system (except for the brain) among those exposed at ages $<20$ years. These findings prompted the present study. The objective is to ascertain malignant and benign tumors of the central nervous system in the LSS during the period 1950 to 1987 under the guidelines established for conducting site-specific studies. All the work related to case finding and histological review has been completed, and a total of 374 tumors, benign and malignant, has been identified. Of these, $282(75 \%)$ are histologically confirmed. Menigioma is most frequent $(41 \%)$, followed by schwannoma (or neurilemmoma) of the cranial and spinal nerves (25\%), cerebral and 
spinal glioma (19\%) and pituitary adenoma (11\%). Preliminary analysis shows a significant dose response for neurilemmoma. All analyses will be completed within the next 12 months, and the writing of papers will begin. It is expected that the study will be completed within 2 to 3 years.

RP 2-92 Studies on ovarian tumor incidence among the RERF Extended Life Span Study cohort, 1950-87. S Tokuoka, K Kawai, K Inai, Y Shimizu, E Nakashima, M Tokunaga, M Soda, K Mabuchi, CE Land.

An increased risk of ovarian cancer among the atomic-bomb survivors has been reported from an earlier site-specific study of ovarian cancer as well as the analyses of LSS mortality and incidence. No clear evidence has been available for any specific histologic type being particularly associated with radiation exposure. In the previous study, the frequency of benign tumors of the ovary detected at autopsy was also found to increase with radiation dose. The present study extends the previous ovarian cancer series by 7 years (through 1987) and also includes a systematic ascertainment of benign tumors. All work related to case finding and histological verification has been completed. A total of 727 ovarian tumors have been identified, of which 263 are malignant and 464 are benign, and 218 (83\%) of the malignant tumors and 442 (95\%) of the benign tumors have been histologically verified. Data analysis has started and will continue during the next 12 months. It is expected that the study will be completed in 2 to 3 years with publication of papers.

RP 6-91 Studies on thyroid tumor incidence among the RERF Extended Life Span Study cohort, Hiroshima and Nagasaki, 1950-87. Y Hayashi, B Tsuda, S Akiba, S Fujita, M Tokunaga, S Tokuoka, E Ron, CE Land, K Mabuchi.

Thyroid cancer was one of earliest solid cancers found to be increased in atomicbomb survivors. Several thyroid cancer studies have been conducted among the atomic bomb survivors over the last 40 years. The aim of the present investigation is to update and expand the earlier thyroid cancer incidence series (through 1979), including both benign and malignant tumor cases diagnosed between 1950 and 1987. An increased number of cases over an extended study period should allow more detailed risk analyses. All the work related to case ascertainment and histological grouping has been completed. A total of 2637 potential cases were reviewed, resulting in the identification of a total of 915 thyroid tumor cases. Histological verification was possible for 853 (93\%) of these cases: 560 with malignant tumors, 292 with benign tumors and one with intrathyroid parathyroid lesion. The majority of malignant thyroid carcinomas are of the papillary type (96\%), followed by follicular type (1\%), malignant lymphoma $(1 \%)$, medullary $(<1 \%)$ and undifferentiated carcinoma $(<1 \%)$. The 539 papillary carcinomas include 298 microcarcinomas detected mostly at autopsy. Data are currently being entered into a database. Data analysis is expected to begin shortly 
and will continue during the next 12 months. Papers will be written and submitted for publication in the next 2 to 3 years.

RP 2-91 Studies of skin cancer incidence among the RERF Extended Life Span Study cohort, Hiroshima and Nagasaki, 1950-87. M Kishikawa, T Kobuke, M Iseki, N Sadamori, S Yamamoto, M Soda, M Tokunaga, S Tokuoka, K Mabuchi, E Ron, DL Preston, CE Land.

An elevated risk of skin cancer, mostly basal cell carcinoma, has been reported from the follow-up of children treated with radiation for tinea capitis, enlarged thymus, and various head and neck diseases. The LSS solid cancer incidence report demonstrated, for the first time, an increased risk of skin cancer in the atomic-bomb survivors. Since the completeness of ascertainment of skin cancer from tumor registries is questionable and also since diagnoses of skin cancer are highly variable among physicians and pathologists, the present study is designed to provide extended case finding and a standardized pathology review for diagnosis and histological typing of skin tumors. All work related to case ascertainment has been completed, and analyses have almost been completed. The total number of skin cancer cases obtained for the study period (1950-1987) was 343, of which 301 (88\%) were histologically verified by a panel of pathologists. Basal cell carcinoma comprised $37 \%$ of the cases; squamous cell carcinoma, 33\%; Bowen's disease, 14\%; malignant melanoma, 5\%; and other various types, $11 \%$. A significant dose response was demonstrated for non-melanoma skin cancer as a group, and especially for basal cell carcinoma and other epithelial skin cancers (non-basal, non-squamous cell). There was no evidence of a dose response for squamous cell carcinoma. Noteworthy was the absence of a suspected combined effect of UV and ionizing radiation exposure and a strong effect of age at exposure on basal cell carcinoma. Two draft papers have been written. The first paper presents histological characteristics of skin cancer; the second paper discusses risk estimates. These papers will be submitted for internal review within the next 12 months and for journal publication thereafter. With the publication of the papers, this study is expected to be completed.

RP 1-91 Studies of salivary gland tumors among the RERF Extended Life Span Study cohort, Hiroshima and Nagasaki, 1950-87. T Saku, Y Hayashi, O Takahara, M Tokunaga, S Tokuoka, K Mabuchi, M Soda, E Ron, DL Preston, CE Land.

Despite much evidence in the literature linking increased risk of benign and malignant tumors of the salivary glands to ionizing radiation exposure, little detail is known about the risk beyond linear dose response because these tumors are rare. Also, minor salivary gland tumors are not identifiable under the conventional coding scheme (International Classification of Diseases) used by tumor registries. The present study are designed to include both benign and malignant tumors of the major and minor 
salivary glands diagnosed between 1950 and 1987. A large number of potential cases are screened including those diagnoses under which salivary gland tumors are likely to be misclassified and coded. Pathology reviews are conducted in a standardized manner. This study has almost been completed. The total number of cases identified for the above period is 135 ( 41 malignant and 94 benign tumor cases). Analysis shows a significant dose response for both benign and malignant tumors. Most of the dose response for malignant tumors is provided by an exceptionally strong dose response for a particular type, mucoepidermoid carcinoma, and most or all of the dose response is attributable to that for Warthin's tumor. Two papers have been submitted for journal publication. The first paper presents methods used for case-ascertainment and histopathological features of salivary tumors. The second paper presents results of risk assessment. This RP will be terminated with publication of the two papers, hopefully within the next 12 months.

RP 5-90 Primary liver cancer incidence study among atomic-bomb survivors, Hiroshima and Nagasaki, 1958-1987. T Fukuhara, E Itakura, M Yamamoto, S Tokuoka, K Mabuchi, T Seyama, N Nakamura, M Akiyama, JB Cologne, M Soda, M Tokunaga, GW Beebe.

Liver cancer among the atomic-bomb survivors is of special interest because: (1) it is a major site of cancer in Japan and has been shown to respond not only to radiation by alpha particles but also to low-LET radiation by the recently published LSS cancer incidence data; (2) roles of hepatitis virus infection in liver cancer are rapidly being understood; and (3) an earlier RERF study showed increased prevalence of hepatitisB surface antigen (HBsAg) positivity in exposed individuals. Since diagnostic misclassification is a major concern for liver cancer, the primary objective of this study is to assess the relationship between atomic bomb radiation and liver cancer based on data confirmed by a panel of pathologists. The pathology review will include not only tissue slides but pathology, clinical and other diagnostic information. Another objective is to investigate, in a nested case-control study, the possible role of $\mathrm{HBV}$, and possibly $\mathrm{HCV}$, infection in radiation-related liver cancer. In the case-control study, two matched controls are selected for each autopsied liver cancer case. The pathology review extends from histologic classification of liver cancer in the incidence series to diagnosis of any accompanying liver cirrhosis and testing for HBV markers in the case-control study. In a companion study conducted in the Department of Radiobiology, molecular techniques are also used to characterize the $\mathrm{HB}$ and $\mathrm{HC}$ viruses. Case ascertainment and diagnostic confirmation have been completed, resulting in identification of 864 cases of primary liver cancer for the study period 1958 to 1987 . Of these, $42 \%$ were histologically verified. A majority (84\%) of liver cancers were hepatocellular carcinomas, $74 \%$ of which were accompanied by liver cirrhosis. The Radiobiology Department has developed techniques for assaying HCVs using 
tissue samples, and work is underway to study HCVs in the case-control study. The analysis of the incidence data and the HBV data will be completed within a year and preparation of papers will begin. It is also expected that HCV data will become available within a year. Analysis including both HBV and HCV data will then begin. The study will be completed within the next three years.

\section{RP 5-89 Pathology studies in Hiroshima and Nagasaki. Revised research plan (Formerly RP 3-75, a platform protocol). S Tokuoka, M Tokunaga, K Mabuchi.}

The Pathology Program, which was started at the inception of $A B C C$, has undergone several major revisions. In the beginning, it consisted almost entirely of an autopsy program in the cities of Hiroshima and Nagasaki with emphasis on the procurement of "exposed" individuals. In 1961, the pathology population was defined as a subset of the LSS sample in order to include adequate controls. An active and aggressive autopsy procurement effort was introduced, which resulted in increased autopsy rates. The autopsy rates reached a peak in the range of $40 \%-45 \%$ in the early 1960 s, but declined subsequently. In 1979, active solicitation of autopsies was terminated in favor of the completely voluntary cooperation of local physicians. In 1987, the program was redesigned to place major emphasis on patho-epidemiological follow-up studies of the atomic bomb survivors in search for specific histologic, cytologic or other tissue changes directly or indirectly attributable to irradiation. The present RP reflects the latest change. Under newly developed guidelines, "Guidelines for the site-specific cancer incidence studies" (RP 9-88), several site-specific studies have been initiated and are currently in progress. The autopsy program at RERF was completely terminated in 1988. However, through the ABCC/RERF Pathology Program, a large number of LSS autopsies (about 7500 cases) and surgical tissue samples (about 13,000 cases) have been collected over the years. These archived tissues have been found to be useful for molecular studies. However, for recently diagnosed cases, we must seek tissue samples from outside hospitals. Tissues used for a series of site-specific studies and molecular studies at RERF are obtained under an agreement signed for the Pathology Program. For future research in this field, it is essential that we establish a mechanism to identify and secure important tissue samples, especially from outside RERF. Within the next 12 months, a concentrated effort should be made to develop a community-wide agreement to create such a tissue storage program. Detailed plans of each site-specific and molecular study underway are described under specific RPs. 
RP 9-88 Guidelines for the conduct of site-specific cancer incidence studies among A-bomb survivors, Hiroshima and Nagasaki. M Tokunaga, K Mabuchi, S Tokuoka, CE Land.

The intent of the guidelines described in this RP is to simplify the preparation of research plans for site-specific cancer incidence studies and to provide uniformity in basic design and operation of studies. The guidelines state that case ascertainment be undertaken through the Hiroshima and Nagasaki tumor registries supplemented by an extended case-finding search. Histopathological verification should be achieved by a panel of collaborating pathologists specializing in the respective areas. Tumor types are to be classified using internationally accepted tumor classification systems. Analyses of data should be performed by RERF and/or other participating epidemiologists and statisticians. Under these guidelines, the following site-specific cancer incidence studies are currently undertaken: liver (RP 5-90); salivary glands (RP 1-91); skin (RP 2-91); thyroid (RP 6-91); ovary (RP 2-92); central nervous system (RP 4-92), breast (RP 6-93), lung (RP 1-94) and lymphoid tissues (RP 3-94). Work on case ascertainment and pathology review has been completed for breast cancer, ovary cancer, liver cancer, and central nervous system tumors; data analyses for these sites are under way. Within the next 12 months, two of the site-specific studies (salivary gland tumors and skin cancer) will be completed.

The two papers on salivary gland tumors were submitted for journal publication. Two draft papers on skin cancer will be submitted for internal review and journal publication. In addition, draft papers on central nervous system tumors and liver cancer will be prepared and possibly be submitted for internal review. Most of the site-specific studies now in progress should be completed within the next 3 or 4 years. New studies will be started on cancers of the colon/rectum and stomach. Other sites of interest will likely be identified.

RP 6-88 Comparative analysis of the LSS population and a cohort of 265,000 Japanese men and women. K Mabuchi, S Akiba, T Hirayama.

A large prospective cohort study conducted by Hirayama consists of 265,000 people selected from among residents of six prefectures in Japan, with a follow-up covering 16 years. The purpose of this RP is to undertake comparative analyses of data from the LSS and Hirayama cohorts with respect to smoking and other life-style factors related to cancer and other diseases. Knowledge gained from the analyses will lead to better understanding of the role of nonradiation risk factors which may be operative as confounding factors in the LSS risk assessment. Several papers on cigarette smoking and cancer have been published as a result of analyses conducted under this RP. Because of the recent death of Dr Hirayama, future collaborative arrangements are uncertain at the present time. 
RP 8-85 Incidence study on malignant and benign genital tumors among females, Hiroshima and Nagasaki, 1950-80. S Tokuoka S, K Kawai, Y Shimizu, K Inai, $\mathrm{K}$ Ohe, $\mathrm{K}$ Mabuchi.

Several reports suggest genital cancer as a late effect of local irradiation, but evidence of effects of whole body irradiation on genital cancers is limited. This RP intends to determine the incidence of tumors of various female genital organs in the LSS. A study of ovarian tumors has been superseded by RP 2-92, as reported elsewhere. It is planned that a separate RP be prepared for uterine tumors.

RP 14-79 Interaction between radiation dose and host factors. An epidemiological case-control study of female breast cancer in atomic-bomb survivors. K Mabuchi, JB Cologne, Y Shibata, M Tokunaga, CE Land.

This ongoing case-control study nested in the LSS cohort is intended to evaluate the effects, if any, of host factors other than age on radiation carcinogenesis in women. Host factors of interest have included traditional risk factors such as reproductive histories, ovarian activity, and use of exogenous hormones. In a recently published case-control study of 196 breast cancer cases and 566 controls, the risk was found to be positively associated with age at first full-term pregnancy, while negative associations were observed with number of births and total cumulative period of breast feeding. Significant positive associations were also found with histories of treatment for dysmenorrhea, and uterine or ovarian surgery. Neither age at menarche nor age at menopause was significantly associated with breast cancer. Radiation exposure (from the atomic bombs) was associated in a multiplicative manner with.age at first full-term pregnancy, number of children, and cumulative total period of lactation. More recently, special attention has been given to family predisposition because of the rapidly increasing knowledge on BRCA-1, -2 and ATM, and possibly other genes which may play an important role in heritable forms of breast cancer. Preparations are currently underway for conducting a new study of early-onset versus late-onset breast cancer cases to investigate family pedigrees and inherited mutations in the genes mentioned above using tissues and blood samples. A research protocol for this study will be presented within the next 12 months, and the study will be carried out over a 3- to 4 year period.

RP 4-75 Research plan for RERF studies of the potential genetic effects of atomic radiation: Hiroshima and Nagasaki. Part 1. Mortality study of children born to atomic-bomb survivors. Y Yoshimoto, K Mabuchi, WJ Schull, JV Neel.

This is a long-term mortality follow-up of a cohort of 77,000 children born to parents, either one or both of whom were exposed to radiation from the atomic bombs, and to control parents. As with the LSS, the mortality follow-up is based on the 
ascertainment of deaths through the nationwide family registration; the Hiroshima and Nagasaki tumor registries also provide cancer incidence data for a subset of the cohort. Previously published 1945-1985 mortality data for diseases excluding external causes showed no significant increase associated with parental radiation exposure. An apparent increase in noncancer mortality at attained ages $<20$ years in the $>2.5 \mathrm{~Sv}$ dose category was considered due to worsened socioeconomic conditions in which many survivors lived in the aftermath of the bombings. Tumor registry cancer incidence (through 1982) showed no excess cancer risk associated with parental radiation during the first two decades of life. Through 1990, 4130 deaths have occurred including 203 cancer deaths in about 67,500 with DS86 dose estimates. Through the tumor registries, 448 cancer cases were identified, including 60 leukemia and 28 lymphoma cases. Preliminary analyses showed no significant relationship between parental exposure and the risk of leukemia or lymphoma. We plan to complete the analysis of the latest mortality and cancer data within the next 12 months. The possibility of mail or other surveys will be explored in order to determine the whereabouts of each subject and to obtain information on socioeconomic and life-style characteristics as well as health status of each subject. Mortality and morbidity follow-up will continue so that potential effects of parental exposure on adult-type cancers and diseases can be studied.

RP 1-75 Research plan for RERF study of life span of A-bomb survivors, Hiroshima and Nagasaki. Y Shimizu, K Mabuchi, DL Preston, Y Shibata, DA Pierce.

This is a long-term follow-up of a fixed cohort consisting of 82,000 atomic bomb survivors and 27,000 nonexposed individuals, known as the Life Span Study (LSS) cohort. The follow-up began in 1950 using the family registration system, Koseki, which assures virtually complete ascertainment of mortality. It also serves as the sampling frame for a clinical subcohort, the Adult Health Study sample. Periodic analyses of the LSS mortality data have resulted in a series of reports, with the latest published report (Report 11) covering both cancer and noncancer mortality through 1985. Recently, through the tumor registries in Hiroshima and Nagasaki, it has also become possible to study cancer incidence in a subset of the LSS. The first comprehensive report of the LSS cancer incidence data was published in 1994. As of 1994 , about $50 \%$ of the subjects in the LSS sample are alive, and over $85 \%$ of those exposed at ages less than 30 years are alive. Because of the uncertainty of the current risk estimates based on the incomplete follow-up of those exposed early in life, continuation of the follow-up, in terms of both mortality and cancer incidence, is essential. The latest published LSS mortality data also demonstrated excess risk of death from noncancer causes, largely from cardiovascular and digestive diseases, associated with radiation exposure. Further research is needed to clarify the nature and magnitude of the excess noncancer risk. LSS Report 12 on cancer mortality updated through 1990 has been accepted by Radiation Research. Analyses of LSS noncancer 
mortality data through 1990 are also underway. Report 12 on cancer mortality is expected to be published in July 1996. (Editor's note: published in 146:1-27, 1996.) We plan to submit Report 12 on noncancer mortality in the coming year. Within the next three years, preparation will begin for the next LSS report which will include updated (possibly through 1995) incidence and mortality data.

RP 18-61 Tumor registry study in Hiroshima and Nagasaki. K Mabuchi, $M$ Soda, Y Fujita.

This RP provides the framework for operating the Hiroshima and Nagasaki tumor registries. The objective of these registries is to systematically collect and manage tumor cases in the populations of the cities of Hiroshima and Nagasaki. These registries are undertaken under the auspices of the medical association of each city, with technical support provided by RERF. The registries are linked with the Master File for the major RERF cohort samples (LSS, In-utero and $F_{1}$ ) and thus serve as the source of RERF cancer incidence studies in each cohort. The Hiroshima and Nagasaki tumor registries are the first population-based registries in Japan. Case ascertainment and data collection are based on abstraction of medical records conducted by trained personnel at regularly scheduled hospital visitations. This active approach is reflected in a high quality of incidence data not typically seen for other tumor registries in Japan, most of which rely on passive case notifications by physicians. The Hiroshima and Nagasaki tumor registry data have regularly been included in recent volumes of "Cancer Incidence of Five Continents" compiled by the International Association of Cancer Registries (IACR) at the International Agency for Research on Cancer (IARC) in Lyon, France. The latest cancer incidence from the two cities will be included in the next volume, Volume VII, of this monograph which is expected to be published within the next 2 to 3 years. The latest childhood cancer incidence data in Hiroshima and Nagasaki will be included in an upcoming edition of "International Incidence of Childhood Cancer," also compiled by IARC.

RP 29-60 Detection of leukemia and related disorders. $K$ Mabuchi, A Kuramoto, M Tomonaga, S Kusumi, H Nonaka, N Kamada, H Dohy, M Soda, Y Fujita, DL Preston.

This case-finding program, known as the Leukemia Registry, was started in 1948, with collaboration from hematologists and physicians involved in the treatment and care of leukemia patients in Hiroshima and Nagasaki. It is designed to detect, document, evaluate and diagnose cases of leukemia and certain closely related hematological disorders occurring among the LSS and other RERF cohorts. In the early years, case finding was based on a wide variety of sources including reporting from the participating physicians, clinical examinations carried out at $\mathrm{ABCC}$, death certificates, and obituaries. With the recent improvement in the Hiroshima and Nagasaki tumor 
registries, the ascertainment of leukemia cases currently relies primarily on the tumor registry's activities. In the mid-1980s, over $60 \%$ of leukemia cases in the Leukemia Registry were reclassified using modern diagnostic criteria and nomenclature, including the French-American-British (FAB) classification for acute leukemias. The latest published report resulted from comprehensive analyses of LSS incidence data for 1950 to 1987 , involving analyses of dose response and modifying effects for major leukemia types (acute lymphocytic leukemia, acute myelogenous leukemia, chronic myelocytic leukemia, adult T-cell leukemia) and lymphoma and multiple myeloma. An important question presently remaining relates to the risk for multiple myeloma (see RP 3-94). A new site-specific RP, which is to be undertaken jointly by hematologists and pathologists, has been developed to investigate all lymphoid tumors, including multiple myeloma, so that more definitive data be obtained on the risk of these tumors. This new lymphoid study will be a major focus of this RP in the next few years (see detailed plan under RP 3-94).

Tissue registry (no RP number). K Mabuchi, M Soda, Y Fujita.

Tissue registries, started in 1973, are undertaken under the auspices of the prefecture medical association in Hiroshima and the city medical association in Nagasaki. Pathology slides are collected for each tumor, malignant and benign, together with pathology reports. Tissue registry data are processed at RERF together with the tumor registry data from each city and entered into a common data base. These registries provide a supplemental source for ascertaining tumor cases for the tumor registries. They were especially valuable in the early years when there was difficulty in seeking collaboration from certain hospitals in Hiroshima. A large number of pathology slides collected and stored at one location greatly facilitate the conduct of a large number of site-specific studies involving pathology reviews of cases diagnosed over many years.

\section{Department of Statistics}

RP 5-87 Radiation-related damage to the developing human brain. WJ Schull, M Otake, K Masuda, H Nishitani, K Hasuo, I Goto, T Kobayashi.

A pilot project involving brain imaging of five severely mentally retarded people who were exposed in utero suggested that radiation exposure between the 8 th and 15 th week of gestation can lead to specific neuronal migrational errors in the developing brain. This study was intended to extend the initial pilot study by recruiting additional mentally retarded individuals who were exposed in utero. Efforts were made to extend the study to include additional low-IQ, in-utero-exposed subjects who are not mentally retarded. There has been little progress on these primary objectives. This is due in part to a concern about the nature of the procedures involved and the lack of any clear 
benefit to those who would participate in the project. At this time, the primary work related to this RP concerns summarizing and reanalyzing related data on mental and physical development of children exposed in utero, with particular emphasis on assessing effect modification associated with gestational age at the time of exposure. A review paper summarizing the full range of RERF data related to this issue is being prepared and should be completed within the next year. For the longer term, there is a need to decide if the original goals of this project can be met. The only RERF researcher involved in this project is Dr Otake, who will be retiring in 1996. If this projects is to be continued, it is necessary to determine who from RERF will be responsible for this project. Success will depend on the active involvement and support of the Department of Clinical Studies.

RP 8-89 Senile changes of the brain in Hiroshima and Nagasaki A-bomb survivors. $M$ Kishikawa, $M$ Otake, T Kobuke, $M$ Iseki, $H$ Kondo, $M$ Tokunaga, $H$ Fujii, I Nishimori.

This investigation will consider the late effects of radiation exposure on aging of the central nervous system by attempting to determine if observable changes of the brain may have resulted from atomic-bomb exposure. A total of 190 subjects, 95 each from the study and control groups (158 in Hiroshima and 32 in Nagasaki), will be selected for the current study with as close matching as possible on sex and age (for both age at exposure and age at death). Using special stains, serial sections will be prepared for the three brain sites (the hippocampus and parahippocampal gyrus, the tip of the frontal cortex, and the tip of the occipital cortex). These sections will be examined for senile plaques, neurofibrillary tangles, granulovacuolar degeneration, and such vascular lesions as amyloid angiopathy by pathologists without previous knowledge of the exposure status, age, sex, or underlying disease of each subject. The results will be examined for relationships to radiation dose and aging effects.

RP 4-86 Evaluation of index of physiological measurements: A predictor of mortality or morbidity associated with aging. S Fujita, H Sasaki, K Kodama, F Kasagi.

The study is intended to investigate how well physiological measurements made on more than 8000 Adult Health Study participants from 1970 to 1972 can predict morbidity or mortality. An index of physiological age has been constructed, and people were classified into three relative age groups according to whether their physiological age index was less than, similar to, or greater than their chronological age. Initial analyses suggest an association between the relative age and subsequent mortality, especially cardiovascular disease mortality, after allowance for other risk factors. Further analyses of the association between relative age, radiation exposure, and mortality or morbidity are needed. In the next year, a paper on the current analyses 
should be completed and submitted for publication. Over the next three years, additional analyses will be carried out and new data on grip strength currently being collected in the AHS examination will be used to examine the stability of the relative age index.

RP 7-76 The value of Adult Health Study family history records in the determination of genetic influences on the development of cancer and other disorders. JB Cologne, M Yamada, K Mabuchi.

This project was originally undertaken to ascertain the feasibility of using AHS family-history information for studying genetic influences on cancer and other diseases. For many years there was little or no activity on this project, and the data that were collected in the earlier years were not stored in computer databases. Recent advances in the understanding of genetic effects on disease, particularly cancer, together with the development of improved methods for genetic epidemiology and statistical genetics, have led to specific hypotheses concerning genetic epidemiology and radiation carcinogenesis which RERF is uniquely set to address through the combination of information in its tumor and tissue registries with its molecular genetics capabilities. Over the past two years, efforts have been made to revive and modernize this project. The short-term goal is to develop a family-relationship database for the RERF cohorts based on information from many sources. Once established, this database should serve as the basis for specific research projects, such as case-control studies or family history studies, with possible sampling of blood for molecular-genetic analysis (the design of a breast cancer study is being developed). With the aging of the RERF study population, it is becoming increasingly difficult to obtain the information needed for genetic studies, thus rapid progress in database development and study design is essential. Unfortunately, progress has been hampered by the loss, without replacement, of an epidemiologist and a database specialist interested in and knowledgeable of genetic studies and data management issues. Given current trends in radiation research, the development of the family-relationship database and the design and conduct of studies based on these data should receive high priority. While outside collaboration and support will be essential for the success of this effort, it is also critical that RERF be able to employ personnel with the appropriate epidemiologic and database skills to design and conduct family studies

RP 36-63 Blood groups in Adult Health Study and in utero ATB subjects, Hiroshima and Nagasaki. S Izumi, JL Ohara, DL Preston.

Current work on this protocol involves assessing phenotype and genotype frequencies in about 16,000 members of the LSS who are included in the AHS, about 1000 in utero subjects, and almost $26,000 F_{1}$ subjects examined at ABCC or RERF. At present, genotype frequencies are being estimated for the major blood groups. The 
analyses of these data include assessing departures from Hardy-Weinberg equilibrium and comparing the genotype distributions in the two cities and for the two generations of people examined by RERF. One or more papers on this initial work should be completed within the next year. Following the initial descriptive analyses, it is planned to link the blood group data to data on diseases diagnosed among AHS participants, cancer incidence data obtained from the tumor registries, and cause of death data obtained from routine mortality follow-up. A specific area of interest for future investigation concerns the relationship between blood groups and digestive diseases or cancer of the digestive organs.

RP 2-61 Study of mortality in children exposed in utero. $\mathrm{R}$ Delongchamp, $\mathrm{Y}$ Yoshimoto, K Mabuchi.

This research is aimed at characterizing the relationship between mortality or cancer incidence and radiation dose in people who were in-utero at the time of the bombs. Earlier analyses have focused on the 2700 members of the in-utero mortality cohort. During the past year, work has been completed to clarify the nature of the inutero populations studied by RERF. It was decided that the current analyses would be based on data from the combined in-utero mortality and clinical cohorts. This increases the number of in-utero subjects considered in analyses of mortality and morbidity by about $30 \%$. Analyses of mortality through the end of 1992 in the expanded cohort are nearing completion, and a paper on these results will be submitted for publication within the next four months. Analyses of data on cancer incidence in the combined cohort are in progress. It is anticipated that this work will be published within the next year. As the members of this cohort are approaching 50 years of age, death rates and cancer incidence are increasing rapidly. Thus, the ability to assess excess risks should improve, and it will be important to reconsider risks in this population in three to five years. A paper on cancer mortality has been completed and is currently being reviewed for publication. A second paper on general mortality and gestational age will be completed this summer.

RP 18-59 Shielding survey and dosimetry study. S Fujita, S Funamoto, K Katagami, GD Kerr, M Kohda, JL Ohara, DL Preston, T Watanabe.

The DS86 dosimetry system provides the dose estimates that are used as the basis of all current RERF analyses of radiation effects. The computation of individual DS86 estimates requires computer programs and data bases developed for the US-Japan Joint Committee for Reassessment of Atomic Bomb Dosimetry, additional computer programs developed at RERF, and extensive data on location and shielding at the time of the bomb for individual survivors. The system was originally installed on the ACOS mainframe computer that was removed from RERF about one year ago. Over the past year, the DS86 computer programs and data bases have been moved to the new 
workstation-based RERF computer system. Fundamental work on installing and validating the DS86 system on the new computers has been completed. Efforts are currently underway to organize the individual shielding history data so that it can be incorporated into the new RERF database system. A complete roster of all persons for whom DS86 estimates are available is also being developed and should be incorporated into the RERF database system within six months. In addition to the well-defined LSS cohort, this roster will include mothers of members of the in utero cohorts and parents of people included in the various $F_{1}$ studies carried out at $A B C C$ and RERF. Documentation of the implementation and use of the DS86 system at RERF is incomplete. Within the next year, a final version of this documentation should be completed. A small joint project involving the comparison of DS86 dose estimates with DS86-like dose estimates computed by Hiroshima University will continue for the next year or so. Over the past few years, it has become clear that there are some problems with both the DS86 neutron and gamma dose estimates. It is probable that a revised dosimetry system will be developed within the next three years. Implementing a new dosimetry system is likely to require a considerable effort on the part of the RERF staff.

\section{Department of Clinical Studies, Hiroshima}

RP 2-75 Research plan for the RERF Adult Health Study, Hiroshima and Nagasaki. K Kodama, S Fujiwara, K Neriishi, M Yamada, M Nobuyoshi, H Ueda, M Akahoshi, T Nishikawa, M Soda.

The primary purpose of this study is to determine the types of diseases and abnormalities in physiologically or biochemically determined values occurring as a consequence of A-bomb exposure, and to collate this information with other life experiences and death. Providing information on nonfatal events as well as fatal ones has long been one of the purposes of the Adult Health Study. The major research elements of the Adult Health Study can be categorized as (1) characterization of cancer types in relation to various confounders of radiation effects, (2) radiation-related noncancer diseases (thyroid disease, cataract, etc.), (3) benign tumors, (4) cardiovascular disease, (5) aging, (6) medical dosimetry, (7) psychosocial changes associated with exposure, and (8) radiation- or age-related changes in other physiological endpoints.

Since the information on disease morbidity has mainly depended on the findings obtained at the time of examination, the maintenance of the participation rate has been an essential factor in the quality control of the AHS program. However, because of natural aging processes, it is anticipated that the participation rate will fall significantly in the near future, resulting in less complete data collection on morbidity. To improve health information retrieval, a mail survey followed by telephone contacting of nonrespondents was introduced in August 1995 in Hiroshima. The response rate to the 
new surveillance system in the first six months was $86.3 \%$, which was extremely good. This was also true for AHS members who refused to participate in the biennial medical examination and those who migrated out of the contact area. Their response rates were $70.1 \%$ and $81.4 \%$, respectively. Moreover, the response rate of those who were over 70 years old, in which age group the participation rate of medical examination has been poor, was $86.8 \%$. In addition, a substantial amount of information on newly developed diseases was obtained from this surveillance program. The above results suggest that the data collection on morbidity has become more complete upon the introduction of this new data collection system and that future analysis of data on morbidity of various diseases may give answers regarding the relation between morbidity and previous exposure to ionizing radiation.

Special emphasis has been placed on screening for skin, breast, and thyroid cancers which are often not fatal. Although the Adult Health Study sample is limited in size, exposures to other carcinogens can be obtained through personal interviews and through measurements using serum. A case-control study will be conducted on various cancers related to nutrients and potential carcinogen and/or promoter substances using the serum stored for analysis in the future.

Various benign tumors, such as uterine myoma, ovarian tumor, prostatic tumor and liver hemangioma, are being detected using abdominal ultrasonographic techniques in Hiroshima.

Storage of serum and other biological materials has been a high priority of the AHS. Routine collection and storage of lymphocytes from AHS participants was initiated in June 1990 in collaboration with the Department of Radiation Biology.

A study to assess the frequency and nature of the social and psychological problems experienced by the survivors is in progress using accumulated data. It is not known whether psychological problems themselves ultimately influence the magnitude of the risk through the adaptation to life-style changes.

In order to clarify pathogenic mechanisms of radiation-induced diseases, molecular epidemiological studies on the AHS population will be initiated with interdepartmental collaboration. For this study, collection of fresh biological materials from surgery, such as tissues of cancers, benign tumors, blood vessels and skin, is desirable through a more intensive morbidity surveillance, in addition to the current preservation of lymphocytes of the AHS subjects.

There have been many special clinical studies conducted in the Adult Health Study population. A summary of the ongoing studies will follow. 
1) RP 2-95 Pilot study: Characterization of monoclonal gammopathy by studying the role of the BSAP gene in CD19 antigen expression. M Nobuyoshi, K Neriishi, S Kusumi, T Seyama, M Kawano, E Nakashima, K Kodama, M Akiyama, A Kuramoto.

The objective of this pilot study is to develop sensitive cellular and molecular methods for characterizing benign monoclonal gammopathy by studying the role of B-cell lineage-specific activator protein (BSAP) for CD19 antigen expression in order to identify in the near future those A-bomb survivors who are most probably in transition to multiple myeloma.

2) RP 9-92 Study of liver diseases in the Adult Health Study sample: Relationship between radiation dose and infection by $B$ and $C$ hepatitis virus. S Fujiwara, S Kusumi, K Kodama, K Neriishi, H Nonaka, M Akahoshi, M Akiyama, S Akiba, K Mabuchi, JB Cologne, Y Hasegawa.

The most recent incidence study on noncancer diseases in the AHS has demonstrated a significant dose response in the incidence of chronic hepatitis and cirrhosis. To determine whether infection of hepatitis $\mathrm{C}$ virus (HCV) or hepatitis $\mathrm{B}$ virus (HBV) contributes to an increased incidence of chronic liver diseases in relation to radiation dose, $\mathrm{HCV}, \mathrm{HBs}$ antigen and antibody, and $\mathrm{HBe}$ antigen are being measured among all AHS participants in Hiroshima and Nagasaki.

3) RP 6-92 Establishment and operation of a system for collecting and storing leukemia cells. M Nobuyoshi, S Kusumi, K Kodama, K Mabuchi, M Akiyama, H Dohi, N Kamada, A Kuramoto.

Through this system, leukemia cells derived from bone marrow and peripheral blood from leukemia cases occurring among Life Span Study participants are being collected and stored to perform molecular biological studies of the developmental mechanism of leukemia due to atomic-bomb radiation.

4) RP 5-92 Study on senile dementia among the Adult Health Study subjects in Hiroshima and Nagasaki. K Kodama, M Yamada, M Akahoshi, S Nakamura, S Nagataki, F Kasagi, Y Shibata, LR White, K Oishi, H Sasaki, M Tsujihata, M Seto, Y Shimizu, JL Ohara, WJ Schull.

The objective of the senile dementia study is to determine if there is an association between exposure to the ionizing radiation and the subsequent impairment of cognitive function and the occurrence of senile dementia. This study will also provide crosscultural comparisons of the prevalence of senile dementia, since the same neuropsychological test batteries and the same criteria for the diagnosis are used in ongoing cohort studies in Honolulu and Seattle. 
5) RP 5-91 Radiation-therapy-cancer among Life Span Study subjects (addendum to RP 7-81). K Kato, S Antoku, S Sawada, K Kodama, S Kawamura, K Mabuchi.

RP 7-86 Doses to Adult Health Study participants from RERF radiological examinations, Hiroshima and Nagasaki. M Yamada, S Fujita, K Kato, K Kodama.

RP 8-86 Ionizing radiation for medical reasons reported by Adult Health Study participants, Hiroshima and Nagasaki. M Yamada, S Sawada, S Fujita, K Kodama.

RP 8-87 Organ doses from medical X-ray exposures (addendum to RP 884). K Kato, S Sawada, S Antoku, WJ Russell, S Fujita, K Kodama, M Yamada.

In assessing A-bomb radiation effects, the contribution of medical radiation exposures must be considered. The information concerning the therapeutic and diagnostic radiation exposures has been obtained by interviewing the AHS participants. To determine the organ doses, some medical radiation exposures have been experimentally reproduced using technical exposure factors obtained by hospital and clinic surveys. Information on medical radiation exposure is useful for determining the relative contribution of medical and A-bomb radiation doses to the A-bomb survivors.

6) RP 3-91 A comparative study of vertebral fracture prevalence among Japanese, Japanese-Americans in Hawaii, and Caucasians in Minnesota. $S$ Fujiwara, PD Ross, LJ Melton II, C Huang, JW Davis, RS Epstein, RD Wasnich, K Kodama.

RP 3-89 Osteoporosis in Hiroshima atomic bomb survivors. S Fujiwara, DJ Pawel, K Kodama, M Fukunaga, $\mathrm{H}$ Orimo.

The objective of osteoporosis study is to determine if previous exposure to radiation influences the occurrence of aging-related disorders. Osteoporosis is a common consequence of aging, and bone loss is influenced by menopause and parathyroid hormone levels which are radiation related. Bone mineral density is being measured among the AHS participants using Dual X-ray absorptiometry. Measurements of an individual's bone mass and temporal changes provide the relationship between radiation and aging. 
7) RP 3-90 The association of serum cholesterol with noncardiovascular mortality and morbidity in the Adult Health Study population. M Yamada, F Kasagi, K Kodama, S Fujiwara, M Soda, H Sasaki.

The previous analysis showed that the serum cholesterol level in exposed people was significantly elevated compared to that in the nonexposed. The major objectives of this study are to assess the association of serum cholesterol with subsequent mortality and morbidity and to evaluate the effects of ionizing radiation on such an association.

8) RP 2-89 Hypercalcemia in A-bomb survivors, Hiroshima and Nagasaki (addendum to RP 11-86). S Fujiwara, E Nakashima, RR Delongchamp, H Ezaki, K Kodama, M Tsuruta, M Shiraki.

RP 11-86 Prevalence of hyperparathyroidism in atomic-bomb survivors during AHS cycle 15, Hiroshima and Nagasaki. S Fujiwara, E Nakashima, RR Delongchamp, H Ezaki, K Kodama, M Tsuruta.

A radiation-related increase in occurrence of hyperparathyroidism is one of the most recent findings found to be clearly associated with ionizing radiation determined in a prevalence study. Continuation of this study should provide not only incidence data of hyperparathyroidism but also a clue for determining the cause of slightly elevated levels of serum calcium, parathyroid hormone, and calcitonin among those exposed to higher doses. In addition, a molecular biological study of parathyroid adenoma has been initiated with cooperation of the Department of Radiobiology to clarify the pathogenic mechanism.

9) RP 1-89 The prevalence of radiation-related skin lesions in the Adult Health Study population, Hiroshima-Nagasaki. M Yamada, S Yamamoto, H Yoshida, K Kodama.

Studies of skin cancer among persons exposed to radiation due to occupational or medical therapeutic exposures have shown that skin is vulnerable to radiation carcinogenesis. In this study, dermatological examinations are performed during the biennial AHS health examinations to detect not only skin cancer but also precancerous lesions such as senile keratosis. Information concerning UV-rays of burn and trauma scars will be considered in the analysis.

10) RP 6-86 Ultrasonographic screening of Adult Health Study participants to detect cancer and other diseases. $K$ Kodama, $S$ Kawamura, $S$ Fujiwara, $M$ Akahoshi.

The increased incidence of uterine myoma among heavily exposed subjects was reported in AHS report 7. In order to avoid potential bias of diagnosis, abdominal 
ultrasonographic examination was conducted in Hiroshima between 1992 and 1993. The prevalence of uterine nodule increased significantly with radiation dose, with an odds ratio of 1:6 for those exposed to $1 \mathrm{~Gy}$. This result suggested that benign tumors such as uterine myoma may be induced by radiation exposure, although the pathogenesis remains unclear. Further studies are needed to clarify whether the association is induced by radiation or not.

11) RP 5-86 Dietary habit survey using a simple and computerized diet survey system (addendum to RP 8-83). M Yamada, H Hayabuchi, K Kodama, H Sasaki, Y Fujita.

Dietary factors are considered to be confounding factors of radiation effects in the A-bomb survivors. However, in the past, only old fashioned dietary habit surveys, including 24-hour recall surveys, had been conducted. Recently, simple and accurate methods using a computerized diet survey system have become available and the nutritional factors obtained by this system can be used to analyse risk factors on prevalence or incidence of certain diseases in the A-bomb survivors.

12) RP 6-85 Study of M-proteinemia in the Adult Health Study sample (addendum to RP 9-79). $K$ Neriishi, $Y$ Yoshimoto, $R$ Carter, $S$ Kawamura, $K$ Iwato, M Kawano, K Fujimura, A Kuramoto.

Uncertainties regarding the apparent dose-related increase of multiple myeloma exist. Clinical studies to detect a precursor state of multiple myeloma by protein electrophoresis have been employed in the AHS biennial examinations since 1985. A previous study revealed a slight increase in the prevalence of benign monoclonal gammopathy. At present, an incidence study is being conducted for aging subjects who are at highest risk of monoclonal gammopathy.

13) RP 4-85 Incidence and risk factors of coronary heart disease (CHD) in Japanese men living in Japan and Hawaii. $K$ Kodama, $Y$ Shimizu, F Kasagi, $H$ Ueda, M Yamada, H Sasaki, D Curb, B Rodoriguez, K Yano.

There are two major objectives in this research protocol. The first objective is to study the morbidity, mortality and the risk factors of cardiovascular diseases (CVD) in the NI-HON-SAN (Nihon-Honolulu-San Francisco) cohort of the AHS and compare the findings with those in the cohort of the Honolulu Heart Program in Hawaii.

The second objective, which is more important for RERF, is to study the relation between radiation exposure and CVD in the entire AHS cohort. The epidemiological methodology developed by this project has been effectively utilized to investigate radiation effects on CVD in the A-bomb survivors. The most recent finding on radiation and CVD is that a weak but very consistent association between radiation 
dose and various endpoints of atherosclerosis was observed. These endpoints include myocardial infarction, thromboembolic stroke, calcification of aortic arch, retinal arteriosclerosis, isolated systolic hypertension, and abnormality of pulse wave velocity.

A study on ankle-arm blood pressure ratio is currently underway in Hiroshima. A new study on titers of Chlamydia pneumoniae and serum levels of homocysteine as a confounder of radiation effect on CVD will be initiated in the near future.

\section{Department of Clinical Studies, Nagasaki}

RP 6-89 Incidence of radiation-related skin lesions in the Adult Health Study populations of Hiroshima and Nagasaki, 1958-89. $T$ Nishikawa, $M$ Yamada, $K$ Kodama, Y Hosoda, K Shimaoka, K Mabuchi, M Soda, N Sadamori, M Kishikawa, DE Thompson, SC Finch.

Specimens are still being collected at present, and the study is expected to require at least one more year for completion.

RP 5-92 Study of senile dementia among the Adult Health Study subjects. K Kodama, K Oishi, M Yamada, H Sasaki, M Akahoshi, K Shimaoka, F Kasagi, Y Shimizu, Y Shibata, JL Ohara, S Nakamura, M Tsujihata, S Nagataki, M Seto, LR White, WJ Schull.

This study is being conducted for four years (from 1 December 1993 to 30 November 1997). A total of 859 cases have been examined to date, leaving approximately 500 cases to be investigated.

RP 4-93 Epidemiologic study of anti-adult T-cell leukemia-associated antigen antibodies in the Adult Health Study sample, Nagasaki. K Oishi, Y Shibata, M Akahoshi, T Nakamura, S Nagataki, M Tsujihata, T Matsuo, M Tomonaga, $\mathrm{K}$ Shimaoka.

This study has already reached completion and has received approval for publication. At present, the manuscript is being prepared for submission to a journal.

In this study, the association of human T-lymphotropic virus type I (HTLV-I) with autoimmune disorders was investigated on the basis of prevalence of antinuclear antibody (ANA), rheumatoid factor (RF) and anti-thyroglobulin antibody (anti-Tg) and immunoglobulin (Ig) serum level (IgG, IgA and IgM). The mean serum level of IgM was higher in HTLV-I-seropositive subjects than in HTLV-I-seronegative subjects, and a significant association with HTLV-I $(P=0.01)$ and sex $(P=0.02)$ was observed in the IgM serum level after adjustment for age and radiation dose. No association with HTLV-I was noted in the prevalence of auto-antibodies except ANA, which showed a lower prevalence in HTLV-I-seropositive subjects than in HTLV-I-seronegative subjects. These results suggest the existence of some clear humoral immunity 
difference between HTLV-I infected and noninfected subjects, but whether HTLV-I infection can lead to autoiummune disorders remains uncertain.

RP 1-95 Effects of menopause on risk factors for ischemic heart disease: A longitudinal study of the Nagasaki Adult Health Study sample. M Akahoshi, M Soda, M Tsuruta, TP Rose, E Nakashima, M Saimei, S Seto, K Yano.

This study was initiated in January 1995 on about 80 subjects. At present the second collection of blood samples is underway. The study will be continued until one year after the subjects experience menopause.

\section{Department of Genetics \\ Biochemical Genetics Laboratory}

RP 5-85 Culture of permanent lymphocyte cell lines as sources of biological samples for investigation of genetic effects of radiation on children of atomicbomb survivors. $C$ Satoh, $N$ Takahashi, $K$ Hiyama, M Otake, $M$ Akahoshi.

Our goal is to establish cell lines from B-lymphocytes from 1000 families composed of father-mother-child trio using Epstein-Barr virus transformation. In half of the families, one or both parents were exposed to A-bomb radiation of more than $0.01 \mathrm{~Sv}$ gonadal dose calculated with the relative biological effectiveness (RBE) of 20 ; the remaining half will be the controls. Intact lymphocytes and granulocytes are also stored in liquid nitrogen for confirmation of mutations. In 1985, we selected 1525 families using the T65 dose revised (T65DR) system. We have established cell lines from 963 families. Among the 963 families, 835 families are usable for our future study because DS86 is available for both parents or DS86 is available for one parent and the other parent was either NIC or the other parent was $3000 \mathrm{~m}$ or more from the hypocenter ATB. New families for which parental DS86 doses are available will be selected and cell lines will be established keeping the original goal of 1000 families in mind. However, success of this additional work depends on the availability of help mainly from the Nagasaki Department of Clinical Studies, who are short staffed.

RP 7-85 Study to develop methods of DNA analysis for detecting mutations in children of atomic-bomb survivors. $C$ Satoh, $N$ Takahashi, M Kodaira, J Asakawa.

To examine efficiencies of techniques and to compare various types of DNA as potential targets for detecting mutations, we have been conducting pilot studies in which we are examining 50 exposed families with 64 children and 50 control families with 60 children as a subsample study. Techniques for screening for nucleotide substitution mutations were too inefficient compared to the required number of nucleotides to detect a significant difference between the two groups. To detect the insertion/deletion/rearrangement $(\mathrm{I} / \mathrm{D} / \mathrm{R})$ type mutations believed to predominate 
among radiation mutations, we are examining repetitive sequences and single-copy sequences. No significant differences were detected in the mutation rates at the repetitive sequences including six minisatellites and six microsatellites between the two groups of children. DNA fingerprints composed of multiple minisatellites will be examined in the next year by multilocus probes in order to increase the power of our study. For mutations in single-copy sequences, we have developed two techniques. One is a new two-dimensional gel electrophoresis (2-DE) technique; the other is quantitative analysis searching for a $50 \%$ decrease or increase in intensity of chemiluminescent bands on a Southern filter.

We have started a pilot study to assess detectability of germ cell mutations in mice using the 2-DE method. From a mouse (BALB/c) DNA sample digested with a set of three restriction enzymes, approximately 1000 spots are detected on a single gel. Of these, 700 spots are usable for detecting the I/D/R type mutations. Each of the DNA samples from 36 offspring of the control mice and from 41 offspring of the male mice irradiated by 5-Gy $\mathrm{X}$ rays, the offspring being derived from irradiated spermatogonia, was digested with two different sets of restriction enzymes separately, and the resulting two digests were independently electrophoresed 2-dimensionally. A total of 154 ( $=77$ $\times 2$ ) gels are being analyzed for the $I D / R$ type mutations. Offspring of male mice irradiated by $3-G y \mathrm{X}$ rays are being collected and their DNA samples will also be examined by $2-\mathrm{DE}$. Results will be obtained in one year. For the quantitative analysis of Southern bands, the best conditions to detect $50 \%$ change in the band intensity have been decided for 21 probes that can detect loci which are human counterparts of the mouse seven specific loci or other loci located nearby. Using the two techniques, pilot studies will be conducted on the 100 families and genetic variants or mutants if any will be characterized. For quantitative analyses of data which will be obtained from these studies, we require assistance from the Department of Information Technology.

\section{Cytogenetics Laboratory}

RP 8-93 Cytogenetic study in the Adult Health Study population by fluorescence in situ hybridization. Y Kodama, M Nakano, $\mathrm{K}$ Ohtaki, AA Awa, DJ Pawel, N Nakamura.

This study using fluorescence in situ hybridization (FISH) is expected to provide answers to several questions raised by the past cytogenetic studies using the conventional staining method. These questions include city differences among those who were exposed in Japanese houses, different dose responses by different shielding categories primarily in Nagasaki, and possible bias in cytogenetic dose estimation due to clonal expansion of aberration-bearing cells. Because previous studies were not free from laboratory bias, a whole FISH study must be achieved in Hiroshima laboratory. Initially, it was planned to test 200 survivors (120 Hiroshima +80 Nagasaki survivors) a year. We hope to initiate sampling Nagasaki cases sometime in FY1996. Within 3 
years, nearly 500 Hiroshima survivors and 150 to 200 Nagasaki survivors will be examined. High-dose parents of progeny in the DNA study will also be introduced into this study. Further, nearly 150 survivors who donated their teeth extracted for medical reasons will be FISH examined to compare with tooth enamel ESR data.

RP 10-86 Radiation dose estimates using tooth samples. Part 1. Collection of tooth samples from A-bomb exposed people in Hiroshima and Nagasaki. $\mathrm{N}$ Nakamura.

The purpose of this research protocol is to collect A-bomb survivors' teeth extracted for medical reasons. RERF has not been involved in Nagasaki collection because Nagasaki University group had already sent letters to proximally exposed individuals before this protocol was approved in 1986. On the average, 20 to 30 teeth have been continuously received every year from Hiroshima survivors, giving rise to 500 specimens. About $30 \%$ to $50 \%$ of the collected materials are suitable for subsequent study.

RP 1-92 Radiation dose estimates using tooth samples. Part 2. Use of electron spin resonance on tooth enamel from Hiroshima atomic bomb survivors. $\mathrm{N}$ Nakamura, C Miyazawa, AA Awa.

Electron spin resonance (ESR) measurements will be conducted for 100 tooth samples collected from Hiroshima survivors. To estimate effects of dental X-ray exposures, each tooth is divided into two (lingual and facial) halves, followed by independent enamel isolation and ESR measurements. Because most of the conventional dental X-ray exposure comes from outside of the mouth, facial halves may possibly produce a larger ESR signal than lingual ones. Indeed, this was the case for about 10 samples out of 80 . Current ESR data for inner halves showed a good correlation with chromosome aberration data (ie, stable-type aberration frequency) with a correlation coefficient of $r=0.87$, in contrast to $r=0.73$ for chromosome aberration for the DS86 dose. The ESR measurements will be completed within 12 months. After that, additional gamma irradiation of known doses will be conducted to convert the initial ESR signal intensity to dose, which will take another year or two. Meanwhile, cytogenetic tests will be performed for as many tooth donors as possible so that the two independent measurements may be directly compared. It should be added that, when this research protocol was discussed, we did not have an ESR machine at RERF and thus the sample number was fixed to 100 for collaboration with outside scientists. In 1995, an ESR machine was installed at RERF and now an additional 100 samples or so are planned for the ESR measurements within a year or two.

Very recently, we completed the measurements of both halves for the initial 100 samples. We found that most of the discrepant cases (ie, those whose outer halves gave a considerably larger signal than the inner ones) were front teeth. The reason is not 
clear, but the results are in accord with the information provided by a group headed by Dr P Jacob in GSF, Germany.

\section{Department of Radiobiology}

RP 7-93 Molecular analysis of thyroid cancers among atomic-bomb survivors. T Seyama, T Mizuno, KS Iwamoto, M Akiyama, Y Shimizu, S Tokuoka, K Mabuchi.

Recent studies have shown that a fraction of thyroid cancer tissue contains a dominantly acting $R E T$ oncogene. We have found and published the fact that rearrangement to the activated form of the RET oncogene is possible in cell lines $\mathrm{X}$ irradiated in vitro. The present study tests a hypothesis that the increased risk of thyroid cancer among the atomic-bomb (A-bomb) survivors is at least partly due to induction of mutational changes, such as DIOS170-RET rearrangement. In addition, 19 tissue blocks of thyroid cancers of children from areas contaminated by the Chernobyl accident were obtained. Activated RET oncogene was detected in four of seven cases from which RNAs were successfully extracted; a high rate compared with published data. This suggests that some of the thyroid cancers in children from contaminated areas developed as a result of their radiation exposure. About one year is needed to complete collection of thyroid cancers for molecular analysis among A-bomb survivors. After preparing samples, we will start molecular analyses in the next two years because we have already established the techniques.

RP 3-93 Molecular analysis of skin cancers in atomic-bomb survivors. $T$ Mizuno, T Seyama, KS Iwamoto, M Akiyama, Y Fujita, Y Shimizu, M Tokunaga, M Kishikawa, S Tokuoka, K Mabuchi.

The purpose is to investigate the roles of the ras oncogenes and $p 53$ tumor suppressor gene of skin cancer in atomic-bomb survivors. In reports regarding solid tumor incidence in A-bomb survivors, an elevated risk of nonmelanoma skin cancer has been found among the survivors. Furthermore, analysis of histological type suggests that basal cell carcinoma is associated more commonly with radiation than squamous cell carcinoma. It is also well known that skin tumors generally arise in sun-exposed areas of the body. However, all areas on the skin among A-bomb survivors should have had an equal chance of being targets for molecular lesions of ionizing radiation. Our general objectives are to identify the mutational changes caused by the A-bomb radiation and the differences in the spectra of mutations induced by ionizing radiation and UV-light. All samples which are to be analyzed in this study have been collected. Several tumors had multiple mutations within exons 5 to 8 of the p53 gene. There appears to be a difference in mutation spectrum between control and highly exposed groups. Within the next 12 months, we project the completion of the mutation analyses of $p 53$ in these cases. Within the forthcoming two years, we hope to 
complete molecular analysis of the ras oncogenes and the entire $p 53$ tumor suppressor gene.

RP 2-93 Development of assay for somatic mutation at the locus of the neutrophil Fcy receptor III gene and preliminary study of atomic-bomb survivors. Y Kusunoki, Y Hirai, S Kyoizumi, T Suzuki, S Fujita, M Akiyama.

To establish a new somatic mutation assay system, with molecular analysis capabilities, we developed the neutrophil Fcy RIII locus assay and evaluated its suitability for biological dosimetry in A-bomb survivors. The frequency of mutant neutrophils (Mf) lacking expression of NA1 antigen was measured for about $500 \mathrm{~A}$ bomb survivors. Over the next 12 months, a multiparametric analysis will be made taking into account the effects of dose, age, smoking, medical radiation exposure, etc. A technical report will be prepared. Over the next 3 years, analysis of mutant cells at the gene level will be attempted using RT-PCR and PCR-SSCP techniques.

RP 1-93 Repertoire of T-cell antigen receptors and activity of hematopoietic progenitor cells in peripheral blood of atomic-bomb survivors (addendum to RPs 3-87, 4-87, 7-89). Y Kusunoki, T Suzuki, S Kyoizumi, Y Hirai, M Akiyama, S Fujita.

The repertoire of T-cell-receptor (TCR) variable-region (V) genes of mature T cells in peripheral blood of $1280 \mathrm{~A}$-bomb survivors is being studied by flow cytometry using antibodies against the products of TCR V genes and by T-cell responsiveness to superantigen staphylococcal enterotoxin. Peripheral-blood progenitor cells are also being measured using flow cytometry and the hematopoietic colony assay. These studies address the late effects of radiation on the generation of T-cell diversity and the production of hematopoietic cells. Approximately 1000 AHS subjects have been examined so far, and preliminary statistical analyses were performed for the stem cell data. The number of peripheral blood stem cells is significantly higher in males than in females, and their number decreases with age. Only in females was there a significant increase of the granulocyte stem cells with exposure dose. This finding is consistent with the AHS report that the number of leukocytes increases with dose in females, suggesting that myelopoiesis is promoted in women among the high-dose group. Some regulatory factors, such as cytokines stimulating myelopoiesis, may be promoted in women with high-dose exposure, although the reason for such a sex difference is unknown at present. Over the next 12 months, we will complete the measurement for all subjects and prepare several manuscripts for these studies. Based on the preliminary analysis of stem cells as mentioned above, we will propose a new research protocol to measure the levels of cytokines in the serum of the same subjects. 
RP 7-92 Molecular analysis of the $p 53$ tumor suppressor gene in breast cancers of atomic-bomb survivors (with Addendum).

Addendum to RP 7-92: Molecular analysis of $R b, P H B$, and other tumorsuppressor genes in breast cancers among atomic-bomb survivors. $T$ Seyama, $T$ Mizuno, KS Iwamoto, N Nakamura, M Akiyama, M Tokunaga, S Tokuoka, Y Fujita, K Mabuchi.

There is an increased risk of breast cancer among the A-bomb survivors, especially among those younger than nineteen at the time of the bomb. To date, however, there is no knowledge about the mutational events which are directly involved in radiation carcinogenesis. The $p 53$ gene and other genes which include but are not limited to $R b$, $P H B, B R C A 1$, will be examined regarding development of breast cancer. We established methods to investigate $p 53$ gene alterations, and as soon as the samples become available for these studies, we will start the molecular analyses. Currently, arrangement for collection of tissue materials is ongoing. The agreements for use of materials were concluded between the outside institutes and hospitals, and the RERF, because many of the breast cancer tissues are preserved at outside hospitals and institutes in Hiroshima and Nagasaki. After collection of the materials, three years are sufficient to complete the molecular analyses.

RP 5-90 Primary liver cancer incidence study among atomic-bomb survivors, 1958-87: molecular analysis of HBV and HCV. KS Iwamoto, T Mizuno, T Seyama, M Akiyama, S Fujita, M Tokunaga, S Tokuoka, Y Fujita, K Mabuchi.

RP 2-94 Molecular analysis of hepatocellular carcinoma among atomic-bomb survivors. KS Iwamoto, T Seyama, T Mizuno, M Akiyama, M Tokunaga, S Tokuoka, T Fukuhara, M Yamamoto, H Itakura, T Ikeda, T Fujita, K Mabuchi.

RPs 5-90 and 2-94 are parts of the liver cancer study series being conducted on the atomic-bomb survivors at the foundation. The particular role of the Department of Radiobiology is the molecular analysis of the liver tissue with relation to hepatitis viruses $B$ and $C$, and various tumor suppressor genes, especially $p 53$. Our objectives for the next year are to extract and isolate RNA from the some 800 paraffin-embedded tissues for analysis of $\mathrm{HCV}$ and, with respect to $p 53$, to complete sequencing of the four hot-spot exons. Our objectives for the next three years are to complete analysis for $\mathrm{HCV}$ infection and to sequence the remaining six exons of $p 53$. In addition, we will continue our efforts to collect more samples. Hopefully, some trends in $p 53$ mutations (the existence of and the spectra of) and HBV (with HVC by the next three years) with regard to exposure status, sex, city, ATB, hepatocellular carcinoma $(\mathrm{HCC})$ grade, etc. will become evident within the next year. 
RP 4-90 Establishment of a method for HLA-DQ and DP gene typing using the polymerase chain reaction. $T$ Hayashi, $Y$ Hirai, $Y$ Kusunoki, S Kyoizumi, N Nakamura, T Seyama, M Akiyama, K Kodama.

Objectives of this study are to establish at RERF a method of human leukocyte antigen (HLA) genotyping and to test for possible population bias among the A-bomb survivors on the assumption that some people's ability to survive might have differed from that of others during the period of reduced immune function after the A-bombing. HLA-DQA1 and DRB1 genotyping methods have been established. DNA samples of 1018 A-bomb survivors in Hiroshima were analyzed by these genotyping methods. The statistical analysis of the results is now under way, and a technical report of this analysis will be submitted within a year. For the next 3 years, other HLA-class II (HLA-DQB and DPB) genotyping methods will be introduced. All HLA-class II genotypes will be performed for the stored DNA samples obtained from the same subjects as mentioned above. The results of the HLA typing will be analyzed in conjunction with immune responses, among the A-bomb survivors, to various microorganisms.

RP 2-90 Cryopreservation of blood cells from Hiroshima and Nagasaki Adult Health Study participants. Y Hirai, Y Kusunoki, K Hamatani, S Kyoizumi, T Seyama, K Kodama, M Akiyama.

Effects of A-bomb radiation have been studied for various endpoints. The analytic techniques have continuously improved over time. We expect that studies of hithero unmeasurable effects will become possible in the future. Because A-bomb survivors are reaching advanced ages, we proposed to cryopreserve live blood cells from all AHS participants in order to have an available source of material for future studies. We will continue to cryopreserve blood cells from about 2000 AHS participants every year.

RP 11-89 A pilot study for detection of somatic mutations at the HLA-A locus in lymphocytes. Y Hirai, S Kyoizumi, Y Kusunoki, T Suzuki, M Akiyama.

We have studied human somatic mutations using five different gene loci as markers: GPA in erythrocytes, HPRT, TCR and HLA-A in lymphocytes, and FC RIII in neutrophils. However, we have not yet made simultaneous comparative studies of various cells using a single marker. In healthy adults, no difference in the frequencies of mutant HLA T lymphocytes, B lymphocytes, and neutrophils was detected. No general trends were noted over the time period sampled, and the reproducibility was good. Over the next 3 years, we will continue to determine the mutant frequencies of various blood cells from $120 \mathrm{~A}$-bomb survivors and statistically analyze the late effects of A-bomb radiation on various blood cells with differing cell kinetics. Analysis of mutant genes will be attempted using molecular biological techniques. 
RP 7-89 Screening of stem cell mutation in lymphoid lineage among A-bomb survivors and its characterization. T Suzuki, Y Hirai, Y Kusunoki, S Kyoizumi, M Akiyama.

The purpose of this study is to identify individuals in whom mutations have occurred in hematopoietic stem cells due to the atomic-bomb radiation and to clarify the in vivo kinetics of the mutant stem cells by estimating frequencies of their progenies in different cell lineages. Two survivors have so far been identified as such individuals. In one case, mutants bearing the same alteration of HPRT gene were observed in both $\mathrm{T}$ and $\mathrm{B}$ cells at a high frequency $\left(10^{-3}\right.$ to $\left.10^{-4}\right)$, and each mutant had a unique pattern of TCR or Ig gene rearrangement. Interestingly, such mutants were scarcely detected in CD45RA ${ }^{-}$memory $\mathrm{T}$ cell population, indicating that $\mathrm{T}$ cells derived from the mutant stem cell have not been exposed to antigens in vivo for 46 years after the time of the bomb, if the mutant stem cell is assumed to have started differentiating into $T$ cells at that time. A manuscript describing these results will be submitted within a year. In the other case, the same chromosome aberration was observed in $10 \%$ of in vitro colonies from peripheral $\mathrm{CD} 34^{+}$cells as well as $\mathrm{T}$ and $\mathrm{B}$ cell colonies. This is the first evidence for a chromosome aberration occurring in a multipotent hematopoietic stem cell of an A-bomb survivor and indicates that a single stem cell can generate as much as $10 \%$ of the peripheral lymphocytes. The article describing this case has recently been published. Over the next 12 months, we will investigate whether $\mathrm{T}$ cells with this chromosome aberration are also observed more frequently in CD45RA ${ }^{+}$naive $\mathrm{T}$ cell population than in the memory $\mathrm{T}$ cell population. Over the next three years, we will continue the attempt to identify additional persons showing mutations or chromosome aberrations in stem cells and estimate the number of stem cells participating in hematopoiesis and lymphopoiesis in humans. These studies will be done in collaboration with the Cytogenetics Laboratory in the Department of Genetics.

RP 7-88 Study of somatic mutations at the glycophorin A locus in erythrocytes of atomic bomb survivors.

RP 9-89 Detection of erythrocyte mutations at the glycophorin A locus in Nagasaki survivors and in Hiroshima area poison gas workers (addendum to RP 7-88). S Kyoizumi, Y Hirai, Y Kusunoki, T Suzuki, T Hayashi, M Akiyama, N Nakamura, K Kodama.

The glycophorin A (GPA) mutation assay does not directly measure the mutations in cancer-associated genes; it reflects the probability of mutations of these genes. Recently our findings on 1200 survivors in Hiroshima and Nagasaki show that the dose response of the deletion type GPA mutation frequency (Mf) is very similar to that of solid tumor incidence. This indirectly supports the involvement of radiation-induced 
mutations in human carcinogenesis. The molecular analyses of mutant reticulocytes by RT-PCR demonstrated that A-bomb radiation mainly induces nonproductive-type mutations in the GPA gene, most probably gene deletion. Over the next 12 months, we will continue and expand the GPA Mf measurements in A-bomb survivors and other radiation exposed populations. An in vivo irradiation experiment using SCID-hu mice implanted with human hematopoietic stem cells will be attempted to obtain the dose response of GPA mutations and to analyze molecular changes of the GPA gene and its mRNA. We will also attempt to isolate erythroid progenitor cells from peripheral blood of A-bomb survivors and to expand erythroid cells in culture for the analysis of mutant GPA genes. With respect to our 3-year objectives, we will continue measuring GPA Mf for AHS participants (about 3000 survivors in total). Long-term follow-up of cancer development in survivors whose Mf is regularly measured may demonstrate the relationship between radiation-induced somatic mutations and cancer risks.

RP 7-87 X-ray radiosensitivity of lymphocytes in vitro from A-bomb survivors. Part 3: Transformation of $B$ cells by Epstein-Barr virus and their cryopreservation (addendum to RP 3-86). Y Hirai, Y Kusunoki, S Kyoizumi, T Suzuki, M Akiyama.

We have begun cryopreservation of lymphocytes and granulocytes as per RP 2-90. For this study, it was initially proposed to cryopreserve EB virus transformed B cell lines from high-dose and control survivors for future studies in cell biology such as those dealing with radiosensitivity. However, the usefulness of these B cell lines in many other fields including immunological studies has become apparent. Therefore, the study cohort has been expanded. To date, cell lines from a total of about 750 AHS participants have been cryopreserved for future studies. We will continue EBVtransformation of B cells from the same subjects of RP 2-90.

RP 3-87 Cellular immune function and its relationship to in vitro T-lymphocyte radiosensitivity and MN blood group locus mutation frequency in A-bomb survivors: Precursor frequency analysis of mitogen- and antigen-responsive blood lymphocytes. S Kyoizumi, Y Kusunoki, Y Hirai, S Umeki, KS Iwamoto, M Akiyama.

The measurements for almost all subjects have been completed. Manuscripts describing the results of statistical analyses are being prepared. During the course of this study, we found mature $T$ cells with rare phenotypes such as $\mathrm{CD}^{-} 8^{-}$and $\mathrm{CD} 34^{-} \mathrm{T}$ cells. Three articles describing $\mathrm{CD}^{-} 8^{-} \mathrm{T}$ cells in A-bomb survivors have been published. $\mathrm{CD}^{-} 4^{+}$cells were found to be mutant T cells lacking T-cell receptor $\alpha$ or $\beta$ chains (TCR mutants). Extensive studies have been conducted on radiation-induced in vivo and in vitro TCR mutations. These studies have demonstrated that the TCR mutation assay using flow cytometry is a sensitive indicator of recent radiation 
exposure and that the TCR mutant frequency is highly correlated to the frequency of unstable type chromosome aberration, which has been generally used for biodosimetry. Recently, we have improved the TCR mutation assay in order to apply this biodosimeter to victims immediately after radiation exposure accidents. The TCR mutation assay has already been applied to a group of individuals in the Chelyabinsk contaminated area and demonstrated significant increase of TCR Mf with estimated cumulative exposure dose. Over the next 12 months, we will continue measuring TCR Mf for exposed persons in Russia, Taiwan and China to evaluate in vivo mutagenesis induced by low dose-chronic radiation exposure. We will further characterize the improved TCR mutation assay. Furthermore, analyses of TCR mutant clones from Abomb survivors and other exposed people will be continued to detect molecular fingerprints of radiation exposure. In the next 3 years, we will continue the cellular and molecular characterization of TCR mutant clones. In particular, the molecular mechanism of in vivo expansion of mutant T-cells with a memory phenotype in Abomb survivors will be studied. These studies will provide important insights to both immunological and radiobiological fields.

RP 2-86 Collection of surgically removed cancer tissues from A-bomb survivors: Special reference to thyroid and breast cancers. $S$ Ban, $T$ Seyama, $T$ Mizuno, KS Iwamoto, M Yano, M Akiyama.

This project collects and cryopreserves radiation-induced thyroid and breast cancer tissues in order to have a resource at RERF for future studies on oncogene and tumor suppression genes. These tissues will be useful for preparing DNA and possibly mRNA for studies of oncogenes and other molecular biological aspects of cancer. Current status of the collection of surgical samples of thyroid and breast cancers in Hiroshima is: 41 cases of thyroid cancer (A-bomb $(+)=12$, A-bomb $(-)=12$, exposure status unknown $=17$ ) and 74 cases of breast cancer (A-bomb $(+)=36$, A-bomb $(-)=$ 24 , early entrant $=7$, exposure status unknown $=7$ ). Collection of a small number of specimens will not satisfy the requirements of any study which requires matching of cases and controls. Consequently, it may not be worthwhile to continue this small effort. RERF may create a platform research plan for molecular epidemiology through close collaboration with City and Prefectural Medical Associations to remind pathologists that the survivors' specimens are a common heritage of Hiroshima and Nagasaki. We should emphasize that such collaboration will be essential to provide financial support as is done for the Tumor and Tissue Registry. 
RP 18-81 Pathophysiology and radiation response of human thyroid cells in culture and in grafts in athymic nu/nu mice. $S$ Kyoizumi, M Nobuyoshi, $T$ Mizuno, M Yano, T Suzuki, T Hayashi, T Seyama.

The radiobiological study of humans has been hampered by a lack of suitable in vivo experimental models. To overcome this limitation, we have attempted to create models using small animals which maintain human normal tissues with their active functions. This project originally sought to implant human thyroid tissues into athymic nude mice, but recently severe combined immunodeficient mice (SCID mice) were found to be better recipients because the success rate of human tissue implantation is much higher in SCID mice than in nude mice. Thus, SCID mouse-human chimeras (SCID-hu mice) can be applied to not only human thyroid tissue but also various human epithelial and hematolymphoid tissues. At the last Scientific Council Meeting, we demonstrated the usefulness of a SCID-hu skin model for the study of radiationinduced human epilation and SCID-hu cord blood model for human hematopoiesis. Based on the results, within a year we will propose a new platform research protocol which will expand the study to include other human epithelial tissues. Over the next 12 months, we will characterize and improve the SCID-hu cord blood and skin models to apply them to human radiobiology. The mechanism of radioresistance of follicular stem cells in the skin model will be studied. Furthermore, for the thyroid model we will study radiation-induced mutagenesis in cancer-associated genes such as RET oncogene activation which is believed to be one of the gene alterations specific for thyroid carcinogenesis. For the next 3 years, we will attempt to induce oncogenesis in human implants such as leukemia, thyroid cancer and skin cancer by radiation exposure. These approaches will clarify the molecular mechanisms of human radiation carcinogenesis, which have not been clear from studies on the survivors.

\section{Information Technology Department}

The Research Information Center was reorganized and renamed the Information Technology Department (ITD), with sections and units reflecting the major areas of support provided in the new computing environment: systems administration, data management, and application support. Newly transferred to the ITD was the library, another information resource at RERF.

With the new networked system of Unix workstations and personal computers (PCs) in place and the mainframe having been removed in January 1995, final details related to the transition were handled, and taking advantage of the capabilities of the new system became the primary focus of computing-related activities for FY1995.

Over 60 PCs were purchased to provide researchers and support staff with greater access to software and information resources important for research, and to replace older computers that no longer could support present-day software. Inventories were 
checked to ensure that RERF maintains the legal number of licenses for software in use.

A more powerful workstation server was installed to better accommodate computation-intensive statistical analyses. In the Department of Genetics, a second workstation was introduced to facilitate image analysis of $2 \mathrm{D}$ gels. A second database server has been ordered and will be set up to handle database queries by research staff. With the number of database users growing, the number and sophistication of queries being submitted have increased, and this has overloaded the single database server also used by Master File and Tumor Registry staff for daily maintenance and update of data essential for the mortality and cancer incidence follow-up studies. It therefore is necessary to handle queries and maintenance work on different servers.

The speed of the communications line linking RERF to the Internet was increased from $192 \mathrm{Kbps}$ to $1 \mathrm{Mbps}$ to handle growing use of the Internet by RERF staff. In addition, a full feed for the Usenet newsgroups is now received. Four dial-up lines were set up to enable staff holding valid RERF computer accounts to access the computing system from home or other locations outside RERF.

Disk storage space on the network has nearly doubled to approximately $80 \mathrm{~GB}$. This is approximately six times the storage space available on the previous mainframe system and has allowed more data to be stored on disk instead of tape for easier access.

In the area of database management, refinements to the Master File and Tumor Registry portions of the RERF database were made throughout the year. This, together with the easier-to-use database, more user-friendly PC software tools, and user training, has enabled Hiroshima and Nagasaki Tumor Registry staff to become more self-sufficient and carry out work that previously had to be conducted by ITD staff on a request basis. Cooperative efforts with RERF departments have continued in a number of areas related to data management and database design planning, as described below.

ITD has provided assistance to the joint efforts of the Departments of Statistics, Epidemiology, and Genetics in clarifying the $F_{1}$ and in utero cohorts. This project is nearing completion, upon which ITD will incorporate the cohort definitions into the database. Together with the Departments of Statistics and Epidemiology, ITD began preparing database documentation designed to provide the end-user suitable information about what is in the database as well as information important for ensuring that the data are used in proper context. Assistance also was provided to the Department of Statistics in their efforts to convert and maintain on the Unix workstation platform the in-house developed software, externally developed software, and data that comprise the Dosimetry System 1986 (DS86).

With much of the new system hardware and software now installed and the mainframe deadline behind us, one of the primary goals in FY1995 has been to organize and provide training to users to make best use of the new resources available. This is an area that we have not addressed adequately in the past. A small training facility of four to five PCs was set up in the ITD, and several types of classes and 
seminars were organized. These covered topics such as basic PC software use; introduction of RERF network and security basics; electronic mail; word processing; World Wide Web; database standard query language (SQL); and SAS Access, the interface between the RERF database and the SAS statistical analysis system. Whenever possible, experienced users outside ITD were asked to participate in the organization and preparation of material for these classes to ensure that course content would be relevant to RERF user needs. Thirteen different courses were created and held on a regular basis during the year. The response was remarkable; nearly 700 participants attended these courses. This is evidence of the interest and desire to learn how to put software and computing resources to best use. An enormous amount of time and effort must be expended in order to prepare quality classes and material, but the knowledge gained by users and the improvement of skill levels contribute significantly to more effective and efficient staff work. Expanding training and informational seminars into other important topic areas is needed, and we will continue to devote considerable effort here.

With business computing operations running smoothly under the new system, transfer of financial archive files from previously used dedicated business computers was completed, and the rental agreement for the two remaining dedicated units was terminated in mid-1995. Conversion of the system to transfer financial information directly between RERF and the Bank of Tokyo-Mitsubishi Ltd. also was completed.

Over the past several months, ITD staff have been providing technical guidance to an RERF-sanctioned committee actively working on organization, content, and preparation of a whole new set of World Wide Web pages designed to replace the old, outdated pages.

RERF must not lose sight of its need to reach out to the communities with which it shares things in common. As a public service, ITD tries to promote use of computer networks and share its knowledge of computer technology. Given staff shortages, this is done on a very limited basis, with groups whose financial resources or technical contacts are limited. One example is guidance provided to the Nuclear Safety Research Association in Tokyo on technology and methods for creating its own World Wide Web (WWW) server and Web pages. A second example is introduction of email to the Hiroshima International School (HIS). That project successfully led to HIS gaining approval from its Board to obtain a commercial email account for the school. Currently, we are providing HIS an area for test development of WWW pages. RERF incurs no cost for these activities.

Technical staff shortages in the ITD have become critical. Computer usage has grown dramatically at RERF, and along with it, the reliance on computer and electronic information resources to conduct much of RERF's work. During that time, we have lost approximately $40 \%$ of our experienced systems administration and database management staff; one additional staff member has been on medical leave since the beginning of 1996. Systems administration and database management personnel 
cannot keep pace with research and support needs, and this is hindering progress in many research projects. Existing computer staff cannot be expected to maintain the pace, work style, and burden that results from taking short-term, stop-gap measures to try to make ends meet under this persistent "temporary" personnel shortage. 


\section{Research Directions}

Editor's note: This document was prepared for the Blue Ribbon Review Committee meeting held in February 1996.

\section{Introduction}

We, the Board of Directors, wish to express our appreciation to the Blue Ribbon Review Committee for the opportunity to share our views about the future directions of RERF's research programs.

RERF's research program is the most comprehensive study of a large $(>100,000$ person), well-defined population that includes all ages and both sexes subjected to an instantaneous and wide range of radiation exposures. Several major research foci have evolved over time. These currently include epidemiological studies of cancer mortality and incidence and noncancer mortality among the atomic-bomb survivors; ongoing clinical follow-up with the collection of biological specimens and longitudinal clinical measurements and data on morbidity in a fixed subset of the survivor population; genetic and epidemiological studies of the children of the survivors; and application of modern molecular, cytological, physiological and other technologies to the unique biological samples provided by the survivors in order to identify radiation-induced changes and to investigate the underlying biological mechanisms of these changes. The results of RERF's epidemiological studies are the primary basis for radiation protection standards throughout the world while basic biological research at RERF already has clarified issues previously unresolvable by physical dosimetric and epidemiologic approaches. With recent and continuing impressive worldwide advances in molecular genetics, RERF should be able to play a unique and central role in determining radiation's effect at the gene locus level.

At RERF, almost daily interaction among scientists from these different research disciplines is the leavening agent that leads to mutual scientific progress and the fulfillment of RERF's mission: to clarify the medical effects of radiation on humans and to contribute to the health and welfare of the A-bomb survivors.

The committee should be aware of the fact that about a decade ago, then DOE Director of the Office of Energy Research Alvin Trivelpiece recommended to the Scientific Council that RERF insure that its programs take advantage of the exploding molecular technologies in determining the basic events culminating in radiationinduced health effects. This and DOE's expressed wish for an in-depth examination of RERF's future research needs led to three workshops, Radiation Susceptibility, Immunology, and Carcinogenesis, held in 1988 and 1989 in response to the Council recommendations. It was then decided that three more workshops be developed in the areas of Gerontological Research, Human Germline Mutagenesis, and Health Monitoring. They were held in 1990, 1991 and 1993 respectively. (The recommendations of these workshops were sent to Dr Clarke earlier.) Many of these 
recommendations have been implemented, and RERF has strengthened its material and personnel support for these branches of research as will become evident in our further discussions.

The RERF Scientific Council in its annual scientific review has made important and far-reaching recommendations during its 20-year existence and has profoundly affected the course of the RERF program.

It is not necessary to belabor here the impact of RERF's program on the international radiation research community. However, a few points should be emphasized. Cancer mortality risk estimates derived from atomic-bomb survivor data have supplanted all other cancer risk-estimate sources. The latter now are substantiation of, rather than alternatives to, the atomic-bomb survivor-based assumptions. Worldwide, radiation protection standards for workers and the public are based on the survivor risk estimates, as promulgated in the 1990 recommendations of the International Commission on Radiological Protection. Risks associated with population exposures due to accidental radiation releases and individual exposures (for example, in probability of causation lawsuits) also are estimated based on atomicbomb survivor data. RERF studies have shown that fetal radiation exposures affect physical and mental development and as the in-utero cohort ages the nature of the excess cancer risks in this group are being clarified. RERF is also the primary source of information on the occurrence and nature of genetic effects in humans following radiation exposure.

Finally, we, the Board of Directors, must express our dismay at the way the RERF program has been held hostage in recent years to the shortage of DOE's funds mainly caused by the changing yen-dollar exchange rate that has necessitated severe budget cuts, resulting in precipitous and unbalanced staff reductions. We are aware that financial support of radiation biomedical studies, particularly in the US, has been declining; however, RERF has had to cope with the vagaries of currency fluctuations in addition to weathering recent trends in reprioritizing research funding. It is essential that the two governmental financing agencies find the means to provide a stable research environment rather than lurching from one crisis to the next. The fiscal support to RERF by the Japanese Government is now legislated through the Atomic Bomb Survivors Relief Law which was newly enacted on 1 July 1995.

RERF is a unique research enterprise, studying an unparalleled population unlikely to exist again. At this juncture, we have an incomplete understanding of the late effects of radiation exposure during childhood and are just reaching a point in which we can expect to gain important insights into the existence and nature of genetic effects. The pursuit of this important knowledge and the fulfillment of the comprehensive program goals outlined herein depend on the continued support of the RERF programs. 


\section{Epidemiology program}

\section{Excess risk of death due to cancer}

The follow-up of the Life Span Study (LSS) cohort including 93,000 atomic-bomb survivors is the center of the RERF epidemiology program. The follow-up is based on the family registries which assure virtually complete ascertainment of death. LSS Report 12 on cancer mortality among the LSS cohort from 1950 through 1990 is currently undergoing peer review and will be published in 1996. This report emphasizes how the excess cancer risks associated with radiation exposure depend on modifying factors such as sex, age at exposure, and attained age, along with the uncertainties associated with the use of these risks, especially for those exposed as children. The evidence to date suggests that solid cancer excess risks are likely to persist and excess rates will increase throughout life. Excess relative risks for solid cancers among those exposed as adults have remained fairly constant throughout their lifetimes. For people exposed in childhood, there is some evidence that relative risks have declined slightly; however, excess rates (ie, absolute risks) for this group are increasing with age in a manner consistent with the increases seen for those exposed as adults. For those exposed as children, the total number of cancer deaths is currently small; however, this number is doubling every 5 years, and estimates of the number of excess cases are increasing at about the same rate.

Although lifetime follow-up is complete for those exposed when older than age 50, more than half of the LSS cohort and over $90 \%$ of those exposed as children are alive now. The table below gives both the actual and projected numbers of persons in the cohort. By the year 2000 the number of cancer deaths among those exposed when

LSS cohort* size, 1950-2020

\begin{tabular}{lrrrrrrrr}
\hline \multirow{2}{*}{$\begin{array}{c}\text { Age at } \\
\text { exposure }(y)\end{array}$} & 1950 & 1990 & 1995 & 2000 & 2005 & 2010 & 2015 & 2020 \\
\cline { 2 - 9 } $0-9$ & 17,824 & 16,768 & 16,450 & 15,990 & 15,290 & 14,280 & 12,710 & 10,390 \\
$10-19$ & 17,557 & 15,163 & 14,500 & 13,540 & 12,040 & 9800 & 6780 & 3620 \\
$>20$ & 51,191 & 16,971 & 12,800 & 8910 & 5430 & 2710 & 970 & 100 \\
Total & 86,572 & 48,902 & 43,750 & 38,440 & 32,760 & 26,790 & 20,460 & 14,110 \\
\hline $\begin{array}{l}\text { Average at- } \\
\text { tained age (y) }\end{array}$ & 33.5 & 61.4 & 64.7 & 67.9 & 71.3 & 74.7 & 78.0 & 81.3 \\
$\begin{array}{l}\text { Average age } \\
\text { ATB (y) }\end{array}$ & 28.5 & 16.4 & 14.7 & 12.9 & 11.3 & 9.7 & 8.0 & 6.3 \\
\hline
\end{tabular}

"Restricted to persons with known DS86 dose

Note: $\mathrm{ATB}=$ at the time of the bombing 
younger than age 20 will be 3 to 4 times that seen up to 1990 and will continue to increase rapidly throughout the first 10 to 15 years of the next century. Therefore continued follow-up of those exposed as children or as young adults is essential to the understanding of radiation effects on cancer risks in this cohort.

Continued follow-up together with advances in analytical methods are making it possible to address more complex issues than in the past. RERF researchers are developing alternatives to the time-constant (given sex and age at exposure) relative risk models that play a central role in current descriptions of radiation effects on solid cancer risks. These alternative models help to quantify uncertainties in current risk estimates, especially for those exposed as children, and may provide useful insights into the nature of radiation carcinogenesis. Current data suggest that leukemia risks for those exposed as adults have persisted throughout life, whereas excess risks for those exposed as children have decreased with time. In view of the complex pattern of excess leukemia, continued assessment is necessary for a complete understanding of the leukemia risks following radiation exposure. Additional follow-up is also necessary to clarify the existence and nature of excess risks for myeloma and lymphoma.

\section{Cancer incidence}

The first comprehensive analyses of the LSS cancer incidence data were published in 1994. These data represent an important complement to the cancer mortality data. Despite the shorter follow-up period for incidence (since the tumor registries did not start operation until 1958), the number of cancer cases is greater than the number of deaths. The incidence data provide risk estimates for cancers with lower fatality rates, such as breast, thyroid, and skin, than most other cancers and help to clarify the picture for cancers like liver cancer for which death certificate information are often incomplete or inaccurate. One challenge facing RERF is the development of procedures that will allow the unification of the incidence and mortality data. The tumor registry incidence data already are being used as the basis for a number of site-specific incidence studies. In addition it is planned to make increased use of data on cancer incidence in the LSS as the basis for case-control studies.

For tumor registry data to be the basis for credible incidence studies, it is essential to maintain the present high quality standards of registry data, and close linkage must be maintained between the tumor registries and the LSS, $F_{1}$, and in-utero cohorts.

\section{Nonradiation risk factors}

Over the years, $A B C C / R E R F$ has conducted a number of mail surveys of members of the LSS cohort. These surveys, which provide some information on risk factors such as smoking, alcohol consumption, and occupation are an under-utilized resource. The mail survey data should be used to improve our understanding of radiation effects. In addition, case-control studies are used to examine the effect of nonradiation factors on the risk of cancer following radiation exposure. One example of such a study is a 
nested case-control study of liver cancer and hepatitis $C$ that is being carried out as a part of the liver cancer incidence study.

\section{Augmenting incidence studies with molecular biology}

The tissue registries and $A B C C / R E R F$ pathology programs are a valuable source of specimens and information for molecular oncologic and site-specific studies. Molecular studies are being developed to explore the genetic nature of radiation-induced breast cancer and, for example, the possible role of the $B R C A$ and/or $A T$ genes. The growing arsenal of genes implicated in carcinogenesis leaves little doubt that important targets for study will become apparent in the next few years. These studies currently conducted with the Department of Radiobiology may provide useful insights into radiosensitivity or mechanisms of radiation carcinogenesis. Molecular epidemiological investigations also may help to identify possible susceptible subgroups within a population.

\section{Substantiating noncancer mortality increases}

A recent noteworthy finding in the LSS is the evidence of a statistically significant increase in noncancer mortality as radiation dose increases. Lacking an experimental animal model for this effect, substantiation that this is a radiation effect requires careful investigation to eliminate the possibility that this effect is due to bias or confounding. Thus far, we have examined whether misclassification of cancer deaths as noncancer deaths might explain this result, and we have used Adult Health Study (AHS) data to look for evidence of radiation effects on specific endpoints related to heart disease and stroke. It was found that the levels of misclassification seen in comparisons of the RERF autopsy data with death certificate information cannot account for the noncancer effect. Although clinical monitoring of survivor health has revealed an association between radiation dose and heart disease or stroke and other risk factors, the findings to date should not be regarded as definitive. A comprehensive and more-unified epidemiological approach would enable further clarification of this controversial issue.

\section{Family studies}

Over the past 2 years, the RERF Scientific Council has supported the efforts to lay a foundation for family studies (genetic epidemiology). Thus far, limited efforts have been made to develop a family pedigree database for the LSS and $F_{1}$ cohorts. The BRCA and AT breast cancer studies noted earlier are prototypes in the development of a family studies program. To pursue this line of research, additional support in terms of RERF personnel and increased contact with specialists already active in this area are necessary. To successfully conduct genetic epidemiological studies requires resources (eg, computer and statistical expertise) and raises social and ethical issues that go 
beyond current RERF activities. Before proceeding in this area, all these issues must be considered.

\section{In utero studies}

Studies of the in-utero population (3289 persons) indicate that this group exhibits a broad range of health effects from radiation exposure and, in many respects, appears to be the most radiosensitive population. Radiation has marked effects on the developing fetus, including effects on the rate of growth and development, body size, and intelligence. Studies of the association between dose and severe mental retardation or IQ reductions of a less serious nature suggest that there are specific "windows of sensitivity" for these effects. Despite the relatively small size of the cohort and relatively small number of cancer cases to date, cancer risks do not appear to be dissimilar to those for LSS cohort members exposed as children. Evidence of excess noncancer mortality risk also exists in the in-utero cohort. The LSS data suggest that the excess risk is increasing as the in-utero cohort ages. It should be possible to obtain more insight into the effects of radiation on cancer and noncancer risks in this unique exposure group.

\section{Genetics studies}

An important part of the genetic studies is the ongoing epidemiological follow-up of mortality and cancer on over $80,000 \mathrm{~F}_{1}$ offspring (about $43 \%$ born to exposed parents and the rest to nonexposed parents). The follow-up has been based on both death-certificate-based mortality and tumor-registry-based cancer incidence.

As evidenced by the attention focused on the Sellafield (UK) leukemia cluster, issues related to the heritable effects of radiation exposure are of great public and scientific concern. The RERF first-generation $\left(F_{1}\right)$ cohort of children of the atomicbomb survivors is the only large-scale epidemiologic study able to address whether excess cancer or noncancer risks result from parental exposures. To date, no evidence of excess risks exists. However, cohort members are relatively young (with an average age of 39 in 1995), so several more decades of follow-up will be necessary to obtain a reasonable assessment of cancer risks (or the lack thereof) in this population. There is increased attention being devoted to this issue by imternational radiation protection programs, and the RERF prospective study will provide hard data independent of theoretical considerations.

\section{Clinical studies program}

\section{Brief description of the program}

The ABCC-RERF Adult Health Study (AHS) continues to be one of the largest and longest running clinical cohort studies in the world. During the 40 years of its existence, the AHS clinical program has played a key role in obtaining and sustaining support for ABCC-RERF from the survivors and the local communities. The biennial 
AHS medical examination is the only part of the RERF program that involves direct contact with survivors and thus serves as the main direct benefit that RERF provides the survivors. It is RERF's primary source of information for understanding noncancer effects of radiation. Through the AHS, biological materials are collected which will become increasingly useful for molecular and other biomarker studies of cancer and noncancer diseases. Again, it is stressed here that the effects of radiation exposure on diseases other than cancer remain largely unknown. The RERF clinical program is an irreplaceable source of biochemical and physiological measurements that underpin all of RERF's epidemiological analyses, including those of noncancer disease incidence.

Periodically, based on the follow-up of the AHS population, cross-sectional reviews of the status of health among the survivors have been published. The last review, $A H S$ Report 7, was the first comprehensive longitudinal analysis covering the period from inception in 1958 through 1986. This report was a milestone as pointed out by the Scientific Council because it was able to bring disparate studies into a coherent focus amenable to statistical analysis and demonstrated that the "enormous and valuable repository of serial medical data" is becoming an important research tool for epidemiological purposes. It demonstrated that the incidence of at least three diseases, myoma uteri (benign tumor), thyroid diseases, and chronic liver disease, significantly increases with increasing dose. Moreover, a number of serial laboratory measurements-blood pressure, cholesterol level, and calcium level-also show radiation-related shifts accentuating the changes seen in a normal aging population. AHS Report 8 is now in preparation, and we expect it to be even more comprehensive and informative than $A H S$ Report 7.

\section{Summary of the most recent results}

The initial clinical studies implied radiation effects on a variety of cardiovascular diseases (CVD), such as coronary heart disease (CHD), and stroke. Subsequent analyses in which confounding effects of known risk factors such as age, sex, hypertension, and smoking were taken into account indicated that the apparent radiation effects for CHD and stroke might reflect confounding. However, even after allowance for confounding by known risk factors a significant radiation effect on atherosclerosis is seen. This effect can be seen in a variety of endpoints: aortic arch calcification, measurements related to the coagulation cascade, isolated systolic hypertension, and retinal arteriosclerosis. As mentioned, increased noncancer mortality is notable among those exposed when young especially for coronary-related diseases. As this population reaches the later decades of life, much may be revealed about radiation effects on the young if follow-up can be maintained. Hyperparathyroidism among the atomic-bomb survivors is induced by radiation, and follow-up studies still in progress have provided further insights into this complex problem. Parathormone secretion increases the levels of serum calcium and alkaline phosphatase, leading to hypercalcemia and possibly enhanced vascular calcification. Radiation might cause the 
$\mathrm{C}$ cells of the thyroid to produce excess calcitonin which should have an antagonistic effect to parathormone but might be a feedback consequence of excess serum calcium. This web of relationships requires further unravelling.

\section{Present and future research directions}

As the AHS population moves into old age, the participation rate can be expected to fall dramatically. It is important to continue to obtain as much medical information relating to disease and health status of this study group as possible. To this end a health surveillance program has been introduced this year to follow up on the older age groups in the period between examinations. Telephone and mail requests for information on health status have obtained excellent response rates; moreover, those persons who have discontinued direct participation do provide information which would otherwise be unavailable.

One important ancillary role to the life span cancer study provided by the clinical study is determining what confounding factors influence cancer rates: for example, alcohol consumption, hepatitis- $\mathrm{C}$ virus infection and other factors which influence chronic liver disease and in turn hepatic cancer; smoking and lung cancer; and diet and colon cancer. A long-term study on proteinuria and benign monoclonal gammopathy confirms the observation of other groups that these may be precursor stages to the development of multiple myeloma, a malignancy not clearly demonstrated to be radiation-related. Continued monitoring should be informative because this is a condition that occurs increasingly with age.

The most important epidemiological role of the AHS program is monitoring of noncancer diseases that will subsequently result in mortality, such as atherosclerotic diseases and chronic liver diseases, the incidence of which seems to be increased by radiation exposure. New approaches to study these diseases are being introduced.

With a collection of 110,000 frozen samples of serum, it is possible to go back in time to determine if specific confounders, such as homocysteine levels (recently implicated in coronary disease), hepatitis $\mathrm{C}$ or other infections may have influenced the course of the above mentioned diseases. The clinical program also serves as the source of cell collection for the genetics, cytogenetics, and radiobiology studies and more recently for the collection of teeth for the ESR dosimetry analysis.

Listed below are several studies that are ongoing or should be started in the near future. Other projects also are described in departmental outlines supplied.earlier.

\section{Menopause}

On the basis of recall among female survivors, previous RERF clinical studies indicated that onset of menopause occurred earlier among the heavily exposed than among those who were not exposed or those who received low doses. Analysis of serum follicle-stimulating hormone (FSH) and estrogen levels in the 400 or so remaining premenopausal women is underway. This will permit a more definitive 
analysis of menopausal onset. Because postmenopausal women have higher cholesterol levels, this study also has bearing on cardiovascular disease issues, particularly since exposed women were the first group to show increased noncancer mortality. This study will near completion in 2 years.

\section{Senile dementia}

Another important study of the AHS population, which is midway to completion, is that of senile dementia in those over 60 years of age. Whether a radiation effect on the nervous system of persons exposed after birth exists remains to be seen. A similar dementia study is underway at the Honolulu Heart Center as part of the Ni-Hon-San collaborative program that compares the health status of persons of Japanese descent living in Hawaii, whose families came from Hiroshima, and participants of the AHS study here in Japan.

\section{Cataract}

Cataracts, primarily lenticular opacification, are radiation-induced, but RERF's last ophthalmological examinations were performed nearly 20 years ago. There is need to update this study. The US National Aeronautics and Space Administration (NASA) has already indicated an interest in developing a collaborative study, particularly because NASA studies indicate a late onset of serious cataracts in primates exposed when young. Those who were young at the time of the bombings now constitute the majority of our AHS population.

\section{Genetics program}

\section{Studies of the first generation born to atomic-bomb survivors}

The genetics program at RERF, a long-standing research project, is the singular study worldwide on the genetic effects of irradiation in humans. In 1991, the US National Academy of Sciences published a landmark book, The Children of Atomic Bomb Survivors. A Genetic Study. This volume is a compilation of all the major RERF papers produced over a 40 -year period.

It should be noted that the epidemiological study discussed earlier, as well as earlier studies on congenital defects, stillbirths, sex chromosome abnormalities and reciprocal translocations (on $16,000 F_{1}$ ), and the screening for protein alterations by onedimensional electrophoretic gels $\left(23,000 F_{1}\right)$ as well as by the enzyme activity measurements $\left(10,000 \mathrm{~F}_{1}\right)$, have not demonstrated a significant radiation-related increase in any endpoint. The combined mean parental dose is estimated to be $0.4 \mathrm{~Sv}$ (40 rem), with a majority of the children resulting from parents with exposures of less than $0.1 \mathrm{~Sv}$. Depending on various assumptions, the authors of the aforementioned studies have concluded that the genetic risk estimated by "doubling dose" procedures is higher by a factor ranging between 2 and 4 than the doubling dose of 1 Gy developed from mouse genetic studies. That is, the radiation dose needed to double the mutation 
rate is thought to be higher for humans, and thus humans may be 2 to 4 times less sensitive than the mouse. While the results to date show that the human genome is not unusually sensitive to irradiation and indeed may be less sensitive than that of the laboratory mouse, there are many reasons to believe that the mouse-human radiosensitivity differences are not conclusive. The doubling dose of 1 Gy developed from the mouse studies was based on specific locus mutations, ie, on known genes with definitive phenotypes. The major studies on untoward pregnancy outcomes are based on complexly inherited traits for which the genetic basis is uncertain, as implied in the assumptions. The doubling dose calculations for the mouse are predicated on a germ cell dose and mutation rate, whereas those for the human are based on a zygote dose and mutation rate. These methodological differences lead to a factor of 2 discrepancy. Clearly, further studies are expected to resolve the issue.

A few remaining genetic issues need to be addressed. The Human Germline Mutagenesis Workshop recommended the following: "Given the increased knowledge of genetic diseases, particularly polygenes/multifactorial diseases, further medical evaluations of the $F_{1}$ cohort should be considered." No systematic clinical examinations of these $F_{1}$ progeny have occurred since their first year of life. The majority of both dominant disorders and multifactorial inherited diseases are manifest after the first decade of life and, in the latter case, usually after adulthood. We would be remiss if we did not investigate the feasibility of undertaking at least a pilot study in this area. To this end we propose a workshop comprised of eminent genetic epidemiologists be convened to evaluate the prospects of a successful study in this area. Based on the very high participation rate, $75-80 \%$, of the children of the survivors during the period 1976-1984, when the cytogenetics and protein studies were undertaken, we can expect an enthusiastic response from them. There is every indication that the $F_{1}$ are anxious about possible health effects since media reports on other radiation-related studies continue to inflame their suspicions. This research project would of course be carried out within the clinical studies program, but it is discussed under future genetic studies since other background information is provided in this section.

\section{DNA studies}

At RERF, a major project to screen DNA samples from parents-child trios is underway. Establishment of cell lines from the trios was recommended by the Genetic Study Conference held in 1984. It is anticipated that immortalized B-lymphocyte cell lines from 1000 families, one-half of which are from exposed parents, will be maintained at RERF. Cell lines from 800 families composed of 1600 parents (with dose assignments) and 1200 children are now in our cell bank. This is the largest defined fixed cohort population in the world for the detection of radiation effects on human germline mutations. The Human Germline Mutagenesis Workshop held in 1991 recommended various targets and techniques. For various target loci, RERF 
scientists are now determining whether DNA deletions, insertions, rearrangements, or other quantitative changes, all commonly produced by radiation, exist in higher frequency in the "exposed" group. Major strides have been made in these technologies. These methodologies also improve upon the shortcomings of earlier approaches: Protein electrophoresis could only detect qualitative changes resulting from mutations producing single amino acid substitutions in the altered protein, and tests of enzyme activity measurements that could detect quantitative changes resulting mainly from deletion mutations were too few in number.

\section{Cytogenetic studies}

It is critical to the analysis of DNA alterations that the doses of the exposed parents be as accurate as possible. To this end, we believe that cytogenetic studies on the high-dose parents should be carried out as soon as possible to verify the derived dose estimates known to be prone to error for various reasons. Risk evaluations predicated upon the results of these molecular analyses depend on many factors including dose accuracy.

The cytogenetics unit of the Department of Genetics is now involved in biodosimetric studies of lymphocytes obtained from exposed AHS participants. New fluorescence in situ hybridization (FISH) techniques provide unbiased estimates of chromosome aberrations (reciprocal translocations) induced in these cells by the exposure 50 years earlier and which remain at relatively constant frequency throughout the life of the individual. The FISH approach and previous cytogenetic studies have indicated that physical dose assignments for those exposed in the open or in Japanese factories, for example, are often too high as compared to those exposed inside Japanese houses. This is particularly true for the Nagasaki dose estimates. It should be noted that the chromosome aberration frequency and the leukemia frequency both show similar linear-quadratic dose-response relationships, suggesting that some substantial fraction of the inducible leukemia types probably result from specific chromosome rearrangements.

Our recent study to compare chromosome aberration frequency with tooth enamel ESR demonstrated that the chromosome data correlated more closely to ESR data than DS86 estimates. The results not only confirmed the uncertainty associated with physical dose estimates but also provided the rationale to use chromosome data in place of DS86 estimates. Whereas teeth are not widely available, $2 \mathrm{ml}$ of blood is available for most of the AHS participants. The youngest cohort (ie, age ATB below 20 years) is known to show the highest relative risks for cancer, and nearly $90 \%$ of them are still alive. Thus, it appears an important task to perform cytogenetic tests on those AHS participants whose DS86 doses are above $0.6 \mathrm{~Gy}$, for example, so that the results may be used for future cytogenetic epidemiology. Such a study was not ever done in the world and is only possible at RERF. There are nearly 1500 people in total and it will take 5 to 7 years provided that the participation rate is about $70 \%$. 


\section{Radiation biology program}

\section{Introduction}

We believe that RERF's role in addressing the health effects of irradiation requires a comprehensive scientific attack on all the issues. Science, in general, and more recently biological science has the remarkable capacity to determine the basic mechanisms underlying the final observed health effect, be it cancer, heart disease, birth defect or other radiation-related diseases or conditions.

While epidemiology will provide the best answers to the questions of disease frequency and thus of overall health risks, as well as emphasize where studies are most fruitful, it can not answer the questions of mechanism nor can it be guaranteed that our basic research program will supply definitive answers to all these biological issues. It is, however, the case that through the combined efforts of our staff a wealth of biological material has been and will continue to be obtained from the survivor population, for whom an extensive health history is being maintained. We note that no other institution has the mandate, resources and commitment to continue this effort and important as they are, they are not more important than the existence of the survivors and their continued cooperation emphasizes this even more so. Funding cutbacks will only result in loss of this precious and irreplaceable resource. We have the added potential of establishing familial relationships among the survivors, since in a number of cases three generations are represented among the exposed groups. Molecular studies may reveal the existence of sensitive or cancer-prone subsets of the population if appropriate material is available.

If a specific constellation of genes is involved in the development of a specific cancer, it is now possible to detect which genes have mutated, more specifically which DNA changes and/or chromosome rearrangements resulted in that specific mutation and which mutation was radiation-induced (if most of these are gross DNA alteration of the gene). Why are certain cancers radiation inducible and others not? Why do certain cancers develop rapidly after exposure, whereas others require half a lifetime before they are expressed? What role does the immune system play in the progression of aging and ill health? Are induced changes in specific immune cell subsets likely to affect the manifestation of specific diseases through a change in susceptibility? These are among the many questions our radiobiology program is either engaged in/or poised to address, as will be discussed.

\section{Molecular oncological approaches}

The molecular biological and oncological approaches consist of the analysis of tumor and normal tissues from the same individuals to determine which mutation steps in the process of malignancy might be radiation-induced. It is thought that radiationinduced events might differ qualitatively and quantitatively from the mutations that arise from nonexposure conditions. The ongoing studies in this area involve skin, 
breast, liver, thyroid, and lung. About 12 known oncogenes or tumor suppressor genes are being examined. Remarkably, this research group has succeeded in amplifying gene regions from archival autopsy material that is over 40 years old with results similar to those obtained from fresh surgical material. It should be noted that our data on early onset (before age 35) of breast cancer in women exposed as children has suggested that a heritable mutation also may be involved. If RERF's archival repositories include some of these specimens, the presence of $B R C A 1, B R C A 2$, or AT mutations in both normal and malignant cells might be demonstrated. These studies are in progress and have begun over the last $1-3$ years.

\section{Immunological approaches}

The functionality of the lymphocyte system is a key measure of the ability of atomic-bomb survivors to respond to infectious disease. Abnormalities in this system also lead to carcinogenesis and autoimmune diseases. Aging and radiation exposure affect immune competence, altering T-cell subsets both in their number and in their differential responsiveness when challenged by various stimuli. Therefore, study of the immune system has been critical to assessing the radiation-induced effects that might affect the long-term health of the survivors.

Technically possible only within the last several years, the study of the immune system repertoire will likely reveal temporal changes in the immune function of individuals. This is especially true for those who were young at the time of the bombings (ATB) and exposed to high doses. RERF's multidimensional research environment allows the correlation of immune system changes with the morbidity and mortality data of the AHS cohort.

Researchers in this program have begun to focus attention upon the recovery of hematopoietic progenitor cells (ie, stem cells) to better understand radiation-induced alterations of hematopoietic function.

\section{Somatic mutation studies}

Immunological assays either developed at RERF or adapted to RERF's special needs are used to measure somatic mutations in the atomic-bomb survivors and other more recently exposed populations, such as Chernobyl accident-affected persons.

Lymphocyte cells from survivors have been studied for induced somatic mutation at three different gene loci: $H L A, T C R$ and HPRT. These studies have indicated that the lymphocyte system is a poor monitor of exposure occurring many years earlier, probably because such mutations exert a deleterious effect on cell function and viability. However, these study systems have proved quite useful in studies of individuals who received recent acute radiation exposures. The persistence of such mutant cells parallels that found for unstable chromosome aberrations induced in lymphocytes. 
In contrast, RERF studies of the erythrocyte glycophorin A (GPA) mutation have revealed a linear dose response in more than 1000 atomic-bomb survivors. The usefulness of this assay as a population biodosimeter is now clearly established.

We would like to make special note of the fact that the linear dose response for GPA somatic mutations is similar to the dose response observed for solid tumor incidence among the atomic-bomb survivors. Since carcinogenesis is a multistage developmental process usually affecting adults in middle age or beyond, the Department of Statistics is incorporating this data into new models to formulate how the induced events might accelerate the onset of malignancy.

Because mature red blood cells lack a nucleus, molecular analysis to characterize radiation-induced mutations in their DNA is not possible. However, using reticulocytes obtained from 10 high-dose survivors, RERF researchers have demonstrated that GPA mutations were most probably deletions derived from the pluripotent stem cells of the bone marrow. By continuing this reticulocyte study, a more-definitive characterization of induced mutations in this cohort will be attempted in the future.

If the research breakthroughs of the last decade in areas of molecular biology and immunology, for example, are forerunners of what may be expected over the next decade, then there can be little doubt that future advances will allow us to utilize our unique biological repositories to answer questions still unformulated. Who would have predicted even 10 years ago that specimens archived 40 years ago could be reanalyzed with PCR and molecular sequencing techniques, or that chromosomes could be painted so that rearrangements could be rapidly detected, saving years of effort. We are well prepared to take advantage of such future developments.

\section{Repository of biological materials}

An extremely valuable asset of this 50 -year program is the continuing maintenance of biological specimens. The Life Span Study has 7500 autopsied specimens and 13,000 surgical specimens collected mainly in the early years. Considerable effort is underway to expand the collection of more-current material.

The clinical program maintains a collection of 110,000 frozen serum samples and 11,000 plasma samples spanning the last 30 years of the program. In addition we have collected 13,000 lymphocyte samples for the Department of Radiobiology, which maintains this collection in liquid nitrogen. This collection represents one or more samples from about one-half of all the surviving AHS population. The clinical program has obtained all the 800 family trio lymphocyte samples for the biochemical genetics program. The genetics group then maintains both $\mathrm{EB}$ virus-transformed and -untransformed lymphocytes in liquid nitrogen. Cryopreserved lymphocytes are capable of subsequent reproduction. 


\section{Data management}

Great progress has been made during the past 3-4 years in establishing a relational database system capable of integrating data from the different RERF research programs. The database has eliminated data redundancy and greatly improved data retrieval and linkage capabilities. All persons of interest to RERF, including the three major cohorts and those who participated in ABCC studies before the establishment of the Unified Study Program (1955) are registered in the database. The Master File, tumor registries, tissue registries, Life Span Study, and DS86 data are included and are linked to the appropriate individuals. Follow-up data for all studies are included. Despite this progress, much of the ABCC/RERF data remain stored as independent files and need to be added to provide more complete information on individuals in the study cohorts. Progress on much of this work will depend on the availability of experienced database management personnel and appropriate computing resources.

It is important to provide our researchers with online access to documentation of the RERF database, as well as training on linkage and retrieval methods. Work on these aspects has begun. Efforts to clarify the definitions and data sources for the $F_{1}$ and in utero cohorts are nearing completion, after which this information will be added to the database.

In anticipation of future revisions to the DS86 dosimetry, it will be important to incorporate all individual shielding history information into the database, and to clarify the exposure-related data available for these individuals. These efforts also will help identify additional persons for whom enough data is available to permit calculation of DS86 estimates. This has important bearing on the $F_{1}$ and in utero studies, where excluding participants from analyses due to the absence of parental dose estimates greatly affects the statistical power of analyses due to small sample sizes and/or relatively small numbers of cases.

The biological materials collected and stored at RERF represent a valuable resource for current and future studies. Inventory records have been managed separately by the research programs maintaining the samples. To ensure efficient use of these materials, information on the existence and nature of these materials will be added to the RERF database and linked to the study participant donors.

Plans are under design to add other information, such as longitudinal clinical data from the AHS and data from surveys conducted over the years, to facilitate information linkage across program disciplines. This capability will serve as a particularly important tool in more-comprehensive approaches to analyses of morbidity and noncancer mortality.

As molecular epidemiologic and genetic studies progress, it is anticipated that additional data management support will become necessary to handle the enormous amount of data that will be generated by these studies. 


\section{Atomic-bomb dosimetry}

Individual physical dose estimates are a core component of the RERF research program. In the years since the introduction of Dosimetry System 1986 (DS86), concerns about systematic errors continue to be voiced. In particular, thermal neutron measurements obtained from exposed building materials suggest that DS86 neutron doses in Hiroshima at distances beyond about $1000 \mathrm{~m}$ from the hypocenter may be too low by a distance-dependent factor ranging up to 10 . While it seems unlikely that such changes in neutron doses will have a major impact on risk estimates, rapid resolution of this issue is desired. Since decisions about the nature of the dosimetry system are made outside RERF, it would be useful if the relevant groups could provide RERF as soon as possible with either a revised dosimetry or with guidelines for adjustment of current estimates to account for the most probable changes. Another important issue related to DS86 concerns dose estimates for Nagasaki factory workers. LSS cancer mortality data and biodosimetric data suggest that DS86 dose estimates for factory workers are too high. Since factory workers are a large fraction of the heavily exposed survivors in Nagasaki, systematic errors in their dose estimates can have a major impact on risk estimates. Again, some guidance from external groups concerned with the survivor dosimetry would be useful in determining how to handle this issue.

The availability of physical dose estimates for a large population with a broad range of exposures has made RERF the most important center for developing and assessing long-term biodosimeters. Identifying stable chromosome aberrations in lymphocytes has long been the mainstay of biodosimetric work at RERF.

The glycophorin A (GPA) assay discussed earlier is a second biodosimeter that correlates with dose. However, because of the relatively wide variability in mutation frequency it is more useful in determining population exposure levels than in characterizing individual dose estimates. The development of new somatic mutation assays should improve the accuracy of dosimetry estimates.

Another dose verification method is being pursued at RERF. Preliminary electron spin resonance (ESR) studies using tooth enamel of survivors indicate that the physical dose estimates obtained by reading this "personal film badge" correlate far more closely with chromosome aberration frequencies from the same individuals than the DS86 dose assignments. The excellent agreement between the two dosimetry methods, a biological and a physical one, must mean that they are correlating to the true dose and that the DS86 dose estimates reflect the impact of random errors on DS86 estimates. Moreover, the ESR approach seems to have clarified why persons assigned the same DS86 dose have reported different degrees of epilation. Rather than evidence of a variation in radiosensitivity (as some have suggested), it is further evidence of errors in DS86 dose assignments, because the ESR measurements (and chromosome data) are much higher for epilators than for nonepilators. 


\section{Collaborative programs}

The results of RERF studies continue to have a major impact on the understanding of radiation risks and the establishment of standards for radiation protection. RERF scientists are actively involved with the International Commission on Radiological Protection (ICRP), the United Nations Scientific Committee on the Effects of Atomic Radiation (UNSCEAR), the US National Council on Radiation Protection, the US National Academy of Sciences Advisory Committee on the Biological Effects of Ionizing Radiation, and other groups in the interpretation of RERF results. After the Chernobyl accident in 1986, RERF has become more directly involved in studies of radiation effects in other populations whose qualitative exposures differ markedly from the atomic-bomb survivors. Many of these activities are being carried out in conjunction with other groups, including the World Health Organization (WHO), the International Atomic Energy Agency (IAEA), and Gesellschaft für Strahlung Forschung (GSF). RERF recently has contracted with the US National Cancer Institute (NCI) to provide direct support for efforts to strengthen the epidemiologic studies of workers and members of the general population exposed to radiation as a consequence of plutonium production in the southern Ural Mountains of the Russian Federation.

RERF is actively cooperating with the local organizations in Hiroshima (Hiroshima International Council for Health Care of the Radiation-exposed-HICARE) and Nagasaki (Nagasaki Association for Hibakusha's Medical Care-NASHIM). These organizations provide advisory medical and technical personnel to countries where major radiation accidents have occurred. In addition HICARE and NASHIM have provided funds for staff from these countries to receive specialized training in Japan. It is anticipated that RERF scientists will become increasingly active in such collaborative efforts.

In the connection with the foregoing, we wish to draw your attention to the resolution which was made at the 27th meeting of the RERF Board held in Hiroshima in June 1993. The resolution reads as follows:

"Whereas the Radiation Effects Research Foundation (RERF) has been overwhelmed in recent years by physicians and scientists from various parts of the world (eg 336 visitors in 1992), all seeking advice on radiation effects research programs, the Board of Directors at $R E R F$ has resolved that the United Nations should be requested to establish and support at RERF the (United Nations) International Radiation Health Effects Advisory Center. WHO, IAEA, UNSCEAR, as well as the governments of Japan and the US, should be advised of this request and be asked to support it."

RERF has been designated as a WHO Collaborating Centre for Radiation Effects on Humans since 24 May 1979 and also a member of the WHO's Radiation Emergency Medical Preparedness and Assistance Network (REMPAN) since October 1988. 
However, many trainees and visitors come to RERF not only from WHO but also from IAEA and other UN-related agencies. This is the reason why our Board of Directors considered that a new training center sponsored by UN should be established at RERF.

We note that all prospective research projects involving our study population, collaborative or not, are subjected to a rigorous review process before executive approval to initiate the project occurs. In any collaborative study it is RERF's responsibility to maintain in strict confidence the identity of all atomic-bomb survivors. At present there are 31 active research projects which include physicians and scientists from other medical and research centers, usually in Hiroshima and Nagasaki. RERF actively seeks out these collaborations. Unfortunately, few if any requests from within Japan to undertake research of mutual interest have occurred (except to request biological specimens).

For 5 decades, James V Neel, University of Michigan, and William J Schull, University of Texas, have provided guidance and training for scientists in our genetics program. Most recently technical and analytical methodologies in DNA biochemical genetics have been developed in collaboration with the Human Genetics Department at the University of Michigan. Dr Schull's interaction with scientists from the departments of Statistics and Epidemiology has resulted in publications on reanalysis of the in utero-exposed mental retardation and IQ data based on DS86. We also have maintained a strong collaborative program in epidemiology with Charles $E$ Land and Elaine Ron of the cancer epidemiology section of the US National Institutes of Health (NIH). NIH has provided financial support for specific projects. Most recently three studies on breast cancer have resulted from this collaboration, and a manuscript on salivary gland cancer has been completed. For 20 years our clinical program has collaborated with the Honolulu Heart Center, as described elsewhere in our documents. Two recent publications with other research branches of the NIH involve p53 gene mutations and lung cancer, and the role of JC polyoma virus in producing unusual chromosome aberrations in somatic cells. A pilot study between the University of Leiden and the Department of Radiobiology to screen for radioresistant lymphocytes has just begun.

It may also be noteworthy to mention that the Japanese Ministry of Health and Welfare has been providing a special research grant of about 20 million yen every year since 1981 to the Chairman of RERF. The purpose is to promote the collaborative studies on atomic-bomb survivors between RERF and other research institutions mainly in Hiroshima and Nagasaki.

For 10 years RERF supported 1-year traineeships for Chinese researchers who studied here. For many more years, RERF awarded two summer research fellowships to medical students from the University of Rochester. Both these programs were discontinued recently because of lack of funds. Extramural funds support training at RERF of scientists from the former USSR in cytogenetics, radiobiology, and biostatistics. 
Finally, we would like to add that the Hiroshima Prefecture is now planning to establish a prefectural cancer center with emphasis on radiation carcinogenesis research and RERF has been requested to cooperate with them in this plan.

\section{Maintaining competent research staff}

In order to continue RERF's core research programs as well as the collaborative studies, recruiting and maintaining a strong and motivated scientific staff is a pressing concern. Recruitment of professional staff occurs in general at two levels: the permanent long-term employment of Japanese researchers in the areas of greatest need and the generally shorter term employment of foreign staff through the US National Academy of Sciences.

Recruitment is a high priority in epidemiology to support the following endeavors in particular: developing a more comprehensive and systematic approach to investigating radiation effects on noncancer mortality; planning and coordinating genetic and molecular studies; and ensuring continued high-quality management of RERF's broad range of epidemiologic studies. In view of recent nonreplacement of staff prompted by current fiscal constraints, the addition of two or even three epidemiologists with the appropriate interests and abilities would not be unreasonable and would benefit all of RERF. Staffing shortages in computing and statistics are a recurring problem that adversely affects epidemiology projects as well as work Foundationwide. Given the nearly 50 years of heavy financial support provided by the US to this program, it is indeed remarkable that the US presence at RERF is now reduced to only 10 staff members, of whom only 5 are on direct research programs and another 2 are engaged in computer management activities. US staff support to the epidemiology and allied programs is sorely needed. These are the areas in which US expertise is strong and in which the mounting data from the Life Span Study and Adult Health Study programs require effective analysis.

\section{Summary}

The main epidemiologic follow-up investigations include the Life Span Study, the Adult Health Study, and the studies of the survivors' children. About 50\% of the populations included in the first two studies, which provide mortality and morbidity data, are still alive. Moreover $90 \%$ of those exposed in childhood are alive. These studies focus on the central questions of whether radiation-induced excesses can be observed in these data and whether risk estimates change over time. The influence of confounding factors and synergisms with other environmental or biological factors are being explored through mail and interview surveys and case-control studies. Verification of cancer incidence data hinges upon the tumor and tissue registries and the histological work done under the aegis of the epidemiology program. The data management group, involving several departments, is striving to update and merge 
disparate data files to facilitate comprehensive data analysis. Only partly achieved to date, this data consolidation is an ongoing project.

The Adult Health Study projects are designed to identify radiation-induced changes in the survivors reflected in a change of health status that may or may not be the ultimate cause of death, but certainly can affect the quality of life. These studies include cataract, menopause, senile dementia, and other systemic changes that impact on overall homeostatic mechanisms. Moreover, the in-depth longitudinal health studies of this program are unequaled by any other study group, and sophisticated statistical procedures only recently have been developed to tap this wealth of information.

A well-designed clinical study of the $F_{1}$ cohort using currently available screening methods to identify better defined sentinel phenotypes could provide useful information about genetically based detriment to health and a workshop to determine the feasibility of such a study is recommended.

As discussed, our major ongoing molecular study in genetics can be expected to determine whether induced mutations were transmitted to the progeny at a variety of genetic sites and what the molecular nature of such changes are. The concurrent cytogenetic study of the parents' lymphocytes is recommended to substantiate DS86 dose assignments.

Radiobiology projects include ongoing analytical studies on the nature of molecular changes involved in tumor development; the development of somatic mutation assay systems for biodosimetric purposes; and a range of studies attempting to understand any radiation-induced molecular changes in the human immune system that might be reflected in the health of the survivors.

Biological materials obtained from survivors are scrutinized for evidence of somatic mutation measured by both gene and chromosomal changes which serve as biological dosimeters. Results have highlighted areas of the physical dosimetry that need to be reassessed.

RERF interacts with every major binational and international program concerned with radiation health effects either directly through staff representation in these programs or by supplying our most recent data for incorporation into their review documents. Our studies continue to be the core of all risk analyses undertaken worldwide. In this connection RERF should play a larger role as an international training center on radiation health studies.

RERF has responded to the continuing budget crisis and the requirement of no staff replacement by eliminating nonessential or nonmission-oriented programs and transferring personnel to other key areas. The result has been a decrease in general and professional staff. However, if the policy continues for 2 more years, there will be 37 additional mandatory retirements (and approximately 20 voluntary retirements). Nearly $60 \%$ of these will occur in the epidemiology and clinical studies programs, involving primarily highly trained technical staff not replaceable through internal 
transfers. We cannot continue to sustain these reductions and remain a productive research program.

Because RERF's focus is research, staff members are only informally engaged in efforts to educate the lay public about RERF's mission and achievements. Except for occasional cursory newspaper articles about RERF's annual scientific meetings, the local media do not eagerly cover the often complex scientific topics that are most noteworthy. Nonetheless, public interest in RERF is high, as evidenced by the first public openhouse of the Hiroshima Laboratory held this year on 5 August, Saturday before the 50th anniversary of the atomic bombing. More than 900 persons of all ages, wandered freely through the facilities, visiting scientific laboratories and supportservice offices. Scientists, particularly in the labs and clinic, along with their support staffs, fielded questions for hours. Clearly, the community is keenly interested in RERF's work.

Five decades after the bombings, many hibakusha (Japanese for "atomic-bombaffected persons") still rely greatly on RERF research and health examinations, just as they did during the time of the Atomic Bomb Casualty Commission (1948-1975). Quoted from the ABCC Annual Report, 1966-67, the director of a survivors' organization, when visiting with $\mathrm{ABCC}$ Director George B Darling, stated: "We all know that the purpose of $A B C C$ is research. We believe in it. We support you." 


\section{Major Meetings}

\section{2nd Scientific Council Meeting}

The 22nd Scientific Council meeting was held at the Hiroshima Laboratory for three days from 19 to 21 April 1995.

Chairman Itsuzo Shigematsu gave an account of the negotiations between the Ministry of Health and Welfare (MHW) and the Department of Energy (DOE) concerning the budget and operations of RERF. Dr Harkness, chief of research, in his report, emphasized that research is being conducted at the same level as in the past and that the number of papers presented remains unchanged though the number of staff members has been reduced due to a drastic budget cut. Subsequently, the salient points of the studies and the results produced were reported by each department. The studies reported received high marks.

The employees and directors of RERF as well as researchers in Japan and abroad expressed their concern about the DOE's announcement of changing the US overseer of RERF from the US National Academy of Sciences (NAS), which has been involved in the management of RERF for many years, to some universities in the United States.

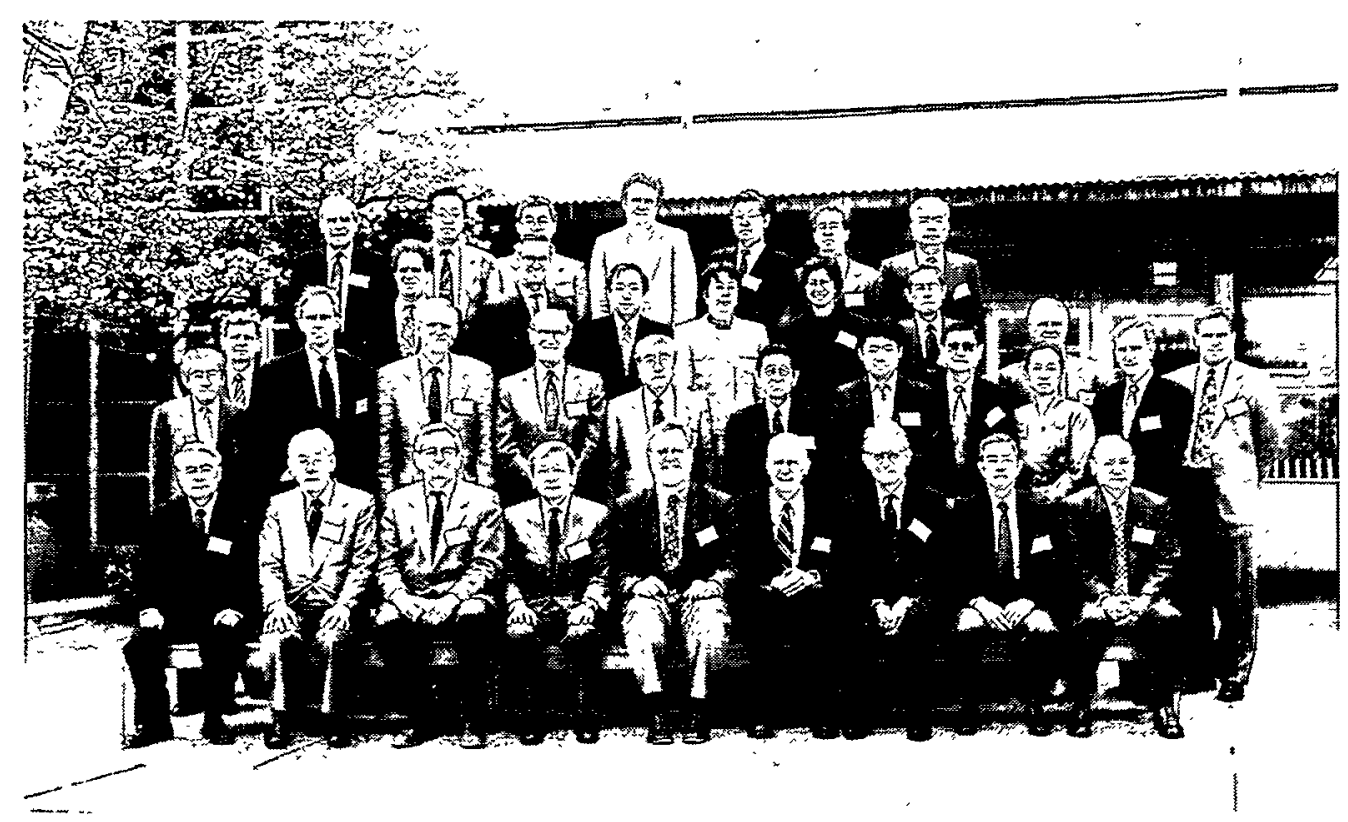

Attendees of the 22nd RERF Scientific Council meeting included, front row from left, S Okada, E Matsunaga, CW Heath Jr, E Ishikawa, CC Harris, JB Little, AG Motulsky, $H$ Matsudaira, $T$ Hirohata. Second row from left, $T$ Kumatori, RD Sperry, CJ Huyskens, CE Land, ML Mendelsohn, I Shigematsu, A Shishido, H Maruyama, JD Zimbrick, $R$ Maeshiro, $M$ Eaton, $P$ Gilman, TS Fox. Third row from left, $S$ Galson, $J$ Mulvihill, M Akiyama, $C$ Satoh, JL Ohara, $Y$ Hasegawa. Back row from left, DR Harkness, K Kodama, Y Shibata, DL Preston, AA Awa, K Mabuchi, Y Hirano. 
As a measure to resolve the issue, the Scientific Council recommended that an international committee of distinguished scientists be appointed to make recommendations on the RERF's future scientific activities. The council also suggested that DOE defer its plan of changing the US overseer of RERF until the international committee submits its recommendations.

Summarized minutes and detailed Scientific Council recommendations begin on page 145 of the Appendix.

\section{Blue Ribbon Review Committee Meeting}

A committee of internationally prominent experts (so-called Blue Ribbon Panel) was established on the recommendation of the Scientific Council at its 22nd meeting held in April 1995 to review the future of RERF research activities. This panel held its first meeting at the Hiroshima Laboratory and the Nagasaki Laboratory from 4 to 8 February 1996 to make preliminary arrangements and to hear overviews of RERF's research and support activities.

This was for addressing the various problems facing the RERF; namely, the reduction of its budget due to the difficult financial situation of the United States continuing from the previous year, the budget reduction measure taken on account of the unfavorable change in the exchange rate of the dollar, and the proposal made by the US Department of Energy (DOE) in January 1995 to change the US side's RERF oversight organization from the US National Academy of Sciences (NAS) to a university in the United States.

The recommendation of the Scientific Council was approved at the Board of Directors meeting held in June 1995 and, after its approval by the governments of Japan and the United States, the Blue Ribbon Panel was established in September the same year. Cancellation of the contract with NAS, which the DOE had proposed, was shelved in consequence of the binational agreement to establish the Blue Ribbon Panel, and the contract was extended for two years. After further meetings, the panel will compile and submit its final report to the governments of Japan and the United States in July 1996.

The members of the Blue Ribbon Panel are as follows.

\section{Membership of the Blue Ribbon Panel}

Chairman

Roger $\mathbf{H}$ Clarke, director, UK National Radiological Protection Board and chairman of Main Commission, International Commission on Radiological Protection

\section{Members}

Tadao Shimao, president, Japan Anti-tuberculosis Association; Japanese chairman, US-Japan Medical Cooperation Committee 
Wataru Mori, president, Japanese Association of Medical Sciences; member, the Council for Science and Technology; and former president, Tokyo University

Jack H Geiger (United States), chairman, Department of Community Health and Social Medicine, City University of New York Medical School

Herbert L Abrams (United States), professor of radiology, Department of Radiology, Stanford University School of Medicine

Dan Beninson (Argentina-recommended by Japan), president of the board of directors, National Board of Nuclear Regulation; member (former chairman) of Main Commission and chairman of Committee 4, International Commission on Radiological Protection

Keith H Lokan (Australia-recommended by Japan), director, Australian Radiation Laboratory and member of Committee 4, International Commission on Radiological Protection

Valerie Beral (United Kingdom-recommended by the United States), director, Imperial Cancer Research Fund, Cancer Epidemiology Unit

Albrecht Kellerer (Germany-recommended by the United States), direktor des Strahlenbiologisches Institut, Ludwig-Maximilans-Universität

\section{Executive Secretary}

Colin R Muirhead, UK National Radiological Protection Board (technical assistant to the Panel)

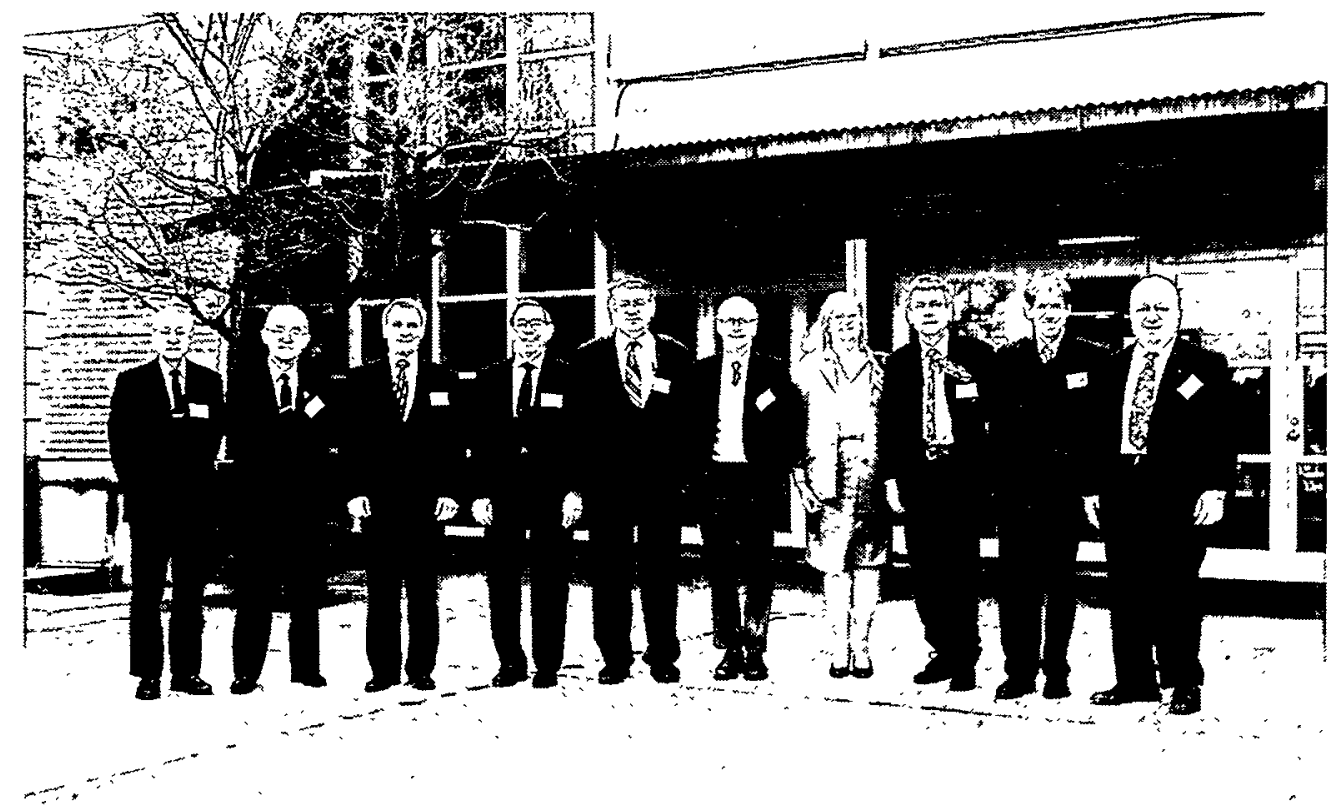

Members of the Blue Ribbon Panel: from left, Wataru Mori, Tadao Shimao, Roger $H$ Clarke, Jack H Geiger, Keith H Lokan, Herbert L Abrams, Valerie Beral, Albrecht Kellerer, Colin R Muirhead, and Dan Beninson. 


\section{Abstracts of RERF Reports Published in Journals}

\section{April 1995-31 March 1996}

\section{RERF Reports}

Editor's note: RERF Reports begin as manuscripts that are officially approved by the RERF permanent directors for submission to journals. Upon their acceptance by a journal, such manuscripts are designated as RERF Reports. RERF Reports consist of journal-article reprints that are purchased from the publishers and bound in RERF Report covers with a Japanese summary. When the report is followed by a $(\mathrm{J})$, a Japanese version of the report will be available. Variation in summary text styles reflects different journal styles.

RERF Report No. 23-93 Cytogenetic and immunologic identification of clonal expansion of stem cells into $T$ and $B$ lymphocytes in one atomic-bomb survivor. Y Kusunoki, Y Kodama, Y Hirai, S Kyoizumi, N Nakamura, M Akiyama. (Published in Blood 86:2106-12, 1995.)

Chromosome aberration frequency in peripheral blood lymphocytes is elevated in radiation-exposed people, and identical karyotypic changes are not infrequently encountered in one blood sample as well as in separate samples from the same donor. Such clonal propagation originates either from a single immature stem cell able to expand and differentiate into several cell types or from a single mature lymphocyte able to expand after antigen stimulation in vivo. In the present study, a total $71 \mathrm{~T}$ lymphocyte and 58 B-lymphocyte colonies were established from one atomic-bomb survivor, who showed a persistent clonal aberration $t(4 ; 6), t(5 ; 13)$ in phytohemagglutinin culture of peripheral lymphocytes. Nearly $10 \%$ of the colonies $(6$ T-lymphocyte and 7 B-lymphocyte colonies) showed the same chromosome abnormality. Southern blot analyses of the T-cell-receptor or Ig heavy-chain gene showed all different rearrangement patterns among $\mathrm{T}$ - or B-lymphocyte colonies, respectively. Thus, the chromosome aberration occurred in a precursor cell before differentiation into $\mathrm{T}$ and $\mathrm{B}$ lineages and was not derived from monoclonal proliferation of mature $\mathrm{T}$ or $\mathrm{B}$ lymphocytes in the periphery. To confirm the issue, cells from erythroid burst-forming unit (BFU-E) colonies were examined by the chromosome-painting method. Two translocations, one between chromosomes 5 and 13 and the other between chromosomes 4 and one of group C, perfectly consistent with the $t(4 ; 6), t(5 ; 13)$, were found in about $10 \%$ of the cells. The results imply that a single stem cell of an adult is capable of generating long-lived myeloid and lymphoid progeny 
amounting to several percent of the total population of circulating lymphocytes and hematopoietic progenitors.

RERF Report No. 28-93 Optimal case-control matching in practice. JB Cologne, Y Shibata. (Published in Epidemiol 6:271-5, 1995.)

We illustrate modern matching techniques and discuss practical issues in defining the closeness of matching for retrospective case-control designs (in which the pool of subjects already exists when the study commences). We empirically compare matching on a balancing score, analogous to the propensity score for treated/control matching, with matching on a weighted distance measure. Although both methods in principle produce balance between cases and controls in the marginal distributions of the matching covariates, the weighted distance measure provides better balance in practice because the balancing score can be poorly estimated. We emphasize the use of optimal matching based on efficient network algorithms. An illustration is based on the design of a case-control study of hepatitis B virus infection as a possible confounder and/or effect modifier of radiation-related primary liver cancer in atomic bomb survivors.

RERF Report No. 4-94 Vertebral fracture prevalence in women in Hiroshima compared to Caucasians or Japanese in the US. PD Ross, S Fujiwara, C Huang, JW Davis, RS Epstein, RD Wasnich, K Kodama, LJ Melton. (Published in Int $J$ Epidemiol 24:1171-7, 1995.) (J)

Background. Although vertebral fractures are very common among elderly Caucasian women, no studies have compared the prevalence to that among Asian populations. Any observed differences in prevalence might lead to the identification of important environmental and/or genetic factors. We therefore compared the prevalence of vertebral fractures among US Caucasians to native Japanese and Japanese immigrants in Hawaii using a standardized approach.

Methods. Spinal radiographs of women aged $\geq 50$ years were obtained from native Japanese in Hiroshima, Japanese-Americans in Hawaii, and North American Caucasians in Minnesota between 1982 and 1991. Fractures were defined as vertebral heights $>3$ standard deviations (SD) below the vertebra-specific mean.

Results. Compared to Japanese-Americans, odds ratios (OR) and $95 \%$ confidence intervals (CI) for prevalent vertebral fractures were 1.8 (95\% CI: $1.3-2.5$ ) for native Japanese women and 1.5 (95\% CI: 1.1-2.1) for Minnesota Caucasians. The OR tended to be higher when comparing the prevalence of two or more fractures per person: $\mathrm{OR}=$ 3.2 (95\% CI: $2.0-5.3$ ) for native Japanese and OR $=1.9$ (95\% CI: 1.2-3.2) for Minnesota Caucasians. Similar results were observed for native Japanese using a fracture definition of $\geq 4$ SD below the mean, but the OR for Caucasians was reduced to 1.2 (95\% CI: 0.6-2.3). 
Conclusion. The observation that, among these three populations, hip fracture incidence is lowest but spine fracture prevalence is greatest among native Japanese suggests that different risk factors may be responsible.

RERF Report No. 7-94 Somatic-cell mutations as a possible predictor of cancer risk. M Akiyama, S Umeki, Y Kusunoki, S Kyoizumi, N Nakamura, T Mori, Y Ishikawa, M Yamakido, K Ohama, T Kodama, K Endoh, JB Cologne. (Published in Health Phys 68:643-9, 1995.)

The somatic-mutation theory of carcinogenesis has received strong scientific support from results of recent studies on tumor-suppressor genes. We anticipated that people among the high risk for cancer group, either through exposure to various ionizing radiations or by virtue of unique genotypes, would also manifest increased frequencies of somatic mutation. This report presents the results of two somatic-mutation assays-at the erythrocyte glycophorin A (GPA) and lymphocyte T-cell receptor (TCR) genes-in various groups at high risk for cancer development, including atomic-bomb survivors, patients with various cancers, patients administered Thorotrast, and patients with genetic disorders that make them cancer prone. Although neither the GPA-mutation nor the TCR-mutation assay detects gene mutations directly related to carcinogenesis, increased mutation frequencies were detected by both assays in many individuals among the high-risk groups and among cancer patients. We have continued to follow up those individuals who show values of about three times higher than those of the control group. Thus, these assays may prove useful for identifying high-risk cancer groups and for estimating the effects of mutagens. Such information would constitute a valuable data base for epidemiological studies.

RERF Report No. 12-94 Effect of radiation and cigarette smoking on expression of FUdR-inducible common fragile sites in human peripheral lymphocytes. S Ban, JB Cologne, K Neriishi. (Published in Mutat Res 334:197-203, 1995.)

In vitro $\mathrm{X}$-irradiation of human peripheral blood lymphocytes increased the frequencies of fluorodeoxyuridine-induced fragile sites in a dose-related manner. However, the cells from 30 atomic bomb survivors exposed to either high or low radiation doses 47 years earlier showed no demonstrable difference in fragile site expression, indicating that fragile site induction was ephemeral in nature. When fragile sites were analyzed on the basis of tobacco smoking habits, an elevated number was observed in the smokers. The results confirm that fragile sites can be affected by recent exposure to exogenous agents, but the effect is probably of limited duration, based on the atomic bomb survivor experience. 
RERF Report No. 13-94 Mutant frequency at the HPRT locus in peripheral blood T-lymphocytes of atomic bomb survivors. Y Hirai, Y Kusunoki, S Kyoizumi, AA Awa, DJ Pawel, N Nakamura, M Akiyama. (Published in Mutat Res 329:183-96, 1995.)

The mutant frequency at the hypoxanthine-guanine phosphoribosyltransferase locus in peripheral blood lymphocytes was measured for 254 atomic bomb survivors (171 exposed and 83 control survivors) by a colony assay using recombinant human interleukin-2. Weak but significant effects were detected for atomic bomb radiation dose and smoking status at the time of examination but not for age and sex. However, the slope of the dose-response curve is quite small, and the smoking effect would not have been significant without the inclusion of data from just three individuals with extremely high mutant frequencies. The weakness of the dose response is at least partly due to the time lapse of 50 years since radiation exposure. Among the 254 survivors, 23 had chromosome aberration data in lymphocytes and the dose response was highly significant. However, the correlation between the mutant frequency and the proportion of cells with aberrations was not significant. It was concluded that the lymphocyte mutation assay is presently not sensitive enough for biological dosimetry of radiation exposure in the survivors.

RERF Report No. 14-94 Anti-human T-Iymphotropic virus type-I antibodies in atomic-bomb survivors. T Matsuo, E Nakashima, RL Carter, $\mathrm{K}$ Neriishi, $\mathrm{K}$ Mabuchi, M Akiyama, K Shimaoka, K Kinoshita, M Tomonaga, M Ichimaru. (Published in J Radiat Res [Tokyo] 36:8-16, 1995.)

Adult T-cell leukemia (ATL), induced by human T-lymphotropic virus type-I (HTLV-I), is endemic in Nagasaki, Japan. To investigate the effects of atomic-bomb radiation on development of this specific type of leukemia, 6182 individuals in the Radiation Effects Research Foundation (RERF) Adult Health Study sample in Hiroshima and Nagasaki were examined for positive rate of HTLV-I antibody. Several lymphocyte parameters were also studied for 70 antibody-positive subjects in Nagasaki. The HTLV-I antibody-positive rate was higher in Nagasaki (6.36\%) than in Hiroshima ( $0.79 \%$ ) and significantly increased with increasing age, but no association was observed with radiation dose. Whether relationship existed between antibody titer levels and radiation dose among antibody-positive subjects was not clear. The frequency of abnormal lymphocytes tended to be higher in antibody-positive subjects than in antibody-negative subjects, and higher in females than in males regardless of radiation dose. The lymphocyte count was lower in antibody-positive subjects than in antibody-negative subjects and lower in female than in male subjects. No evidence was found to suggest that atomic-bomb radiation plays an important role in HTLV-I infection. 
RERF Report No. 16-94 Height reduction among prenatally exposed atomic-bomb survivors: A longitudinal study of growth. E Nakashima, RL Carter, K Neriishi, S Tanaka, S Funamoto. (Published in Health Phys 68:766-72, 1995.)

Using a random coefficient regression model, sex-specific longitudinal analyses of height were made on 801 (392 male and 409 female) atomic-bomb survivors exposed in utero to detect dose effects on standing height. The data set resulted from repeated measurements of standing height of adolescents (age 10-18 y). The dose effect, if any, was assumed to be linear. Gestational ages at the time of radiation exposure were divided into trimesters. Since an earlier longitudinal data analysis has demonstrated radiation effects on height, the emphasis in this paper is on the interaction between dose and gestational age at exposure and radiation effects on the age of occurrence of the adolescent growth spurt. For males, a cubic polynomial growth-curve model applied to the data was affected significantly by radiation. The dose by trimester interaction effect was not significant. The onset of adolescent growth spurt was estimated at about 13 y at $0 \mathrm{~Gy}$. There was no effect of radiation on the adolescent growth spurt. For females, a quadratic polynomial growth-curve model was fitted to the data. The dose effect was significant, while the dose by trimester interaction was again not significant.

RERF Report No. 17-94 Further observations on abnormal brain development caused by prenatal A-bomb exposure to ionizing radiation. $H$ Yoshimaru, $M$ Otake, WJ Schull, S Funamoto. (Published in Int J Radiat Biol 67:359-71, 1995.)

The pervasiveness of abnormal brain development caused by prenatal exposure to ionizing radiation is still largely unknown. The relationship between A-bomb radiation dose and two measures of neuromuscular performance, one of grip strength and the other of the fine motor coordination required in repetitive action, is described. A multivariate analysis of covariance was used to evaluate the effect of several covariates, such as prenatal radiation exposure and some physical measurements or IQ, adding city and sex as categorical factors. When mentally retarded cases were included, a statistically significant effect of radiation exposure on the grip strength and repetitiveaction test scores was seen in the 8-15-week postovulation period, and a statistically suggestive effect at 16-25-week postovulation. No effect of radiation exposure on the two test scores was noted for prenatal exposure in either of the aforementioned periods when mentally retarded cases were excluded, but a statistically significant diminution of IQ was noted for exposures $\geq 16$-week postovulation. We discuss, from the biological perspective, the projected standard scores for exposures $\geq 16$-week postovulation, and the possibility of lower IQ, small head and mild mental retardation related to radiation exposures $\leq 15$-week postovulation with mentally retarded cases excluded. 
RERF Report No. 18-94 Relationship between cataracts and epilation in atomic bomb survivors. K Neriishi, FL Wong, E Nakashima, M Otake, K Kodama, K Choshi. (Published in Radiat Res 144:107-13, 1995.)

Among 1713 atomic bomb survivors who underwent ophthalmological examinations from 1963-1964, the risk of cataract formation per unit dose of radiation was significantly greater for those who reported hair loss of $67 \%$ or more after exposure (the epilation group) than for those who reported less or no hair loss (the no-epilation group) $(P<0.01)$. Such an epilation effect has also been associated with leukemia mortality and the frequency of chromosome aberrations. Although this might be interpreted as indicating differential sensitivity to radiation between the epilation group and the no-epilation group, it could also be explained by imprecision in dose estimates. We have calculated that a $48 \%$ random error in DS86 dose estimates could be in accordance with the dose-response relationship for the prevalence of cataracts in the epilation group or the no-epilation group. Possible mechanisms for variation in radiosensitivity are discussed.

RERF Report No. 21-94 High yield of restriction fragment length polymorphisms in two-dimensional separations of human genomic DNA. R Kuick, J Asakawa, JV Neel, C Satoh, SM Hanash. (Published in Genomics 25:345-53, 1995.)

We have investigated the extent to which restriction fragment length polymorphism can be detected by two-dimensional electrophoresis of end-labeled genomic restriction fragments. Genomic DNA was digested with NotI and EcoRV and labeled at the NotI recognition site before first-dimension electrophoresis in disk gels. DNA in the disk gels was further digested in situ with Hinfl prior to second-dimension electrophoresis, yielding patterns in which approximately 2000 end-labeled fragments were simultaneously visualized. On the basis of studies of 6 mother/father/child trios, a group of 184 fragments was organized into 85 polymorphic systems in which all allelic fragments were detectable in the 2-D patterns. Another 206 fragments varied as to their presence among individuals, but their relatedness to other fragments was not established. Our data indicate that a large number of DNA polymorphisms can be simultaneously scored in 2-D separations of genomic DNA fragments.

RERF Report No. 22-94 Incidence of benign gastrointestinal tumors among atomic bomb survivors. E Ron, FL Wong, $\mathrm{K}$ Mabuchi. (Published in Am J Epidemiol $142: 68-75,1995).(J)$

Using the Hiroshima and Nagasaki tumor and tissue registries, benign tumors of the stomach, colon, and rectum were identified among members of the Life Span Study cohort of atomic bomb survivors. During the period 1958-1989, a total of 470 cases with histologically confirmed benign gastrointestinal tumors (163 stomach, 215 colon, 
and 92 rectum) were identified among approximately 80,000 Life Span Study members with known radiation doses, who were alive in 1958. Restricting the analysis to adenomatous tumors not detected at autopsy, a dose-response relation was observed for stomach tumors (excess relative risk at 1 sievert $\left[E R R_{1 S v}\right]=0.53 ; 95 \%$ confidence interval $[\mathrm{CI}]-0.01$ to 1.43 ). However, there was little evidence of a dose response for colon tumors $\left(E R R_{I S v}=0.14 ; 95 \% \mathrm{CI}-0.20\right.$ to 0.76$)$, and no evidence was present for rectal tumors $\left(E R R_{I S v}=-0.25 ; 95 \% \mathrm{CI}\right.$ undetermined to 0.80$)$. The excess relative risk (ERR) for benign tumors of the stomach is consistent with the excess found for stomach cancer. For cancer of the rectum, the dose response was not significant, but the point estimate of the excess relative risk was positive. The excess relative risk for benign colon tumors is less than that reported for colon cancer $\left(E R R_{I S v}=0.72\right)$. The authors observed a dramatic increase in colon tumors detected after 1985, suggesting that the relatively recent introduction of colonoscopy may be influencing these results.

RERF Report No. 24-94 Lack of effects of atomic bomb radiation on genetic instability of tandem-repetitive elements in human germ cells. M Kodaira, C Satoh, K Hiyama, K Toyama. (Published in Am J Hum Genet 57:1275-83, 1995.)

In a pilot study to detect the potential effects of atomic bomb radiation on germ-line instability, we screened 64 children from 50 exposed families and 60 from 50 control families for mutations at six minisatellite loci by using Southern blot analysis with Pc-1, $\lambda$ TM-18, ChdTC-15, p $\lambda \mathrm{g} 3, \lambda \mathrm{MS}-1$, and CEB-1 probes. In the exposed families, one or both parents received a radiation dose $>0.01 \mathrm{~Sv}$. Among the 64 children, only one child had parents who were both exposed. Thus, of a total of 128 gametes that produced the 64 children, 65 gametes were derived from exposed parents and 63 were from unexposed parents, the latter being included in a group of 183 unexposed gametes used for calculating mutation rates. The average parental gonadal dose for the 65 gametes was $1.9 \mathrm{~Sv}$. We detected a total of 28 mutations at the $p \lambda \mathrm{g} 3, \lambda M S-1$, and $C E B-1$ loci, but no mutations at the PC- $1, \lambda T M-18$, and $C h d T C-15$ loci. We detected 6 mutations in 390 alleles of the 65 exposed gametes and 22 mutations in 1098 alleles of the 183 gametes from the unexposed parents. The mean mutation rate per locus per gamete in these six minisatellite loci was $1.5 \%$ in the exposed parents and $2.0 \%$ in the unexposed parents. We observed no significant difference in mutation rates in the children of the exposed and the unexposed parents $(P=0.37$, Fisher's exact probability test). 
RERF Report No. 25-94 Prevalence of hepatitis B surface antigen, hepatitis $B$ e antigen and antibody, and antigen subtypes in atomic bomb survivors. $K$ Neriishi, S Akiba, T Amano, $\mathrm{T}$ Ogino, $\mathrm{K}$ Kodama. (Published in Radiat Res 144:215-21, 1995.)

On the basis of previous studies showing an association between hepatitis $B$ surface antigen (HBsAg) positivity and radiation exposure in atomic bomb (A-bomb) survivors, we investigated further the active state of hepatitis $B$ virus (HBV) infection by incorporating tests for hepatitis $\mathrm{B}$ e antigen ( $\mathrm{HBeAg}$ ) and hepatitis $\mathrm{B}$ e antibody (anti$\mathrm{HBe}$ ) and HBsAg subtypes into our biennial health examinations. Among $6548 \mathrm{~A}-$ bomb survivors for whom HBsAg was assayed between July 1979 and July 1981, 129 persons were HBsAg positive. $\mathrm{HBeAg}$ and anti-HBe were measured in 104 of these persons and subtypes of $\mathrm{HBsAg}$ in 98 persons. Among those exposed to radiation (average liver dose $0.58 \mathrm{~Sv}$ ), the odds ratio of $\mathrm{HBsAg}$ positivity tended to increase with radiation dose ( $P$ for trend $=0.024$ ). The $P$ values for association between the prevalence of HB e antigen and radiation dose and between the prevalence of anti-HBe and radiation dose were 0.094 and 0.17 , respectively. The $\mathrm{HB}$ antigen subtype adr was predominant over other subtypes in both Hiroshima and Nagasaki, but the distribution of subtypes did not seem to differ in relation to radiation dose. These results suggested that A-bomb survivors remain in an active state of HBV infection and that the mechanism(s) of seroconversion may be impaired.

RERF Report No. 2-95 Mutation frequency in human blood cells increases with age. M Akiyama, S Kyoizumi, Y Hirai, Y Kusunoki, KS Iwamoto, N Nakamura. (Published in Mutat Res 338:141-9, 1995.)

Using either the colony formation assay or flow cytometry, it is feasible to measure the frequency of rare mutant lymphocytes or erythrocytes in human peripheral blood. Accordingly, we have investigated the mutant cell frequencies of the hypoxanthineguanine phosphoribosyltransferase and T-cell receptor genes in T lymphocytes and of the glycophorin A gene in erythrocytes of several hundred persons aged 0-96 years. The mutant frequency of every one of these genes increased significantly with age. A simple accumulation of mutations in hematopoietic stem cells over time may explain the age-dependent increase in the frequency of glycophorin A mutants. In contrast, a balance between mutant cell generation and loss should be taken into account for the mechanism of the increase of T-cell mutations. 
RERF Report No. 5-95 Late effects of radiation on the human immune system: An overview of immune response among the atomic-bomb survivors. $M$ Akiyama. (Published in Int J Radiat Biol 68:497-508, 1995.)

The studies of the late effects of atomic-bomb (A-bomb) radiation on the immune system were started about 20 years after the bombings in 1945 . The most remarkable late effects of radiation are the functional and quantitative abnormalities of $T$ and $B$ cells in survivors exposed to high doses ( $\geq 1.0 \mathrm{~Gy})$. Abnormalities of T-cell immunity include: (1) a decreased proportion of $\mathrm{CD}^{+} \mathrm{T}$ cells in peripheral blood lymphocytes, particularly the proportion of $\mathrm{CD}^{+} \mathrm{CD}^{+} \mathrm{RA}^{+}$naive $\mathrm{T}$ cells (study period 1987-1991); (2) an increased frequency of $\mathrm{CD}^{-}$and $\mathrm{CD} 8^{-}$(double negative) $\alpha \beta^{+} \mathrm{T}$ cells (19871991); and (3) functional defects in T-cell responses to mitogens and alloantigens (1974-1985). B-cell abnormalities include: (1) a significant increase in the proportion of B cells among peripheral lymphocytes (1987-1991); (2) an increase in serum immunoglobulin $A$ levels in females and immunoglobulin $\mathrm{M}$ and the incidence of rheumatoid factor in both sexes (1987-1989); and (3) an increased level of anti-EpsteinBarr virus antibody titer (1987-1990). In contrast, suggestive $(0.05<p<0.1)$ or not significant $(p>0.1)$ dose effects were observed for the number and function of natural killer cells (1983-1991), and benign monoclonal gammopathy (1979-1987). In addition, studies initiated sooner after the bombing such as the incidence of autoimmune diseases (1958-1987), systemic bacterial infections (1954-1967), and granulocyte functions (1947-1979) also show little dose-effects. Thus, A-bomb radiation induced the alteration of the balance/interaction between the T- and B-cell subsetsspecifically, a decrease in the T-cell population and an increase in the B-cell population in the periphery.

RERF Report No. 9-95 Spectrum of in vivo hprt mutations in T lymphocytes from atomic bomb survivors. I. Sequence alterations in cDNA. $H$ Shimahara, $T$ Kato, Y Hirai, M Akiyama. (Published in Carcinogenesis 16:583-91, 1995.)

Recently, we found an elevated frequency of 6-thioguanine-resistant ( $\mathrm{TG}^{\mathrm{T}}$ ) mutations at the hypoxanthine-guanine phosphoribosyltransferase ( $h p r t)$ gene in T cells of peripheral blood from atomic bomb survivors and a slight, but significant, positive correlation between the frequency of mutation and radiation dose. Southern blot analysis of DNA from $\mathrm{TG}^{\mathrm{r}}$ mutant $\mathrm{T}$ cells of atomic bomb survivors, however, failed to show a significant difference between the control and survivor groups. We here report mutational events at the $h p r t$ locus of $\mathrm{TG}^{r}$ mutant $\mathrm{T}$ cell clones from atomic bomb survivors as found by (i) the multiplex polymerase chain reaction (PCR) and (ii) the reverse transcription (RT)-PCR of cDNA and sequencing. The numbers of independent TG $^{r}$ mutant $T$ cell clones examined were 41 from a control group of 18 individuals who had received less than $0.005 \mathrm{~Gy}$ and 50 from an exposed group of 24 individuals who had received more than $1.5 \mathrm{~Gy}$ (mean dose $2.45 \pm 0.85 \mathrm{~Gy}$ ). Gross structural alterations, 
which were detected by multiplex PCR as a loss of or shift in hprt exon-containing fragments of genomic DNA, were found in $10-15 \%$ of the clones from both groups, thus indicating that there was no significant difference between them. The altered sequences in the HPRT cDNAs recovered from both groups were of various types. Similar proportions of base substitutions $(\sim 45 \%)$, deletions or insertions $(\sim 25 \%)$ and exon skipping $(-20 \%)$ were found in both groups, indicating that neither the gross structural alterations in the genomic DNA nor sequence alterations in the hprt cDNA of both groups differ significantly. These results confirm our previous observation that A-bomb-induced HPRT mutant $T$ cells have mostly been eliminated from the peripheral blood over the decades that have elapsed since exposure. Some unique features of the mutational sequence alterations found are also described.

RERF Report No. 10-95 Activation of telomerase in human lymphocytes and hematopoietic progenitor cells. $\mathrm{K}$ Hiyama, Y Hirai, S Kyoizumi, M Akiyama, E Hiyama, MA Piatyszek, JW Shay, S Ishioka, M Yamakido. (Published in J Immunol 155:3711-5, 1995.)

This is the first report describing up-regulation of telomerase activity in human normal cells. Telomerase, a ribonucleoprotein enzyme, has been thought to be involved in maintaining telomere length stability in germ-line and most cancer cells, but not in normal cells. However, in the present study, we demonstrate that telomerase activity is detectable at low levels in normal human $\mathrm{T}$ and $\mathrm{B}$ cells, increases by in vitro mitogenic stimulation, increases in hematopoietic progenitor cells upon their proliferation and differentiation, and decreases with aging. Understanding the regulation of telomerase activity in normal cells may provide important insights not only into the mechanisms of normal cellular senescence but also into the mechanisms of telomerase activity deregulation as part of cancer development.

\section{Commentary and Review Series}

Editor's note: Reports in the Commentary and Review (CR) Series begin as manuscripts that are officially approved by the RERF permanent directors for submission to journals. Upon their acceptance by a journal, such manuscripts are designated as $C R$ reports. Reports in the CR series consist of journal-article reprints that are purchased from the publishers and bound in CR covers with a Japanese summary. $C R$ reports are published to rapidly disseminate ideas, discussions, comments, and recommendations on research carried out by RERF scientists and directors. This series also includes working papers or invited presentations prepared for national and international organizations or conferences, discussions of research concerning atomic-bomb survivors carried out elsewhere, and in general, materials of lasting importance to RERF and atomic-bomb survivor research. 
CR Report No. 3-93 Analysis of chromosome aberration data with measurement error from atomic-bomb survivors: Utilization of large-scale external information. E Nakashima, $\mathrm{K}$ Ohtaki. (Published in Jpn J Biometrics 16:19-36, 1995.)

Overdispersion data of chromosome-aberration rates from atomic-bomb survivors were analyzed by the quasilikelihood/pseudolikelihood (QL/PL) estimating equation method (Breslow 1990). The variance function was composed of two extra-binomial variations: one is an intra-individual correlation, the other is a variation from dosimetry error. This dose-error-variance component was derived using the quasi-structural method (Pierce, Stram, Vaeth and Schafer 1992) that incorporates large-scale external dosimetry information. Using the results of fitting the QL/PL estimating equation, the Wald test revealed that the dose-estimation error is a significant source of overdispersion.

CR Report No. 1-96 A simple reductionist model for cancer risk in atom bomb survivors. ML Mendelsohn. (Published in Modeling of Biological Effects and Risks of Radiation Exposure [NIRS Symposium Series No. 26], edited by J Inaba, S Kobayashi. Chiba, Japan, National Institute of Radiological Sciences, 1995. pp 18592)

1) In data from the atom bomb survivors of Hiroshima and Nagasaki, the roughly linear-quadratic radiation dose responses for chromosome aberration and leukemia correspond closely to each other, as do the linear dose responses for gene mutation and solid cancer incidence.

2) In view of the increasing evidence for multiple oncogene and suppressor gene changes in human cancer, as well as the evidence that human cancer rate is often proportional to age to the power of 6 or so, it is postulated that the radiation has contributed one and only one oncogenic mutational event to the radiation induced cancers.

3) The radiation-induced cancers should therefore display a cancer rate versus age relationship that has a power of $n-1$, where $n$ is the power for the corresponding background cancers.

4) It is shown that this is precisely what is happening in the collective solid cancer incidence of the atom bomb survivors. 


\section{Publications in the Scientific Literature}

\section{April 1995-31 March 1996}

Editor's note: These journal articles differ from RERF Reports (see preceding section) in the following ways. Most are based on collaborative studies with outside researchers and/or the work was unrelated to a specific RERF research protocol. Some articles may be invited summaries of reanalyses of previously reported RERF findings.

\section{Peer-reviewed journal articles}

Asakawa J, Kuick R, Neel JV, Kodaira M, Satoh C, Hanash SM: Quantitative and qualitative genetic variation in two-dimensional DNA gels of human lymphocytoid cell lines. Electrophoresis 16:241-52, 1995

Nakashima E: Analysis of count data using power variance function. J Jpn Stat Soc 25:193-204, 1995

Ron E, Preston DL, Mabuchi K: More about cancer incidence in atomic bomb survivors: solid tumors, 1958-1987 (Letter to the editor). Radiat Res 141:126-7, 1995

Shigematsu I, Mendelsohn ML: The Radiation Effects Research Foundation of Hiroshima and Nagasaki. Past, present, and future. JAMA 274:425-6, 1995

Teraoka S, Kyoizumi S, Seyama T, Yamakido M, Akiyama M: A novel SCID mouse model for studying spontaneous metastasis of human lung cancer to human tissue. Jpn $J$ Cancer Res 86:419-23, 1995

Teraoka S, Kyoizumi S, Suzuki T, Yamakido M, Akiyama M: Growth suppressive efficacy of human LAK cells against human lung cancer implanted into SCID mice. Int J Oncol 6:1271-7, 1995

Yamamoto O, Seyama T, Jo T, Terato H, Saito T, Kinomura A: Oral administration of tritiated water (HTO) in mouse. II. Tumor development. Int J Radiat Biol 68:47-54, 1995

Yanagihara $K$, Nii $M$, Tsumuraya $M$, Numoto $M$, Seito T, Seyama T: A radiationinduced murine ovarian granulosa cell tumor line: introduction of v-ras gene potentiates a high metastatic ability. Jpn J Cancer Res 86:347-56, 1995 
Yonehara H, Aoyama T, Radford EP, Kato H, Sakanoue M: Radon concentrations in residential housing in Hiroshima and Nagasaki. Health Phys 68:683-8, 1995

\section{Articles in proceedings}

From Assessment of Radiation Effects by Molecular and Cellular Approaches. Ed by Fliedner TM, Cronkite EP, Bond VP. Dayton, Ohio, AlphaMed Press, 1995. (Stem Cells Volume 13, Supplement 1, 1995)

Akleyev AV, Kossenko MM, Silkina LA, Degteva MO, Yachmenyov VA, Awa AA, Akiyama M, Veremeyeva GA, Vozilova AV, Kyoizumi S, Kozheurov VP, Vyushkova OV: Health effects of radiation incidents in the Southern Urals. pp 58-68

Preston DL: Cancer risks and biomarker studies in the atomic bomb survivors. pp 40-8

From Radiobiological Concepts in Radiotherapy. Ed by Bhattacharjee D, Singh BB. New Delhi, India, Narosa Publishing House, 1995. (Proceedings of International Symposium on Emerging Frontiers in Radiation Biology, Bombay, 1-3 December 1993)

Ban S, Sugahara T: Radiosensitivity of skin fibroblasts and lymphocytes from atomic bomb survivors in Hiroshima. pp 82-7

From Modeling of Biological Effects and Risks of Radiation Exposure (NIRS Symposium Series No. 26). Ed by Inaba J, Kobayashi S. Chiba, Japan, National Institute of Radiological Sciences, 1995. (Proceedings of the 26th National Institute of Radiological Sciences Symposium, Chiba, Japan, 8-9 December 1994) Mabuchi K: Issues in risk modeling. pp 215-9 (In Japanese)

From the Proceedings of the 36th Late A-bomb Effects Research Meeting, 1995. All articles are in Japanese.

Asakawa J, Kodaira M, Kaneoka S, Satoh C: A new method for the mutation study on the children of the atomic bomb survivors: two-dimensional electrophoresis of DNA. Hiroshima Igaku [J Hiroshima Med Assoc] 49:431-3, 1996

Ashizawa K, Yamashita S, Namba H, Yokoyama N, Ito M, Hoshi M, Shibata Y, Nagataki S: Childhood thyroid diseases and whole body Cs-137 concentration around Chernobyl. Hiroshima Igaku [J Hiroshima Med Assoc] 49:388-90, 1996

Ishioka N, Kyoizumi S, Umeki S, Hirai Y, Suzuki T, Kodama T, Ohama K, Akiyama M: Early detection of in vivo T-cell receptor mutations induced by radiation exposure. Hiroshima Igaku [J Hiroshima Med Assoc] 49:437-40, 1996 
Ito M, Yamashita S, Ashizawa K, Hara T, Namba H, Hoshi M, Shibata Y, Sekine I, Panasyuk GD, Nagataki S: Histopathological characteristics of pediatric thyroid cancer around Chernobyl. Hiroshima Igaku [J Hiroshima Med Assoc] 49:361-3, 1996

Kodama Y, Pawel DJ, Nakano M, Ohtaki K, Nakamura N, Awa AA: Frequencies of stable chromosome aberrations in A-bomb survivors-variation in dose-response relationship by shielding categories. Hiroshima Igaku [J Hiroshima Med Assoc] 49:319-21, 1996

Mabuchi K, Pierce DA, Shimizu Y, Preston DL: Fifty years after the A-bombs and the current cancer risk. Hiroshima Igaku [J Hiroshima Med Assoc] 49:277-9, 1996

Matsuura M, Hayakawa N, Hoshi M, Shimokata H, Ohtaki M, Kasagi F, Ikeuchi M, Sumida H, Hiraoka M: Risk analyses of mortality due to stomach, lung, breast and colon cancer among atomic-bomb survivors in Hiroshima Prefecture based on ABS93D. Hiroshima Igaku [J Hiroshima Med Assoc] 49:310-3, 1996

Nakamura N, Miyazawa C, Sawada S, Akiyama M, Awa AA: Radiation-dose estimation by ESR using teeth donated by A-bomb survivors. Hiroshima Igaku [ $J$ Hiroshima Med Assoc] 49:314-8, 1996

Nakano M, Kodama Y, Ohtaki K, Nakamura N, Awa AA: Detection of stable chromosome aberrations in A-bomb survivors - comparison of conventional staining and FISH methods. Hiroshima Igaku [J Hiroshima Med Assoc] 49:322-3, 1996

Nakashima E, Nakamura Y, Preston DL: Analysis of total solid cancer incidence among atomic-bomb survivors using Moolgavkar-Venzon-Knudson carcinogenesis model. Hiroshima Igaku [J Hiroshima Med Assoc] 49:306-9, 1996

Satoh C, Yasunaga K, Miura A: Effects of atomic bomb radiation on genetic instability of microsatellites in human germ cells. Hiroshima Igaku [J Hiroshima Med Assoc] $49: 423-6,1996$

Suzuki T, Kyoizumi S, Umeki S, Akiyama M, Aizawa S: T-cell receptor gene mutations in p53 gene deficient mice. Hiroshima Igaku [J Hiroshima Med Assoc] 49:468-70, 1996

Takahashi K, Hirai Y, Kitahira M, Ishioka N, Kusunoki Y, Kyoizumi S, Akiyama M: Development of a novel assay system to detect somatic mutations using neutrophils and a study of atomic-bomb survivors. Hiroshima Igaku [J Hiroshima Med Assoc] 49:434-6, 1996 
Takahashi N, Kaneko J, Satoh C: Quantitative analysis of bands on a Southern filter using nonradioisotope (digoxigenin)-labeled probes. Hiroshima Igaku [J Hiroshima Med Assoc] 49:448-50, 1996

Tsuyama N, Seyama T, Iwamoto KS, Mizuno T, Akiyama M: Mechanism of sdil/WAF1 gene induction by ionizing radiation. Hiroshima Igaku [J Hiroshima Med Assoc] 49:451-4, 1996

Yamada M: Psychical and psychological effects of A-bomb radiation. Hiroshima Igaku [J Hiroshima Med Assoc] 49:294-6, 1996

\section{Invited articles or special reports in Japanese journals}

Kyoizumi S: Experimental medicine using the SCID-hu mouse model. Anitechs 7:247-51, 1995

Kyoizumi S: Somatic mutations and radiation carcinogenesis-Implications of erythrocytic GPA mutations in atomic bomb survivors. Hoshasen Seibutsu Kenkyu [Radiat Biol Res Commun] 30:201-24, 1995

Kyoizumi S, Umeki S, Akiyama M: Assay system of human somatic mutations using T-cell antigen receptor gene as a marker and its application to biodosimetry. Kankyo Henigen Kenkyu [Environ Mutagen Res Commun] 17:151-60, 1995

\section{Contributions to RERF Section of the Journal of the Hiroshima Medical Association.}

RERF: Radiation Effects Research Foundation bibliography of publications, 1994. Hiroshima Igaku [J Hiroshima Med Assoc] 48:1103-6, 1995

RERF: The 22nd RERF Scientific Councilors Meeting (Brief report). Hiroshima Igaku [J Hiroshima Med Assoc] 48:831, 1995

RERF: RERF directors, supervisors, and scientific councilors (as of September 1995). Hiroshima Igaku [J Hiroshima Med Assoc] 48:1213, 1995

\section{Contributions to RERF Section of the Journal of the Hiroshima Medical Association. (Japanese translation of the RERF Update articles)}

Izumi S, Pierce DA, Preston DL, Delongchamp RR, Yoshimoto Y, Mabuchi K: Facts and figures. Part 1. Dosimetry System 1986 Dose: distance and shielding. Part 2. Inutero-exposed atomic-bomb survivors: cancer risk update. Hiroshima Igaku [J Hiroshima Med Assoc] 48:587-8, 1995 
Kusunoki Y, Kodama Y, Hirai Y, Nakamura N, Akiyama M: The origin of clonal chromosome aberrations. Hiroshima Igaku [J Hiroshima Med Assoc] 48:480-3, 1995

Ueda $\mathrm{H}$ : Arteriosclerosis in the atomic-bomb survivors. Pulse-wave velocity measurements suggest a relationship between radiation exposure and the prevalence of arteriosclerosis. Hiroshima Igaku [J Hiroshima Med Assoc] 49:107-8, 1996

\section{Reports of research groups related to the Japanese Ministry of Health and Welfare}

From the FY1994 Report of A-bomb Disease Research Teams. Tokyo, Nippon Koshueisei Kyokai-Jpn Public Health Assoc, 1995. All articles are in Japanese.

Akahoshi M: Mild depression among atomic-bomb survivors. pp 31-2

Fujita Y, Mabuchi K, Ito C: Mortality follow-up survey using National Death Index (NDI). pp 13-4

Kasagi F, Kodama K, Yamada M: Study to grasp the actual state of aging in a population of atomic-bomb survivors: significance of record linkage of medical information. pp 76-8

Kusumi S, Tsuruta M, Akahoshi M, Kodama K, Moriya T, Sasaki F, Tanaka J, Yoshizawa H: Seroepidemiological study of liver diseases among atomic-bomb survivors. pp 17-20

Mori T, Akiyama M: Gene analysis of malignant tumors in Thorotrast-disease patients. pp 40-2

Neriishi K, Yoshimoto Y, Kodama K: Longitudinal study of M-proteinemia among atomic-bomb survivors (preliminary analysis). pp 33-5 


\section{Oral Presentations}

\section{April 1995-31 March 1996}

Editor's note: The asterisk indicates the person who presented the talk.

The 43rd Annual Scientific Meeting of the Radiation Research Society, 1-6 April 1995, San Jose, California

n Cancer mortality in the A-bomb survivors, 1950-1990

Preston DL,* Pierce DA, Mabuchi K, Shimizu Y

The 59th Scientific Meeting of the Japanese Circulation Society, 1-3 April 1995, Nagoya

- Effects of age at menopause on risks for ischemic heart disease-A follow-up study in the 12-year perimenopausal period

Akahoshi M,* Soda M, Tsuruta M, Ichimaru S, Seto S, Yano K

- Study of actual state of deaths occurring within 24 hours of onset of disease

Ueda H,* Kodama K, Kasagi F, Fujita Y

The 24th General Assembly of the Japan Medical Congress, 7-9 April 1995, Nagoya

- Research on the nuclear accident in the South Uralian region

Mabuchi K*

- Epidemiology of radiation effects on health

Kodama $\mathrm{K}^{*}$

The 84th Annual Meeting of the Japanese Society of Pathology, 17-19 April 1995, Nagoya

- Study of the incidence of tumors of the central nervous system among A-bomb survivors

Yonehara S, * Fujii H, Kishikawa M, Kobuke T, Tokunaga M, Tokuoka S, Preston DL, Mabuchi K

- Study of the incidence of salivary gland tumors among A-bomb survivors

Saku T,* Hayashi Y, Takahara O, Matsuura H, Tokunaga M, Tokuoka S, Preston DL, Mabuchi K

- Study of the skin cancer incidence among A-bomb survivors

Kishikawa M,* Kobuke T, Iseki M, Tokunaga M, Tokuoka S, Preston DL, Mabuchi K 
The 9th Annual Meeting of Japanese Society of Computational Statistics, 26-28 April 1995, Okinawa

- Risk and threshold estimation of mental retardation Otake $\mathrm{M}^{*}$

The 6th Annual Space Radiation Health Investigators' Workshop, 2-5 May 1995, New York

- Cancer risks in atomic bomb survivors Preston DL*

The 16th Annual Scientific Session of North American Society of Pacing and Electrophysiology, 3-6 May 1995, Boston, Massachusetts

- Long-term follow-up study of Wolff-Parkinson-White (WPW) syndrome in Hiroshima and Nagasaki, Japan

Hayano M,* Ueyama C, Konoe A, Hirata T, Hano O, Yano K, Akahoshi M, Kodama K

International Conference "Actual and Prognosed Mental Health Disorders after Nuclear Catastrophe in Chernobyl”, 23-30 May 1995, Kiev, Ukraine

- Study of long-term psychological disorders among the atomic-bomb survivors Neriishi K, * Yamada M, Kodama K, Delongchamp RR, Shigematsu I

The 36th Late A-bomb Effects Research Meeting, 28 May 1995, Hiroshima

- Relationship between HBs antigen and epilation in atomic bomb survivors Neriishi K, * Akiba S, Amano T, Ogino T, Kodama K

- Analysis of total solid cancer incidence among atomic-bomb survivors using Moorgavkar-Venzon-Knudson carcinogenesis model Nakashima E, * Nakamura Y, Preston DL

- Quantitative analysis of bands on a southern filter using nonradioisotope (digoxigenin)-labelled probes Takahashi N,* Kaneko J, Satoh C

- Effects of atomic bomb radiation on genetic instability of microsatellites in human germ cells

Satoh $\mathrm{C}$,* Yasunaga K, Miura A

- A new method for the mutation study on the children of the atomic bomb survivors: Two-dimensional electrophoresis of DNA Asakawa J, * Kodaira M, Kaneoka S, Satoh C

- Radiation dose estimation by ESR for teeth donated by A-bomb survivors Nakamura N,* Iwasaki M, Miyazawa C, Niwa K, Sawada S, Akiyama M, Awa AA 
- Frequencies of stable chromosome aberrations in A-bomb survivors-Variation in dose-response relationship by shielding categories

Kodama Y,* Pawel DJ, Nakano M, Ohtaki K, Nakamura N, Awa AA

- Detection of stable chromosome aberrations in A-bomb survivors-Comparison of conventional staining and FISH methods

Nakano M, Kodama Y, Ohtaki K, Nakamura N, Awa AA

- Analysis of induction mechanism of sdil/WAF1 gene by ionizing radiation Tsuyama N,* Seyama T, Iwamoto KS, Mizuno T, Akiyama M

- Early detection of mutations of T-cell antigen receptor (TCR) genes Ishioka N,* Kyoizumi S, Umeki S, Hirai Y, Suzuki T, Kodama T, Ohama K, Akiyama $\mathrm{M}$

- Mutation of T-cell receptor genes in p53-deficient mice Suzuki T,* Kyoizumi S, Umeki S, Aizawa S, Akiyama M

- Development of a novel assay system to detect somatic mutations using neutrophils and a study of A-bomb survivors

Takahashi K, ${ }^{*}$ Hirai Y, Kitahira M, Ishioka N, Kusunoki Y, Kyoizumi S, Akiyama $\mathrm{M}$

- Association between thyroid diseases and HTLV-I infection in Nagasaki Tsuruta M,* Yokoyama N, Shibata Y, Akahoshi M, Matsuo T, Tomonaga M, Nagataki $S$

Application of Health Risk Assessment-Today and Tomorrow, 29 May 1995, Hiroshima

- Analysis of human radiation risk using SCID-hu mouse model Kyoizumi $\mathrm{S*}^{*}$

- Detection of somatic mutation and its application to risk evaluation Akiyama $\mathrm{M}$, Kyoizumi S, Cologne JB

The 12th NIRS-RERF-RIRBM Research Exchange Seminar, 29 June-1 July 1995, Chiba

- Spontaneous and X-radiation induced genomic instability in a p53-mutant-type dependent manner at the TCR gene

Iwamoto $\mathrm{KS}^{*}$

- Analysis of acute radiation damages in humans using SCID-hu mouse model Kyoizumi $S^{*}$

- Abnormalities of genes and functions of blood stem cells of A-bomb survivors Hirai $Y^{*}$ 
The 26th Meeting of Japan Nursing Society, 6-7 July 1995, Kohriyama

- Understanding and present state of medicine-taking of middle and advanced age people

Matsuoka K,* Yamashita M

The 3rd Workshop of Health Research Foundation, 18-20 July 1995, Nagasaki

a Mutation frequency in human blood cells increases with age Akiyama M,* Kyoizumi S, Hirai Y, Kusunoki Y, Iwamoto KS, Nakamura N

The 9th International Congress of Immunology, 23-29 July 1995, San Francisco, California

- Cloning of full length cDNA capable of complementing SCID mouse mutation Hamatani $\mathrm{K}$, Araki $\mathrm{R}$, Itoh $\mathrm{M}$, Abe $\mathrm{M}$

- Analysis of $\mathrm{V}(\mathrm{D}) \mathrm{J}$ recombination products of SCID lymphoid organs Abe $M, *$ Hamatani $K$, Itoh $M$, Araki $R$

The 63rd Annual Meeting of the Japan Statistical Society, 24-27 July 1995, Ohita - Comparison of some methods for estimation in Poisson models with overdispersion

Nakashima $E^{*}$

International Symposium of 50th Anniversary of A-bombings, 31 July 1995, Hiroshima

- Genetic effects of atomic radiation, Hiroshima and Nagasaki Awa $\mathrm{AA}^{*}$

The 13th General Meeting of Japanese Society of Bone Metabolism, 17-19 August 1995, Fukuoka

- A study of vertebral fracture and its risk factors in the last 10 years

Fujiwara S,* Kodama K, Kasagi F

The 2nd International Conference on Environmental Mutagens in Human Populations, 20-25 August 1995, Prague, Czech Republic

- Detection of somatic mutation and its application to risk evaluation Akiyama M,* Kyoizumi S, Cologne JB

- Genetic analysis of children of atomic bomb survivors Satoh C,* Takahashi N, Asakawa J, Kodaira M 
The 10th International Congress of Radiation Research, 27 August-1 September 1995, Würzburg, Germany

n Germline mutations in the children of the A-bomb survivors Kodaira $M, *$ Satoh $C$

- Study on the effects of atomic bombs on germ cell mutations Satoh C,* Takahashi N, Asakawa J, Kodaira M

- Deletion of chromosome 5 in lymphocytes from atomic-bomb survivors Ohtaki K,* Nakamura N, Awa AA

n Comparison of stable chromosome aberration frequencies determined using FISH and conventional methods in A-bomb survivors Nakano M,* Kodama Y, Ohtaki K, Awa AA, Nakamura N

- Atomic-bomb-radiation cataract and the occurrence of severe epilation based on a threshold model Otake M,* Neriishi K, Schull WJ

- Fifty years after the A-bomb and the current cancer risk Mabuchi K, * Preston DL, Pierce DA, Shimizu Y, Vaeth M

n The offspring $\left(\mathrm{F}_{1}\right)$ of atomic-bomb survivors: Mortality and incidence data update Yoshimoto Y,* Soda M, Mabuchi K

- Incidence of myocardial infarction in atomic-bomb survivors Kodama K, * Shimizu Y, Kasagi F, Ueda H, Yamada M

- Spontaneous and X-radiation-induced genomic instability in a p53-mutant-type dependent manner at the TCR gene Iwamoto KS,* Seyama T, Mizuno T, Ito T, Tsuyama N, Kyoizumi S, Akiyama M

n In vitro and in vivo oncogene activations by radiation Mizuno T,* Seyama T, Iwamoto KS, Akiyama M

- Somatic cell mutations at the glycophorin A locus in erythrocytes of atomic bomb survivors: Implications for cancer risk Kyoizumi S,* Tanabe K, Cologne JB, Akiyama M

- X-rays induce WAF1/sdi1 accumulation in a dose dependent manner Seyama T,* Tsuyama N, Mizuno T, Iwamoto KS, Kyoizumi S, Akiyama M

- Radiation effects on the immune system in man Akiyama $M$,* Kyoizumi S, Kusunoki Y, Hirai Y

- Incidence of hyperparathyroidism among atomic-bomb survivors in Hiroshima Fujiwara S, * Kodama K, Ezaki H, Nakashima E, Delongchamp RR

- Aortic arch calcification in the atomic bomb survivors, Hiroshima Kasagi F,* Kodama $\mathrm{K}$, Yamada $\mathrm{M}$

- Dose-response among atomic-bomb survivors exposed to low-level radiation Shimizu Y,* Mabuchi K, Preston DL, Pierce DA 
The 11th International Thyroid Congress, 10-15 September 1995, Toronto, Canada

- Association between thyroid autoantibodies and anti-human T-lymphotrophic virus type-I (HTLV-I) antibody in Nagasaki, Japan

Tsuruta M,* Yokoyama N, Shibata Y, Akahoshi M, Matsuo T, Tomonaga M, Izumi $\mathrm{M}$

The 11th Asian-Pacific Congress of Cardiology, 17-22 September 1995, Bali, Indonesia

- The rise and fall of cardiovascular disease in the Asian-Pacific region

Kodama K*

The 40th Meeting of the Japan Society of Human Genetics, 20-22 September 1995, Kumamoto

- Detection of variations in DNA and mRNA by denaturing gradient gel electrophoresis. Report 5. Study of variations observed in various genes

Takahashi N,* Omine H, Miura A, Kaneko J, Satoh C

- Cloning and sequencing of allelic DNA fragments detected by two-dimensional electrophoresis of human genomic DNA

Kodaira M,* Asakawa J, Kuick R, Imanaka M, Kaneoka S, Satoh C

- Effects of atomic-bomb radiation on instability of microsatellites in human germ cells

Satoh C,* Yasunaga K, Miura A

- Identification of a DNA fragment which escaped methylation inactivation on the $\mathrm{X}$ chromosome by two-dimensional electrophoresis of human genomic DNA

Asakawa J,* Kodaira M, Fujiyama A, Kuick R, Kaneoka S, Imanaka M, Neel JV, Hanash SM, Satoh C

The 54th Annual Meeting of the Japanese Cancer Association, 3-5 October 1995, Kyoto

a A novel assay system detecting human somatic mutations at the neutrophil Fcy receptor III locus. II. Study of atomic bomb survivors Hirai Y,* Kusunoki Y, Kyoizumi S, Akiyama M

- Somatic cell mutations at the glycophorin A locus in erythrocyte as a biomarker for cancer risk assessment

Kyoizumi S,* Cologne JB, Hirai Y, Akiyama M

- Evidence for an affect of p53 mutations at the TCR gene loci Iwamoto KS,* Seyama T, Mizuno T, Tsuyama N, Kyoizumi S, Akiyama M

- Early detection of in vivo $T$-cell receptor mutations induced by radiation exposure Ishioka $\mathrm{N},{ }^{*}$ Kyoizumi S, Umeki S, Hirai Y, Ohama K, Akiyama M 
- Analysis of the mechanism of cell cycle checkpoint arrest after X-irradiation Tsuyama N, * Seyama T, Mizuno T, Iwamoto KS, Ide T, Akiyama M

- Molecular analysis of p53 gene mutations in skin cancer among A-bomb survivors

Mizuno T,* Seyama T, Iwamoto KS, Akiyama M, Kishikawa M, Tokunaga M, Tokuoka S, Fujita Y, Mabuchi K

- Ionizing-radiation-induced differentiation of K562 cells Seyama T,* Tsuyama N, Kyoizumi S, Mizuno T, Iwamoto KS, Akiyama M

- Gene rearrangements induced by in-vitro irradiation Seyama T,* Mizuno T, Iwamoto KS, Akiyama M

- Linoleic acid stimulated cell proliferation and inhibited cell-cell communication Hayashi T,* Nomata K, Chang CC, Trosko JE, Akiyama M

- Fifty years after the A-bombings—cancer risk among A-bomb survivors Mabuchi K*

Schull International Symposium, 7-10 October 1995, Houston, Texas

- Biodosimetry of atomic bomb survivors by karyotyping, chromosome painting, and electron spin resonance

Nakamura N,* Awa AA, Pawel DJ, Kodama Y, Nakano M, Ohtaki K, Miyazawa C

- Studies of leukemia risk in the atomic bomb survivors Preston DL*

- Statistical aspects of RERF cancer epidemiology Pierce DA*

The 10th Annual Meeting for the Orthopaedic Research of the Japanese Orthopaedic Association, 12-13 October 1995, Karuizawa

- Epidemiology of osteoporosis

Fujiwara $S^{*}$

The 54th Annual Meeting of Japanese Society of Public Health, 12-14 October 1995, Yamagata

- An attempt to develop markers for evaluation of aging level

Fujita Y,* Kodama K, Kasagi F, Fujita S, Sasaki H, Yanagawa H

The 46th Annual Meeting of the Japanese Electrophoresis Society, 12-13 October 1995, Himeji

- High resolutional two-dimension electrophoresis of DNA: A refinement of RLGS method

Asakawa J* 
The 19th Annual Meeting of Japan Society for Biomedical Gerontology, 18-20

October 1995, Toyonaka

- A-bomb survivors and aging

Kodama K, Kasagi F, Fujiwara S, Yamada M, Neriishi K

Scientific Workshop on Evaluation of Methods for Verification of External Doses of Workers at Mayak Production Association, 31 October-1 November 1995, Chelyabinsk-65, Russia

- ESR dose estimation using tooth enamel from Hiroshima A-bomb survivors Nakamura N,* Miyazawa C, Sawada S, Akiyama M, Awa AA

The 38th Annual Meeting of the Japan Radiation Research Society, 8-10 November 1995, Chiba

- Mitochondrial DNA deletion in human cells exposed to radiation

Ban S, Seyama T, Mizuno T, Iwamoto KS, Tsuyama N, Kyoizumi S, Hirai Y

- Identification of clonal chromosome aberrations by repeated lymphocyte tests in A-bomb survivors

Ohtaki K,* Nakamura N, Awa AA

- ESR dose estimation using tooth enamel from Hiroshima A-bomb survivors. III. Results of 69 survivors for the correlation between stable chromosome aberration frequency vs ESR signal intensity Nakamura N,* Miyazawa C, Sawada S, Akiyama M, Awa AA

- Spontaneous and X-radiation-induced genetic instability at the TCR gene is dependent on the type of $\mathrm{p} 53$ mutation

Iwamoto KS, * Mizuno T, Ito T, Tsuyama N, Kyoizumi S, Akiyama M, Seyama $\mathrm{T}$

- Somatic cell mutations at the glycophorin A locus in erythrocytes of atomic bomb survivors: Implications for cancer risk

Kyoizumi S,* Akiyama M, Cologne JB, Tanabe K, Hirai Y, Umeki S

- Radiation-induced oncogene rearrangements

Mizuno T,* Iwamoto KS, Tsuyama N, Shinohara T, Akiyama M, Seyama T

- Molecular biological analysis of the neutrophil Fcy receptor III gene mutations Hirai Y,* Takahashi K, Kusunoki Y, Akiyama M, Kyoizumi S

Different types of rearrangements in cancer-related genes are inducible by $\mathrm{X}$ irradiation

Seyama $\mathrm{T}^{*}$

- Arteriosclerosis in A-bomb survivors-Analysis of pulse wave velocity Ueda H,* Kodama K, Fujiwara S, Yamada M, Kasagi F, Masunari N

- The mechanism of radiation carcinogenesis as seen from the temporal changes in cancer risk among A-bomb survivors

Mabuchi K,* Pierce DA 
The 10th Meeting of the Japan Menopause Society, 10-11 November 1995,

Fukuoka

- A study on age at menopause in the Nagasaki area (follow-up of a fixed population)

Soda $\mathrm{M}, *$ Akahoshi $\mathrm{M}$

The 5th Asia-Oceania Regional Congress of Gerontology, 19-23 November 1995, Hong Kong

- Epidemiological study of osteoporosis in a Japanese population

Fujiwara $S^{*}$

The 24th Annual Meeting of Japan Environmental Mutagen Society, 20-22 November 1995, Osaka

- Mutant frequency at the Fcy receptor III gene locus of peripheral blood neutrophils of A-bomb survivors

Hirai Y,* Kusunoki Y, Kitahira M, Takahashi K, Ishioka N, Akiyama M, Kyoizumi S

- Monitoring of germ line mutations in A-bomb survivors by DNA-level examination of their children

Satoh C,* Takahashi N, Asakawa J, Kodaira M

International Conference on Health Consequences of the Chernobyl and Other Radiological Accidents, 20-23 November 1995, Geneva, Switzerland

- Profiles of noncancer diseases in the atomic bomb survivors

Kodama K,* Fujiwara S, Yamada M, Kasagi F, Shimizu Y, Shigematsu I

- Studies on germline mutations in the children of atomic bomb survivors Satoh C,* Takahashi N, Asakawa J, Kodaira M

a ER dose estimation using tooth enamel from Hiroshima A-bomb survivors Nakamura N,* Miyazawa C, Sawada S, Akiyama M, Awa AA

- Activated RET oncogene in thyroid cancer among children residing in areas contaminated by the Chernobyl accident and among atomic bomb survivors Seyama T,* Ito T, Iwamoto KS, Mizuno T, Tronko ND, Komissarenko IV, Cherstovoy ED, Takeichi N, Satow Y, Akiyama M

- Mortality study of atomic bomb survivors: Its implications in assessment of radiation accidents

Shimizu Y,* Mabuchi K, Preston DL, Shigematsu I 
The 25th Annual Meeting of the Japanese Society for Immunology, 28-30 November 1995, Fukuoka

- Proliferation in vivo of mutant human T lymphocytes lacking expression of TCR $\alpha \beta$

Kyoizumi S,* Ishioka N, Suzuki T, Akiyama M, Hirai $Y$

- Analysis of multipotential stem cell using a chromosome aberration marker Hirai Y,* Kyoizumi S, Akiyama M, Kusunoki Y

- Analysis of hematopoietic functions using peripheral blood stem cells of A-bomb survivors as indices (1)

Suzuki T,* Hirai Y, Akiyama M, Kyoizumi S

The 4th Japanese General Meeting of Osteoporosis, 30 November-1 December 1995, Hamamatsu

- Relationship between bone mass at middle and old ages and weight and height in early adulthood

Fujiwara S, * Masunari N, Kasagi F, Kodama K, Fukunaga M

The 18th Annual Meeting of the Molecular Biology Society of Japan, 6-9 December 1995, Nagoya

- Cloning and sequencing of the DNA spots specific for a lung tumor identified by two-dimensional electrophoresis of DNA

Kodaira M,* Asakawa J, Hiyama K, Imanaka M, Kaneoka S, Tsuji T, Satoh C, Yamakido $M$

- Study on a DNA fragment which escaped methylation inactivation of the X chromosome identified by two-dimensional electrophoresis of human DNA

Asakawa J,* Kodaira M, Fujiyama A, Kuick R, Kaneoka S, Imanaka M, Neel JV, Hanash SM, Satoh C

The 4th Meeting of Metabolic Bone Diseases, 9 March 1996, Tokyo

n Risk factors of hip fracture using cohort studies

Fujiwara S, * Kasagi F, Yamada M, Kodama K

The 36th Annual Conference on Cardiovascular Disease Epidemiology and Prevention, 13-16 March 1996, San Francisco, California

- Physical activity at work and at leisure and 20-year total and noncancer mortality in Japanese men in Japan and Hawaii

Curb JD, * Kodama K, Rodriguez B, Abbott R, Kasagi F

- Diabetes, impaired glucose tolerance and 20 -year total and noncancer mortality in Japanese men in Japan and Hawaii

Curb JD, * Kodama K, Rodriguez B, Abbott R, Kasagi F 
The 7th Symposium of the Nuclear Safety Research Association, 15 March 1996, Tokyo

- Radiation risk estimation by somatic mutation Kyoizumi S*

- Epidemiology and dose assessment Nakamura $\mathrm{N}^{*}$

\section{Human Genome Meeting 1996, 22-24 March 1996, Heidelberg, Germany}

- Identification and molecular analysis of a novel locus on the X chromosome which escapes methylation inactivation

Asakawa J,* Kodaira M, Kuick R, Glover TW, Neel JV, Fujiyama A, Yoshikawa $\mathrm{H}$, Satoh C, Hanash SM 


\section{Scientific Lectures and Seminars}

\section{April 1995-31 March 1996}

Klaus Becker, executive secretary, DIN German Nuclear Standards Committee; secretary, ISO/TC 85 "Nuclear Energy", Berlin, Germany: "Radiation protection standards and their implication: Some reflections from Europe," 17 April

Megu Ohtaki, associate professor, Department of Environmetrics and Biometrics, Research Institute for Radiation Biology and Medicine, Hiroshima University. 143rd Hiroshima Joint Statistics Seminar: "Mathematical implication for radiation carcinogenesis," 28 April

Sheldon Wolff, director, Laboratory of Radiobiology and Environmental Health, University of California, San Francisco: "Newer cytogenetic studies on the adaptive response to low doses of radiation" and "A novel way to study SCEs by using endoreduplicated cells," 22 May

Jun-ichiro Fukuchi, lecturer, Department of Economics, Hiroshima University. 144th Hiroshima Joint Statistics Seminar: "Bootstrapping extremes and its applications," 26 May

Fumiyoshi Kasagi, research scientist, Department of Statistics, RERF. 145th Hiroshima Joint Statistics Seminar: "Effects of nonparticipation on a dose response association observed among participants," 30 June

Alex $\mathbf{J}$ van der $\mathbf{E b}$, professor of molecular carcinogenesis, Faculty of Medicine, Department of Medical Biochemistry, Section of Molecular Carcinogenesis, Medical Genetics Centre, University of Leiden, The Netherlands: "Radiation-induced stress responses and cancer-proneness," 18 September

Shizue Izumi, research assistant, Department of Statistics, RERF. 146th Hiroshima Joint Statistics Seminar: "Size of the modified Ansari-Bradley test," 22 September

Teruo Fujioka, research assistant, Department of Mathematics, Faculty of Science, Hiroshima University. 147th Hiroshima Joint Statistics Seminar: "An overview of testing goodness of fit of a linear model in nonparametric regression," 20 October

CB Meinhold, IRPA Chairman, ICRP Main Commission Vice Chairman, NCRP President: "The basis for the dose limits," 5 December 
Robert R Delongchamp, research scientist, Department of Statistics, RERF. 148th Hiroshima Joint Statistics Seminar: "Cancer mortality among A-bomb survivors with in utero or childhood exposures," 8 December

Elisabeth Cardis, head, Programme on Radiation and Cancer, International Agency for Research on Cancer: "Cancer mortality among nuclear industry workers in Canada, the United Kingdom and the United States of America," 11 December

Yasunori Fujikoshi, professor, Department of Mathematics, Faculty of Science, Hiroshima University. 149th Hiroshima Joint Statistics Seminar: "Selection of variables in some multivariate models-new corrections of AIC and MIC," 19 January

Tsunehiro Mukai, chief, Department of Bioscience, National Cardiovascular Center Research Institute: "Genomic imprinting," 14 February 


\section{Periodicals Produced by RERF}

During FY95, the following periodicals were produced by the RERF Publication and Documentation Center to provide various information including RERF research findings for the local scientific community, and for members of the national and international radiation-research community and for RERF employees-past and present.

\section{Journal of the Hiroshima Medical Association}

Since 1960, the monthly Journal of the Hiroshima Medical Association (JHMA) has included a section devoted to ABCC-RERF research findings. During FY95, original English-language articles written for RERF Update have been translated into Japanese for this purpose. In addition, bibliographic information and the Scientific Council report have been published. Page charges are contributed by RERF to disseminate this material to the 5500 members of the local medical association and medical libraries and medical institutions throughout Japan. In addition, 70 reprints are purchased and distributed by RERF to a mailing list of 51 persons, local media and medical institutions.

\section{RERF Update}

Since 1989, RERF has published a quarterly English-language newsletter, RERF Update, to inform international radiation researchers about RERF's recent journal publications, about institutional news, and about ongoing scientific projects. For various reasons, one issue of RERF Update was published during FY95 and distributed to 957 persons or institutions free of charge.

\section{RERF Newsletter}

Since 1975, the monthly RERF Newsletter has been published in Japanese to disseminate institutional, scientific and employee news.

From the issue of May 1995, the RERF Newsletter became a bimonthly publication. During the year, six issues were published in odd number months and a special issue was published on the Twenty-ninth Board of Directors Meeting and distributed to 755 persons or institutions free of charge. Employee news was distributed as inserts in the newsletter confined to 505 present and past RERF employees.

\section{RERF Annual Report}

Based on the response when asked to indicate if they wished to continue receiving the RERF Annual Report , 199 copies of the Japanese-language version and 223 copies of the English-language version were distributed free of charge to individuals and institutions, including 130 libraries. 


\section{RERF Reports}

After the termination of the in-house RERF Technical Report Series in 1992, RERF began to purchase journal-article reprints, which are bound into RERF Report covers with Japanese summaries. Although distribution of RERF Reports varies depending on the subject matter, at least 100 are disseminated to Japanese and American governmental ministries and agencies, local hospitals, A-bomb survivor groups, libraries and RERF directors and consultants.

In addition, about 500 reprints of RERF Report were sent to international researchers in response to their requests.

Requests for RERF journal-article reprints by country, April 1995-March 1996

\begin{tabular}{|c|c|c|c|c|c|}
\hline Country & $\begin{array}{l}\text { No. of } \\
\text { requests }\end{array}$ & Country & $\begin{array}{l}\text { No. of } \\
\text { requests }\end{array}$ & Country & $\begin{array}{l}\text { No. of } \\
\text { requests }\end{array}$ \\
\hline USA & 130 & Czech Republic & 6 & Ecuador & 1 \\
\hline Japan & 71 & Lithuania & 6 & Estonia & 1 \\
\hline France & 34 & Slovakia & 6 & Finland & 1 \\
\hline Poland & 33 & United Kingdom & 6 & Indonesia & 1 \\
\hline Germany & 31 & Argentina & 5 & Ireland & 1 \\
\hline India & 22 & Brazil & 4 & Luxembourg & 1 \\
\hline South Africa & 20 & Croatia & 4 & Macedonia & 1 \\
\hline Hungary & 12 & Malaysia & 3 & Portugal & 1 \\
\hline Norway & 11 & Switzerland & 3 & Romania & 1 \\
\hline Bulgaria & 10 & Ukraine & 3 & Russia & 1 \\
\hline Canada & 9 & Yugoslavia & 3 & South Korea & 1 \\
\hline Spain & 9 & Chile & 2 & Sweden & 1 \\
\hline The Netherlands & 9 & Cuba & 2 & Syria & 1 \\
\hline Belgium & 7 & Italy & 2 & Turkey & 1 \\
\hline Israel & 7 & Australia & 1 & & \\
\hline Austria & 6 & Costa Rica & 1 & & \\
\hline
\end{tabular}




\section{International Collaborative Activities}

Editor's note: This listing does not include participation in international scientific meetings. For a list of papers delivered at national and international meetings, see $p$ 87. Also, see the Appendix, $p$ 176, for agreements on RERF international collaborations.

\section{Participation in international collaborative activities by RERF directors and staff members}

\section{Chernobyl-related collaborative activities}

- Sasakawa Memorial Health Foundation

1. Yoshisada Shibata, chief, Department of Epidemiology and Biometrics (Nagasaki), provided on-site technical guidance to the Chernobyl Medical Cooperation Project. (Ukraine, May 1995)

2. Yoshisada Shibata, chief, Department of Epidemiology and Biometrics (Nagasaki), participated in the workshop of the Chernobyl Medical Cooperation Project. (Russia, July 1995)

3. Yoshisada Shibata, chief, Department of Epidemiology and Biometrics (Nagasaki), provided on-site technical guidance to the Chernobyl Medical Cooperation Project. (Russia, Ukraine, Belarus, September-October 1995)

4. RERF Chairman Itsuzo Shigematsu and Yoshisada Shibata, chief, Department of Epidemiology and Biometrics (Nagasaki), participated in the Fourth Chernobyl Medical Cooperation Symposium. Shibata also provided on-site technical guidance to the Chernobyl Medical Cooperation Project. (Belarus, Russia, November 1995)

5. Tomoko Motomura, acting chief, Division of Clinical Laboratories, Department of Clinical Studies (Nagasaki), provided on-site technical guidance to the Chernobyl Medical Cooperation Project. (Russia, Belarus, March 1996)

\section{- World Health Organization (WHO)}

1. Shizuyo Kusumi, assistant chief, Department of Clinical Studies, and Kazuo Neriishi, chief, Division of Medicine, Department of Clinical Studies, participated in the international meeting on Chernobyl medical cooperation. (Ukraine, May 1995)

2. Tomoko Nishikawa, acting chief, Division of Clinical Laboratories, Department of Clinical Studies (Nagasaki), participated in the Chernobyl Medical Cooperation Project meeting. (Belarus, July 1995) 
3. RERF Chairman Itsuzo Shigematsu, Yoshisada Shibata, chief, Department of Epidemiology and Biometrics (Nagasaki), Kazunori Kodama, chief, Department of Clinical Studies, Chiyoko Satoh, chief, Department of Genetics, Nori Nakamura, assistant chief, Department of Genetics, Toshio Seyama, assistant chief, Department of Radiobiology, and Yukiko Shimizu, assistant chief, Department of Epidemiology, participated in the international conference on health effects of radiation exposure in Geneva. (Switzerland, November 1995)

- International Atomic Energy Agency (IAEA)

Toshio Seyama, assistant chief, Department of Radiobiology, participated in the seminar on children affected by the Chernobyl accident held in Havana. (Cuba, November 1995)

\section{- Japanese Ministry of Foreign Affairs}

Yoshisada Shibata, chief, Department of Epidemiology and Biometrics (Nagasaki), and Hironori Ueda, research scientist, Department of Clinical Studies, conducted onsite investigation related to the Chernobyl nuclear accident as a member of the dataprocessing group. (December 1995)

\section{Chelyabinsk-related cooperation}

- US National Cancer Institute (NCI)

1. Dale L Preston, chief, Department of Statistics, participated in the international joint workshop on radiation effects. (Russia, July 1995)

2. Dale L Preston, chief, Department of Statistics, participated in the joint research project meetings of NCI-RERF-Branch N1, Institute of Biophysics (FIB-1) and NCI-RERF-Urals Research Center for Radiation Medicine (URCRM). (Russia, October-November 1995.)

\section{- Japan Atomic Energy Research Commission}

Nori Nakamura, assistant chief, Department of Genetics, participated in the workshop on external exposure doses of decontamination workers at Mayak. (Russia, OctoberNovember 1995)

\section{Other international collaborative activities}

- Medical examination of A-bomb survivors in North America (Japan Public Health Association, Hiroshima Prefectural Medical Association, Hiroshima Prefecture and City, Hiroshima A-bomb Casualty Council, RERF)

Kazuo Neriishi, chief, Division of Medicine, Department of Clinical Studies, and Tadaaki Watanabe, supervisor, General Affairs Unit, General Affairs Section, 
Secretariat, participated in the tenth medical examination of A-bomb survivors in North America. (US, June-July 1995)

\section{- International Commission on Radiological Protection (ICRP)}

Kiyohiko Mabuchi, chief, Department of Epidemiology, participated in the ICRP joint conference. (Germany, September, 1995)

- Hiroshima International Council for Health Care of the Radiation-exposed (HICARE)

1. RERF Chairman Itsuzo Shigematsu exchanged opinions with Hawaii Medical Association members, and reported on on-site investigation and HICARE activities. (US, October 1995)

2. Yasuyuki Fujita, assistant chief, Department of Epidemiology, participated in the International Tumor Registry Conference in Goiania, visited the Leide das Neves Ferreira Foundation, exchanged opinions on studies concerning the radiation accident in Goiania, and reported on HICARE activities. (Brazil, October-November 1995)

\section{Acceptance of visitors from overseas for briefing and training (grand total: 287 persons)}

\section{Hiroshima Laboratory: 260 persons}

Chernobyl-related trainees: 24 persons (all accepted through HICARE) 1. Japanese Red Cross Society. Three doctors from Minsk, the Republic of Belarus, Moscow, the Russian Federation, and Kiev, Ukraine from 27 July to 1 August, and three doctors from Moscow, the Russian Federation, Minsk, the Republic of Belarus, and Kiev, Ukraine from 25 to 27 March 1996.

2. HICARE. Two doctors from Minsk, the Republic of Belarus from 31 July to 4 August, one doctor from Kiev, Ukraine from 23 to 25 October, and one doctor from Riga, the Republic of Latvia from 23 to 27 October.

3. National Institute of Radiological Sciences. Two doctors from Moscow, the Russian Federation on 15 November.

4. Japanese Ministry of Foreign Affairs. Two leukemia specialists from Vitebsk, the Republic of Belarus from 29 to 31 January 1996, seven data-processing specialists from Obninsk and St Petersburg, the Russian Federation; Minsk, the Republic of Belarus; Kiev, Ukraine; Vilnius, the Republic of Lithuania; Riga, the Republic of Latvia; and Tallin, the Republic of Estonia from 4 to 7 March, three radiobiologists 
from Vilnius, the Republic of Lithuania; Riga, the Republic of Latvia; and Tallin, the Republic of Estonia from 4 to 13 March.

Chelyabinsk-related trainees: 14 persons (accepted through HICARE) 1. RERF-Branch N1, Institute of Biophysics (FIB-1) collaboration agreement. One specialist from Chelyabinsk, the Russian Federation from 17 April to 10 May and from 17 April to 24 May, two specialists from Chelyabinsk and one from Moscow, the Russian Federation from 22 to 24 May.

2. RERF-Urals Research Center for Radiation Medicine (URCRM) collaboration agreement. Two specialists from Ekaterinburg and one doctor from Kurgan, the Russian Federation from 16 to 23 May, and one specialist from Chelyabinsk, the Russian Federation from 18 July to 17 August.

3. Marubeni Utility Services, Ltd. Four specialists from Chelyabinsk and one specialist from Moscow, the Russian Federation on 24 July.

\section{Other HICARE-related trainees: 15 persons}

1. San Diego County Medical Society. One doctor from San Diego, California on 4 April.

2. Hawaii Medical Association. One doctor from Honolulu, Hawaii from 10 May to 2 June.

3. National Atomic Energy Commission, Argentina. One specialist from Buenos Aires, Argentina from 16 June to 14 July.

4. US National Cancer Institute. One specialist from Bethesda, Maryland from 21 June to 7 July.

5. King County Medical Society. One doctor from Seattle, Washington from 1 to 2 November.

6. Hiroshima Peace Culture Foundation. Delegation of the "Chinese People's Association for Peace and Disarmament" (five members) from Beijing, the People's Republic of China on 8 December.

7. Faculty of Medicine, Osaka University. Five trainees from the "Course of Advanced Medical Radiological Technology, FY95" from Central and South American, African and Southeast Asian countries on 7 March 1996. 


\section{Japan International Cooperation Agency (JICA)-related trainees: 42 persons}

1. Research Institute of Tuberculosis, Japan Anti-tuberculosis Association. Fourteen trainees from the "Group Training Course in Tuberculosis Control for Administrative Medical Officers" from Southeast Asian, African, and Central and South American countries on 30 May.

2. Japan Chemical Analysis Center. Four trainees from the "Course of Environmental Radioactivity Analysis and Measurement" from Mongolia, the Republic of Ghana, Thailand and the Philippines on 6 October.

3. National Cancer Center Research Institute. Thirteen trainees from the "Course of Clinical Oncology" from Central and South American, Southeast Asian and African countries on 19 October.

4. National Cardiovascular Center Research Institute. Six trainees from the "Cardiovascular Diseases Course, FY95" from Central and South American, African and East European countries on 9 November.

5. Faculty of Medicine, Osaka University (same as 7 above). Five trainees from the "Course of Advanced Medical Radiological Technology, FY95" from Central and South American, African and Southeast Asian countries on 7 March 1996.

\section{Other visitors: 170 persons}

1. Nissho Iwai, Inc. Five specialists from the Russian Federation on 18 May.

2. National Yang Ming University Medical School, Graduate Institute of Public Health. Two specialists from Taiwan on 3 July.

3. International Physicians for the Prevention of Nuclear War (IPPNW). Two doctors from the People's Republic of China and three doctors and others from the Democratic People's Republic of Korea on $10 \mathrm{July.}$

4. Hiroshima International Cultural Foundation. Five reporters from the "Asian Reporters Invitation Project" from Vietnam, Israel, the Republic of Korea and Hong Kong on 4 August.

5. Hiroshima Peace Culture Foundation. One doctor from the US on 7 August. 
6. Peace Memorial Ceremony participation project sponsored by Hiroshima Prefecture and City. Nine A-bomb survivors residing in North and South America on 9 August.

7. Japan Center for International Exchange (JCIE/JAPAN). Delegation of the "IsraelJapan Intellectual Exchange Program (thirteen persons)" from Israel on 14 September.

8. National Institute of Radiological Sciences. Two specialists from Rome, Italy on 28 March 1996.

9. Others. Researchers, doctors and others visited the RERF individually.

\section{Nagasaki Laboratory: 27 persons}

Chernobyl-related trainees: 11 persons (all accepted through Nagasaki Association for Hibakushas' Medical Care-NASHIM)

1. NASHIM. Four specialists from Klintsy, the Russian Federation; Gomel and Mogilyov, the Republic of Belarus; and Kiev, Ukraine on 25 August.

2. Japanese Ministry of Foreign Affairs. Seven data-processing specialists from Obninsk and St Petersburg, the Russian Federation; Minsk, the Republic of Belarus; Kiev, Ukraine; Vilnius, the Republic of Lithuania; Riga, the Republic of Latvia; and Tallin, the Republic of Estonia on 11 March, 1996.

\section{Other NASHIM-related trainee: 1 person}

Republic of Korea Red Cross Society. One doctor from Seoul, the Republic of Korea on 28 August.

\section{Japan International Cooperation Agency (JICA)-related trainees: 15 persons}

1. National Institute of Radiological Sciences. Nine trainees from Southeast Asian countries enrolled in the "Course of Advanced Medical Radiological Technology, FY95" on 3 September.

2. Okinawa Prefectural Institute of Health and Environment. Six trainees from Central and South American, African and Southeast Asian countries enrolled in the "Public Hygiene and Environmental Contamination Analysis Course for FY95" on 9 November. 


\section{International meeting}

\section{Sixth WHO Meeting on Radiation Emergency Medical Preparedness and Assistance Network (REMPAN)}

For three days from October 23 to 25, 1995, the sixth REMPAN meeting of WHO was held at RERF's Hiroshima Laboratory Auditorium.

In March 1987, approximately a year after the catastrophic Chernobyl nuclear power plant accident, WHO, feeling the need of a system for medical emergency measures and speedy assistance in case of such accidents, called on WHO's designated collaborative research institutions in the area of radiation medicine and held the first meeting to explore ways to establish a network for such emergency measures and assistance. Ever since, five meetings were held as of 1995.

The RERF, a WHO collaborative research center on the effects of radiation on man, has been invited and is participating in the network meetings from the second meeting in 1988. In commemoration of the 50th anniversary of the atomic bombing and the 20th anniversary of the foundation of RERF, the RERF invited the sixth meeting to be held at its Hiroshima Laboratory.

The meeting opened with an address by Wilfried Kreisel, followed by speeches of welcome by Chairman Shigematsu, Hiroshima Vice Governor Nobuyasu Kubo and Hiroshima Mayor Takashi Hiraoka. Special reports were presented on the emergency measures of such international organizations as WHO and IAEA (International Atomic Energy Agency); on the estimated proliferation of radioactive substances when a nuclear power plant accident occurs, on the No. 5 Lucky Dragon incident, on the iridium exposure accident in Chiba, on the health effects of the nuclear arms plant accident in south Ural, and on the radiation accident in Estonia.

Three panel discussions were held. First, the representatives of WHO collaborative research centers of nine countries reported on the activities of their respective centers after the last meeting (December last year). They also explained the radiation emergency measures of their respective countries. Four panelists reported on "acute disturbances induced by radiation exposure and their treatment," the theme of the second panel discussion. The third panel discussion was on the follow-up and registration of patients and creation of a database for the collected information. Kyyohiko Mabuchi, chief, Department of Epidemiology, explained about the follow-up study underway at RERF.

Lastly, Dr Kreisel, executive director of WHO, presented WHO's view of the goal of the network and the operation plan to attain it. The next meeting will be held in Brazil in 1997. 


\section{Ministry of Health and Welfare-entrusted Database Development}

RERF, entrusted by the Japanese Ministry of Health and Welfare (JMHW), has organized the "Committee on A-bomb Materials and Information Network (Chairman: Professor Emeritus Yasuo Yoshizawa, Tokyo University)" and worked on developing an A-bomb-related materials database system since FY1991.

JMHW intends to build the "A-bomb Dead Memorial and Peace Praying Hall" in Hiroshima in FY1998 and in Nagasaki in FY1999 and situate the database system in the halls.

The said committee has developed a draft of WWW home page last fiscal year to introduce and publicize the activities of the halls through the Internet.

\section{0th Anniversary of the Establishment of RERF}

In commemoration of the 20th anniversary of RERF, which is funded equally by Japan and the United States based on the official documents exchanged between the two countries, ceremonies and commemorative lectures were held in Hiroshima and Nagasaki on 28 and 29 June 1995. Subsequently, the open house of the Hiroshima laboratory was held on 5 August.

\section{Commemoration ceremony and lectures}

Hiroshima, 28 June 1995

Minami-ward Culture Center Hall

Ceremony:

Overall moderator

Opening remarks

Greetings

Addresses by guests

\section{Lectures:}

Moderator

Rumi Tsuchioka, Accounting Section, Secretariat

Yutaka Hasegawa, permanent director

Itsuzo Shigematsu, chairman

Manzo Hamamoto, minister of labor

Akihito Matsumura, director-general, Health Service

Bureau, Ministry of Health and Welfare (MHW)

Paul Seligman, deputy assistant secretary for health,

Department of Energy (DOE)

Donald R Harkness, permanent director (chief of research) 
"International Role of RERF," Warren K Sinclair, president emeritus, US National Council on Radiation Protection and Measurement

"In Retrospect of 50 years since A-bombings," Toshiyuki Kumatori, chairman, Radiation Effects Association

Closing remarks

Yasukiyo Hirano, chief of secretariat

Reception

RERF dining hall

\section{Nagasaki, 29 June 1995}

Nagasaki Town and Village Hall

\section{Ceremony:}

Overall moderator

Opening remarks

Greetings

Addresses by guests

Lectures:

Reception
Takaaki Hashiguchi, chief, General Affairs Section Yutaka Hasegawa, permanent director Itsuzo Shigematsu, chairman Akihito Matsumura, director-general, Health Service Bureau, MHW

Paul Seligman, deputy assistant secretary for health, DOE The same as in Hiroshima.

RERF

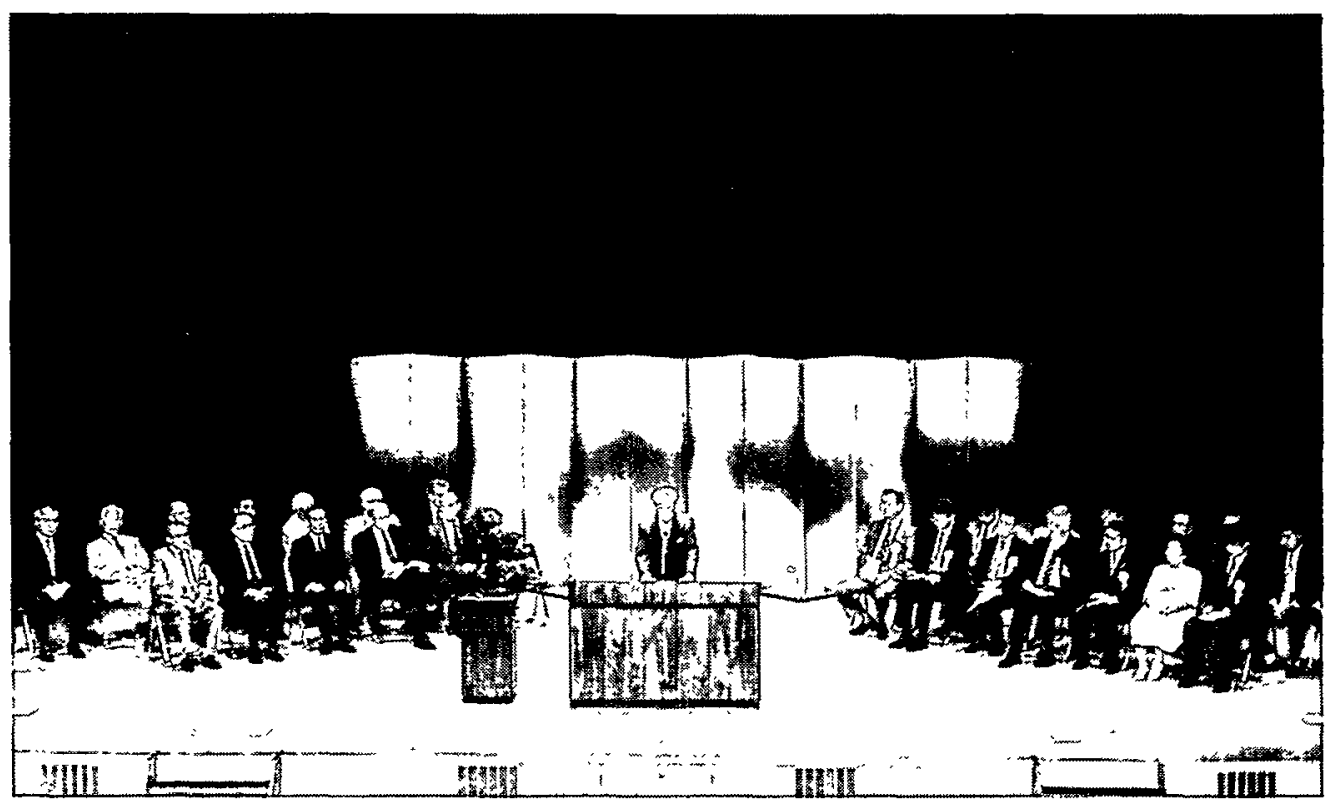

Ceremony for the 20th RERF Anniversary at the Minami-ward Culture Center Hall. 


\section{Guests}

MHW:

Akihito Matsumura, director-general, Health Service Bureau

Hiroshi Maruyama, assistant director, Planning Division, Health Service Bureau

Gojo Wakamatsu, section chief, General Affairs Section, Planning Division, Health Service Bureau

\section{DOE:}

Paul Seligman, deputy assistant secretary for health

Milton Eaton, representative of DOE, US Embassy

\section{US National Academy of Sciences:}

John D Zimbrick, director, Board on Radiation Effects Research, Commission on Life Sciences, National Research Council

Catherine S Berkley, administrative associate, Commission on Life Sciences, National Research Council

\section{Local government/organization:}

Keiichi Nakabayashi, chairman, Welfare and Health Affairs Department, Nagasaki Prefecture

Isamu Kobada, chief, Atomic Bomb Victims Affairs Division, Welfare and Health Affairs Department, Nagasaki Prefecture

\section{Labor union supporters:}

Kenji Taguchi, member of the House of Representatives, National Diet Advisory Group for RERF Labor Union

Masatsugu Kato, executive chairman, Nagasaki Prefecture Headquarters, All Japan Prefectural and Municipal Workers' Union

\section{Open house}

The RERF laboratory in Hiroshima was opened to the public for the first time in the history of ABCC-RERF on 5 August. Close to 900 visitors turned out for the open house.

Many of them crowded in front of the panels prepared by each department and the demonstration booths of the latest technology used for research and studies also attracted attention throughout the day. 


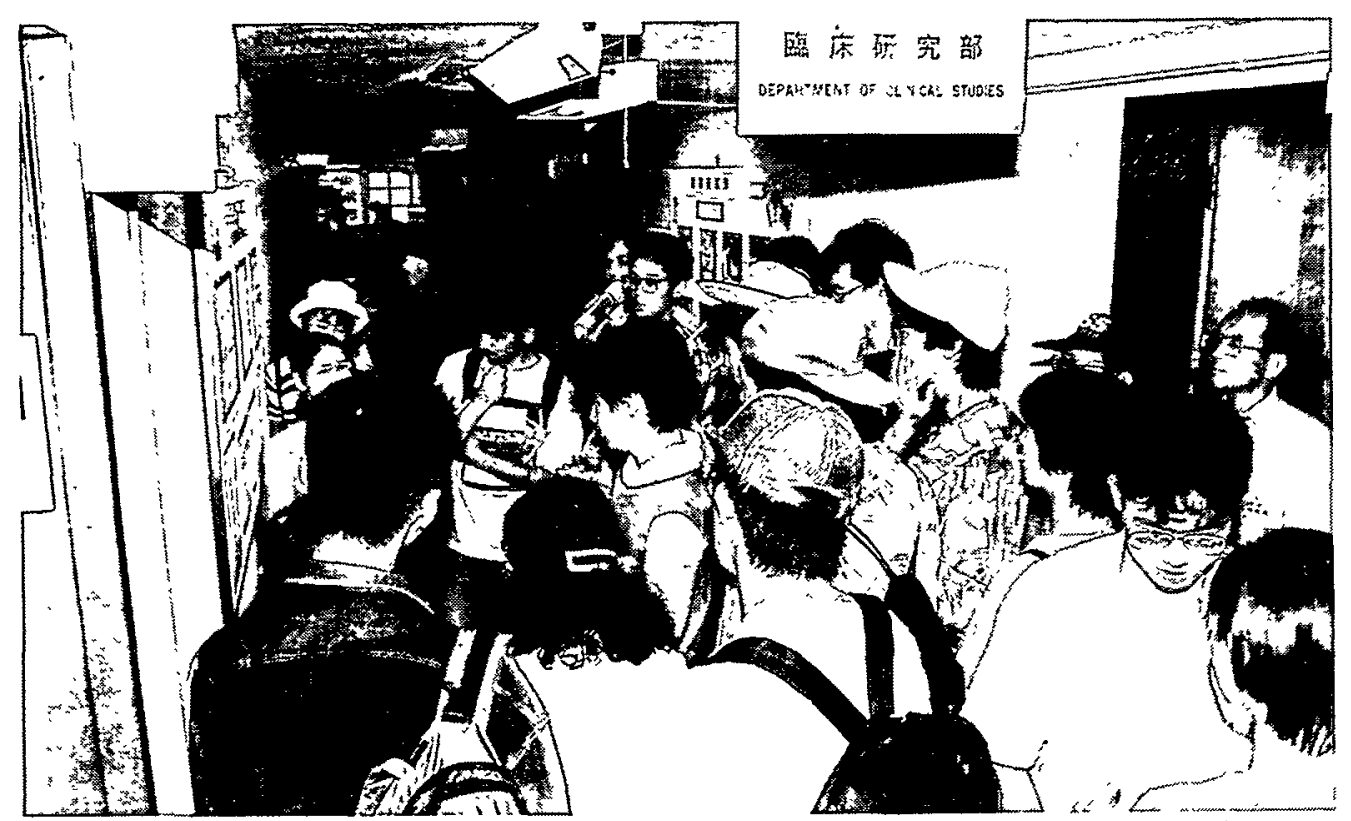

Visitors listen to the explanation of the panels prepared by the Department of Clinical Studies.

\section{Visitors to RERF}

Beginning in April 1995 through March 1996, a total of 1,112 trainees, medicalrelated persons, and visitors from abroad who were accepted through international exchange organizations, as well as government officials, medical-related persons, students, and members of citizen's groups from inside Japan visited RERF. The number of visitors came up to 2,009 including 897 visitors to the first Hiroshima Laboratory open house on 5 August, held as an 50th RERF anniversary event.

\section{Commendations}

At a general conference of International Higher Education Academy of Sciences held in Moscow, Chairman Shigematsu was selected honorary academician at the recommendation of Anatoly Yc Romanenko, president of the Ukraine Research Center for Radiation Medicine, in recognition of his contributions of a global level to radiological research and to the medical investigation following the Chernobyl nuclear power plant accident in particular.

Headquartered in Moscow, International Higher Education Academy of Sciences registers a total membership of 375 scientists from 27 countries. 


\section{In memoriam: H Grant Taylor}

H Grant Taylor, who had served with distinction as deputy medical director for research with the Atomic Bomb Casualty Commission from 1949 to 1953 and later as its director, passed away at his home in Houston, Texas on 19 September 1995. He was 92 years old.

Taylor was a pediatrician by profession, chaired the Department of Pediatrics of the MD Anderson Cancer Center, University of Texas for many years and later served as director of continuing education. He was instrumental in the introduction of the multi-drug treatment of childhood cancers and was responsible for the

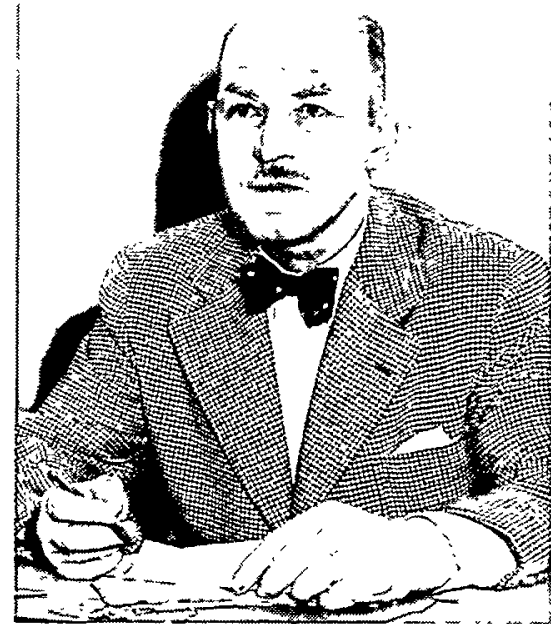

Taylor at his ABCC days training of a large number of Japanese physicians in cancer therapy. 


\section{Report from the Secretariat}

\section{9th Board of Directors Meeting}

The 29th meeting of the Board of Directors was held at the Hiroshima Laboratory, 26-28 June. At the meeting, the recommendations of the 22 nd meeting of the Scientific Council (19-21 April 1995) were carefully discussed as one of the most important items for deliberation, and they were officially adopted by the Board of Directors. While Japan-US talks on the budget issue were in progress, the DOE presented its plans to transfer US side management of the RERF from the US National Academy of Sciences (NAS) to some other organization (DOE had a university in mind), however, it eventually was decided to extend the relationship of NAS to RERF for two years, and, in the meantime, to have RERF's current and future scientific activities assessed by an international committee of distinguished scientists ("Blue Ribbon Panel") as recommended by the Scientific Council. (The report on the 29th Board of Directors meeting begins in the appendix on page 129.)

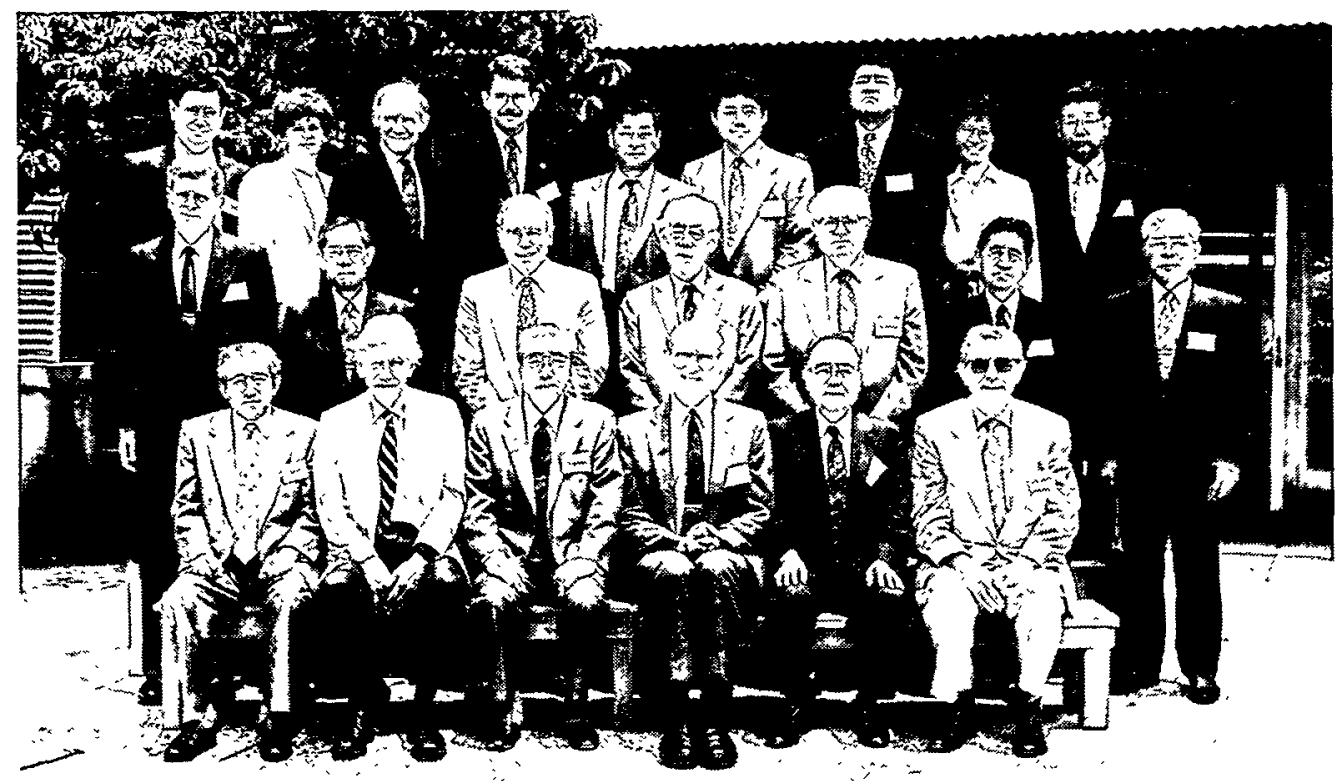

Attendees of the 29th RERF Board of Directors meeting included, front row from left, T Kumatori, J Rall, I Shigematsu, ML Mendelsohn, K Arichi, W Sinclair. Middle row from left, RD Sperry. Y Hasegawa, RD Harkness, E Matsunaga, S Jablon, A Shishido, Y Hirano. Back row from left, J Zimbrick, $C$ Berkley, $D$ Williams, P Seligman, T Nakaoka, H Maruyama, T Fujitani, JL ohara, A Awa. 


\section{Personnel}

RERF employees totaled 336 as of 31 March 1996, a decrease of 41 compared to a year ago. Because of the policy of gradually reducing budgeted positions, employment of new general employees is being kept to a minimum. (See Tables 1 and 2.)

Table 1a. Full-time personnel as of 31 March 1996

\begin{tabular}{lrrrrr}
\hline Full-time personnel & \multicolumn{2}{c}{ Hiroshima } & Nagasaki & \multicolumn{2}{c}{ Total } \\
\hline Directors & 4 & $(1)$ & & 4 & $(1)$ \\
Professionals* & 39 & $(8)$ & 6 & 45 & $(8)$ \\
General & 213 & $(1)$ & 74 & 287 & $(1)$ \\
Total & 256 & $(10)$ & 80 & $336 \quad(10)$ \\
\hline
\end{tabular}

*Numbers in parentheses are personnel recruited by US National Academy of

Sciences.

Table 1b. Other RERF personnel

\begin{tabular}{|c|c|c|c|c|c|c|}
\hline \multirow[b]{2}{*}{ Department/division } & \multirow{2}{*}{$\begin{array}{l}\text { Consul- } \\
\text { tants* }\end{array}$} & \multirow{2}{*}{$\begin{array}{l}\text { Expert } \\
\text { advisors* }\end{array}$} & \multicolumn{3}{|c|}{ Part-time professionals } & \multirow[b]{2}{*}{ Total } \\
\hline & & & $\mathrm{H}$ & $\mathrm{N}$ & Total & \\
\hline Clinical Studies & 30 & 7 & 10 & 11 & 21 & 58 \\
\hline Genetics & 7 & 1 & 2 & 0 & 2 & 10 \\
\hline Radiobiology & 6 & 0 & 1 & 1 & 2 & 8 \\
\hline Epidemiology & 17 & 14 & 2 & 2 & 4 & 35 \\
\hline Statistics & 4 & 3 & 1 & 0 & 1 & 8 \\
\hline Information Technology & 3 & 1 & 0 & 0 & 0 & 4 \\
\hline Secretariat & 2 & 0 & 1 & 0 & 1 & 3 \\
\hline Total & 69 & 26 & 17 & 14 & 31 & 126 \\
\hline
\end{tabular}

*See Appendix, pp 158-163, for the list of consultants and expert advisors.

Note: $\mathrm{H}=$ Hiroshima. $\mathrm{N}=$ Nagasaki. 
Table 2. Personnel composition

As of 31 March 1996

\begin{tabular}{|c|c|c|c|c|c|c|c|c|c|c|}
\hline & Department or section & Directors & $\begin{array}{l}\text { Research } \\
\text { scientists }\end{array}$ & $\begin{array}{l}\text { Admini- } \\
\text { strative }\end{array}$ & $\begin{array}{l}\text { Manual \& } \\
\text { gen. tech. }\end{array}$ & $\begin{array}{l}\text { Medical \& } \\
\text { technical (I) }\end{array}$ & $\begin{array}{c}\text { Medical \& } \\
\text { technical (II) }\end{array}$ & Total & $\begin{array}{l}\text { Visiting } \\
\text { scientists }\end{array}$ & $\begin{array}{l}\text { Temporary } \\
\text { employees* }\end{array}$ \\
\hline 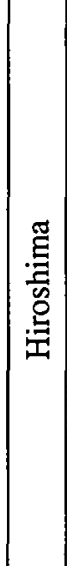 & $\begin{array}{l}\text { Directors } \\
\text { Chief of Secretariat } \\
\text { Clinical Studies } \\
\text { Genetics } \\
\text { Radiobiology } \\
\text { Epidemiology } \\
\text { Statistics } \\
\text { Information Technology } \\
\text { Publication \& Docum. Ctr } \\
\text { Radioisotope Facility } \\
\text { Secretariat Total }\end{array}$ & $\begin{array}{l}3 \\
1\end{array}$ & $\begin{array}{r}6 \\
8 \\
10 \\
4 \\
8 \\
2 \\
1\end{array}$ & $\begin{array}{r}19 \\
3 \\
5 \\
36 \\
4 \\
17 \\
20 \\
\\
39 \\
143\end{array}$ & $\begin{array}{l}1 \\
2 \\
2\end{array}$ & $\begin{array}{r}10 \\
16 \\
14 \\
2\end{array}$ & 13 & $\begin{array}{r}3 \\
1 \\
49 \\
29 \\
31 \\
42 \\
12 \\
19 \\
21 \\
1 \\
48 \\
256\end{array}$ & $\begin{array}{l}1 \\
1 \\
2 \\
\\
4\end{array}$ & \\
\hline 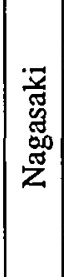 & \begin{tabular}{l} 
Clinical Studies \\
Radiobiology \\
Epidemiology \\
Radioisotope Facility \\
Secretariat \\
\multicolumn{1}{|c|}{ Total }
\end{tabular} & & $\begin{array}{l}2 \\
2 \\
2 \\
6\end{array}$ & $\begin{array}{r}15 \\
1 \\
17 \\
16 \\
49\end{array}$ & $\begin{array}{l}1 \\
2\end{array}$ & $\begin{array}{c}9 \\
3 \\
3 \\
1 \\
16\end{array}$ & $\begin{array}{l}7 \\
7\end{array}$ & $\begin{array}{r}34 \\
6 \\
22 \\
1 \\
17 \\
80\end{array}$ & & $\begin{array}{l}3 \\
3\end{array}$ \\
\hline & Grand total & 4 & 45 & 192 & 16 & 59 & 20 & 336 & 4 & 3 \\
\hline
\end{tabular}

* Supported by entrusted funds.

Note: 1 . Among the 192 administrative and clerical staff, 137 are in research support units and 55 are in the Secretariat.

2. Four temporary employees (substitutes for employees on child care leave, etc) are not included here. 


\section{Change of director}

Seymour Abrahamson was elected as successor to Vice Chairman Mortimer L Mendelsohn, who resigned on 3 July 1995.

\section{Professional staff appointments}

Masako Tsuruta, chief, Division of Clinical Laboratories, Department of Clinical Studies, Nagasaki, resigned as of 1 June 1995. Tomoko Nishikawa was employed as acting chief, Division of Clinical Laboratories, as of 2 June.

Akio Awa, associate chief of research, retired as of 30 June 1995.

Shizuyo Kusumi, assistant chief, Department of Clinical Studies, resigned as of 31 July 1995.

Masumi Abe, research scientist, Cell Biology Laboratory, Department of Radiobiology, Nagaksaki, resigned as of 20 February 1996.

Masanori Otake, assistant chief, Department of Statistics, resigned as of 31 March 1996.

\section{Mandatory retirement}

Fourteen employees in Hiroshima and 16 employees in Nagasaki reached the mandatory retirement age in FY95.

\section{Long Service Award ceremonies}

Long Service Award ceremonies were held on 4 April in Hiroshima and on 14 April in Nagasaki to express appreciation to employees (20 in Hiroshima and 3 in Nagasaki) for their many years of devoted service.

\section{Organizational structure of RERF}

\section{Department of Epidemiologic Pathology integrated into Department of Epidemiology}

With the change of data computing system from host computer system to workstation system and the completion of network computing system, the organizational structure of the Epidemiology Department was changed as of 1 July 1995, as follows:

The Departments of Epidemiologic Pathology in Hiroshima and Nagasaki were abolished, and the Tumor \& Tissue Registry Office and Laboratory of Pathology of those departments were integrated into the Department of Epidemiology, and the Field Investigation Section of the Department of Epidemiology was integrated into the Master File Section as the Investigation Unit. 


\section{Transfer of Library \& Archives to Research Information Center}

To enhance the convenience of providing information to research scientists through the medium of the library based on the concept of data processing and to improve library services by exchanging of scientific information with outside organizations through E-mail and Netscape, the organizational structures of the Research Information Center and Publication \& Documentation Center were partially changed, as of 1 July 1995, as follows:

Library \& Archives, Publication \& Documentation Center was renamed Library Section and transferred to Research Information Center.

Research Information Center was renamed Information Technology Department, and Information Systems Laboratory and Computer Section of that department were integrated into the Systems Technology Section.

Figures 1, 2 and 3, which follow on pages 119-121, show the present overall organizational structure and the structures of research departments and secretariat of the RERF.

\section{Fiscal and property report}

The RERF budget, settlement of accounts and property for FY95 are as shown in the tables below (Tables 3-6). The subsidy income, which is the main source of regular income, is equally provided by the governments of Japan and the United States based on the principle of equal support (Table 5).

The total RERF budget for operations was $¥ 4,730,982,000$, a decrease of $¥ 737,088,000(-13.48 \%)$ over the previous year. The major increase and decrease were a $¥ 795,534,000(-16.98 \%)$ decrease in personnel expenses and a $¥ 58,446,000$ $(+7.47 \%)$ increase in operational expenses (Table 3 ).

In the settlement of accounts for FY95, there was a surplus of $¥ 8,954,548$ in personnel funds, which was returned to the Japanese government (Table 5).

Table 3. Increase and decrease in operating costs (in yen)

\begin{tabular}{lccc}
\hline & \multicolumn{3}{c}{ Fiscal year } \\
\cline { 2 - 4 } Category & 1993 & 1994 & 1995 \\
\hline \multirow{2}{*}{ Personnel expenses } & $4,439,544,000$ & $4,685,974,000$ & $3,890,440,000$ \\
& $(102.8 \%)$ & $(105.6 \%)$ & $(83.02 \%)$ \\
Operational expenses & $845,764,000$ & $782,096,000$ & $840,542,000$ \\
& $(98.8 \%)$ & $(92.5 \%)$ & $(107.47 \%)$ \\
Total & $5,285,308,000$ & $5,468,070,000$ & $4,730,982,000$ \\
& $(102.2 \%)$ & $(103.5 \%)$ & $(86.52 \%)$ \\
\hline
\end{tabular}




\section{Figure 1. Organization of RERF}

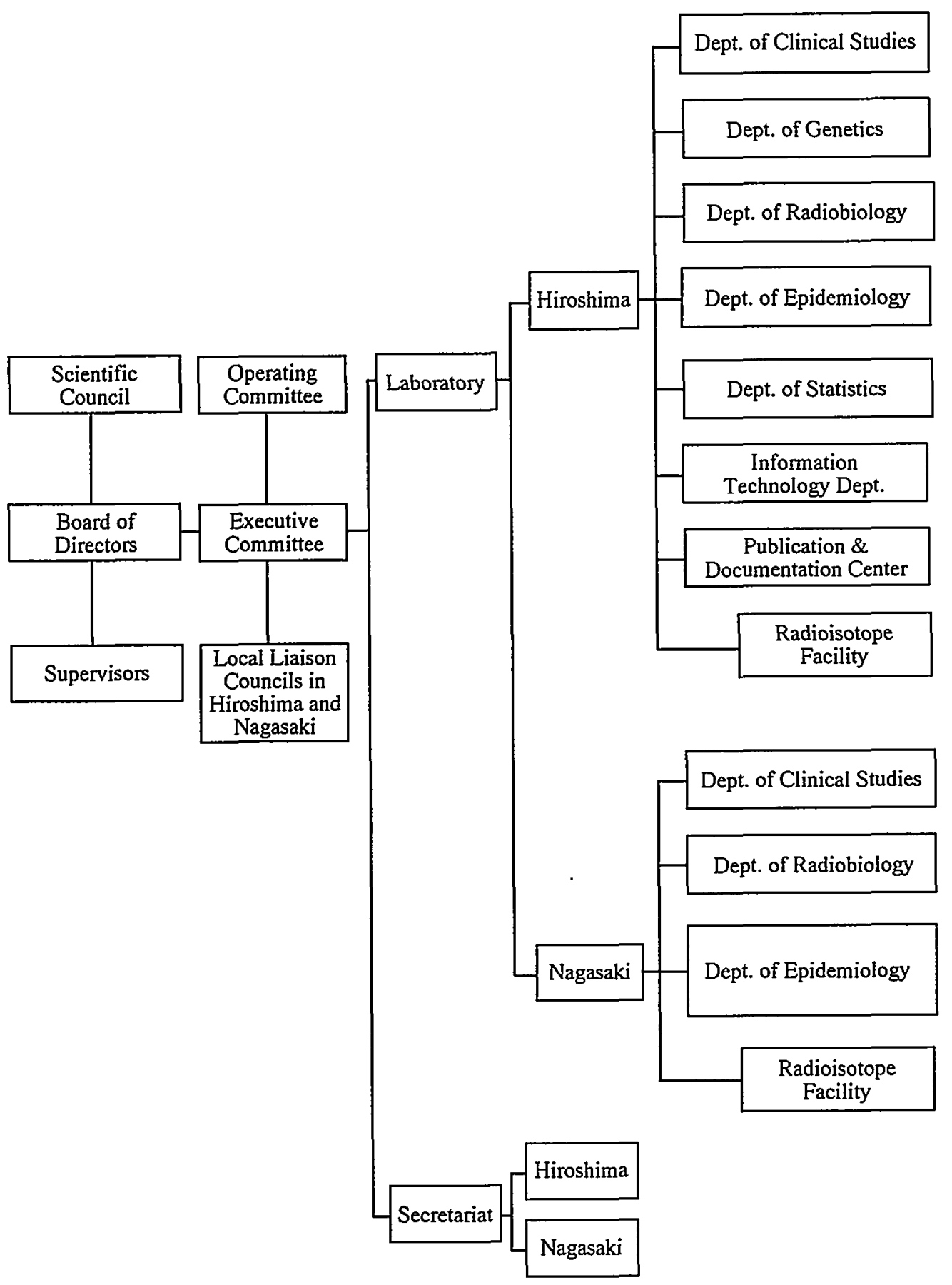




\section{Figure 2. Organization of the Laboratories}
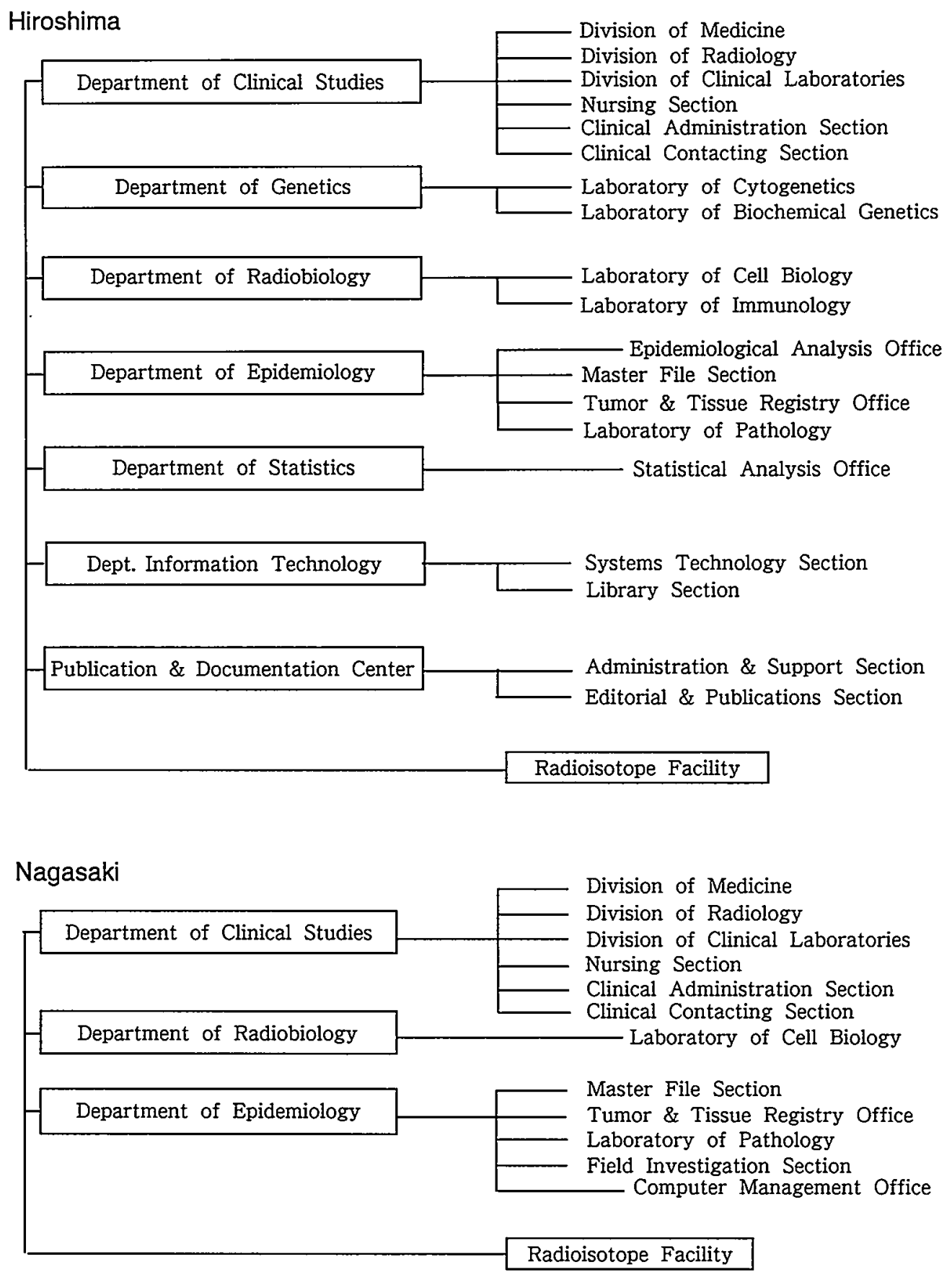
Figure 3. Organization of the Secretariat

Hiroshima

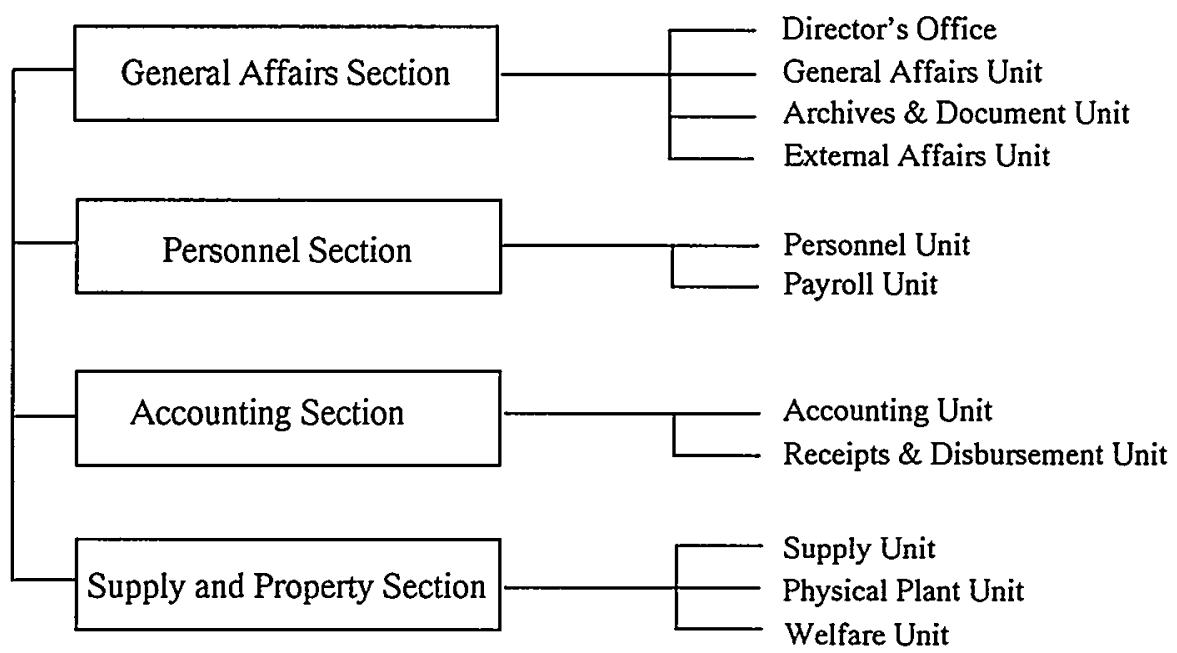

Nagasaki

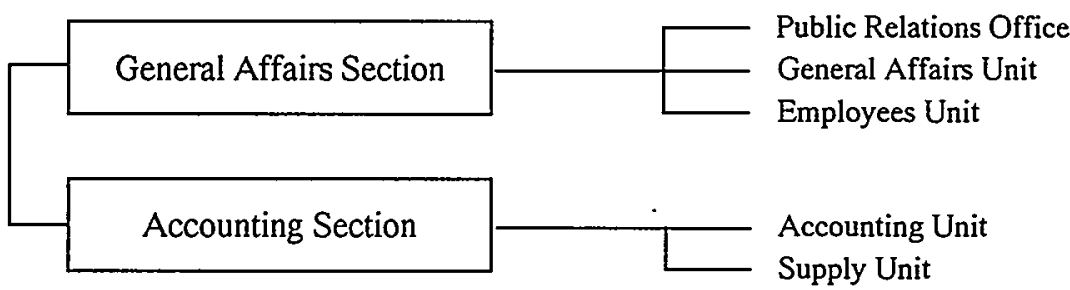


Table 4. Settlement of accounts, summary sheet, 1 April 1995-31 March 1996

Continued on right page

\begin{tabular}{|c|c|c|c|c|c|}
\hline Category & Total & $\begin{array}{l}\text { Regular } \\
\text { account }\end{array}$ & $\begin{array}{l}\text { Cancer } \\
\text { case-LSS } \\
\text { SP acct }\end{array}$ & $\begin{array}{l}\text { Radiation risk } \\
\text { assessment } \\
\text { SP acct }\end{array}$ & $\begin{array}{c}\text { Shigematsu } \\
\text { colla. study } \\
\text { SP acct }\end{array}$ \\
\hline \multicolumn{6}{|l|}{ Income } \\
\hline Subsidy & $4,693,733,452$ & $4,693,733,452$ & 0 & 0 & 0 \\
\hline $\begin{array}{l}\text { Operational } \\
\text { income } \\
\text { (interest, etc) }\end{array}$ & $66,993,346$ & $48,122,655$ & 9,015 & 0 & 1,583 \\
\hline $\begin{array}{l}\text { Transferred } \\
\text { from special } \\
\text { account }\end{array}$ & 0 & 0 & 0 & 0 & 0 \\
\hline $\begin{array}{l}\text { Entrustment } \\
\text { fund }\end{array}$ & $82,032,069$ & 0 & $15,171,185$ & $2,987,000$ & $16,700,000$ \\
\hline $\begin{array}{l}\text { Carried over } \\
\text { from previous } \\
\text { year }\end{array}$ & $852,872,371$ & 0 & $20,530,240$ & 0 & 0 \\
\hline Total income & $5,695,631,238$ & $4,741,856,107$ & $35,710,440$ & $2,987,000$ & $16,701,583$ \\
\hline \multicolumn{6}{|l|}{ Expenditures } \\
\hline $\begin{array}{l}\text { Personnel } \\
\text { expenses }\end{array}$ & $4,207,910,432$ & $3,881,352,904$ & $5,849,256$ & 0 & 0 \\
\hline $\begin{array}{l}\text { Operational } \\
\text { expenses }\end{array}$ & $930,911,551$ & $851,548,655$ & $29,861,184$ & $2,987,000$ & $16,701,583$ \\
\hline Total expenses & $5,138,821,983$ & $4,732,901,559$ & $35,710,440$ & $2,987,000$ & $16,701,583$ \\
\hline $\begin{array}{l}\text { Balance, income } \\
\text { minus expenses }\end{array}$ & $556,809,255$ & $8,954,548$ & 0 & 0 & 0 \\
\hline
\end{tabular}

Note: LSS = Life Span Study; SP acct = special account; colla. = collaborative; NAS = National 
Continued from left page

\begin{tabular}{|c|c|c|c|c|c|}
\hline $\begin{array}{l}\text { Termination } \\
\text { trust fund } \\
\text { SP acct }\end{array}$ & $\begin{array}{l}\text { NAS } \\
\text { housing } \\
\text { SP acct }\end{array}$ & $\begin{array}{l}\text { Nagasaki Pref. } \\
\text { Cancer Registry } \\
\text { SP acct }\end{array}$ & $\begin{array}{c}\text { A-bomb } \\
\text { memorial } \\
\text { SP acct }\end{array}$ & $\begin{array}{l}\text { Nonsubsidy } \\
\text { expenses } \\
\text { SP acct }\end{array}$ & $\begin{array}{c}\text { Epi. study Mayak } \\
\text { \& Techa river } \\
\text { SP acct }\end{array}$ \\
\hline \multirow[b]{2}{*}{$16,730,801$} & 0 & 0 & 0 & 0 & 0 \\
\hline & 565,455 & 0 & 0 & $1,563,837$ & 0 \\
\hline 0 & 0 & 0 & 0 & 0 & 0 \\
\hline 0 & $1,699,835$ & $7,500,000$ & $34,469,000$ & 0 & $3,505,049$ \\
\hline $831,081,362$ & $1,260,769$ & 0 & 0 & 0 & 0 \\
\hline $847,812,163$ & $3,526,059$ & $7,500,000$ & $34,469,000$ & $1,563,837$ & $3,505,049$ \\
\hline $304,824,732$ & 0 & $5,988,699$ & $9,894,841$ & 0 & 0 \\
\hline 0 & $2,163,832$ & $1,511,301$ & $24,574,159$ & $1,563,837$ & 0 \\
\hline $304,824,732$ & $2,163,832$ & $7,500,000$ & $34,469,000$ & $1,563,837$ & 0 \\
\hline $542,987,431$ & $1,362,227$ & 0 & 0 & 0 & $3,505,049$ \\
\hline
\end{tabular}

Academy of Sciences; Pref. = Prefecture; Epi. = Epidemiologic; all amounts are in yen . 
Table 5. Statement of income and expenditures, regular account, 1 April 1995-31 March 1996

\begin{tabular}{|c|c|c|c|}
\hline Category & $\begin{array}{c}\text { Budget } \\
\text { A }\end{array}$ & $\begin{array}{c}\text { Settlement } \\
\text { B }\end{array}$ & $\begin{array}{c}\text { Balance } \\
\mathrm{A}-\mathrm{B}\end{array}$ \\
\hline \multicolumn{4}{|l|}{ Income } \\
\hline Subsidy income & $¥ 4,702,688,000$ & $¥ 4,693,733,452$ & $¥ 8,954,548$ \\
\hline GOJ treasury & $2,351,344,000$ & $2,351,344,000$ & 0 \\
\hline GUS treasury & $2,351,344,000$ & $2,342,389,452$ & $8,954,548$ \\
\hline Independent income & $28,294,000$ & $48,122,655$ & $\triangle 19,828,655$ \\
\hline Medical exams (ABSMTL) & $28,294,000$ & $24,817,220$ & $3,476,780$ \\
\hline Miscellaneous & 0 & $23,305,435$ & $\triangle 23,305,435$ \\
\hline Total income (A) & $4,730,982,000$ & $4,741,856,107$ & $\Delta 10,874,107$ \\
\hline \multicolumn{4}{|l|}{ Expenditures } \\
\hline Personnel expenses & $3,890,440,000$ & $3,881,352,904$ & $9,087,096$ \\
\hline Salary & $2,957,718,000$ & $2,934,250,556$ & $23,467,444$ \\
\hline Termination allowance & $932,722,000$ & $947,102,348$ & $\triangle 14,380,348$ \\
\hline Operational expenses & $840,542,000$ & $851,548,655$ & $\triangle 11,006,655$ \\
\hline Fees \& gratuities & $24,988,000$ & $25,567,213$ & $\triangle 579,213$ \\
\hline Travel, directors \& staff & $15,663,000$ & $15,771,535$ & $\triangle 108,535$ \\
\hline Travel, committee & $5,584,000$ & $5,485,472$ & 98,528 \\
\hline Travel, relocation & $5,256,000$ & $4,958,398$ & 297,602 \\
\hline Travel, overseas & $4,000,000$ & $4,185,781$ & $\Delta 185,781$ \\
\hline Office \& lab. expenses & $714,441,000$ & $726,132,898$ & $\Delta 11,691,898$ \\
\hline Rent, land \& buildings & $52,846,000$ & $52,843,388$ & 2,612 \\
\hline Maintenance \& repair & $16,864,000$ & $15,779,250$ & $1,084,750$ \\
\hline Taxes & 900,000 & 824,720 & 75,280 \\
\hline Total expenditures (B) & $4,730,982,000$ & $4,732,901,559$ & $1,919,559$ \\
\hline
\end{tabular}

Balance (A) - (B)

(FY95 personnel $\quad 0 \quad 8,954,548$

expenditures surplus)

Note: GOJ = Government of Japan; GUS = Government of the US; ABSMTL = Atomic Bomb Survivors' Medical Treatment Law. RERF receives a fee for every medical examination of an atomic-bomb survivor, as specified in the national law.

$\Delta$ Indicates income or expenditures in excess of budget 
Table 6. Regular account balance sheet as of 31 March 1996

\begin{tabular}{|c|c|c|}
\hline & Category & Amount \\
\hline \multicolumn{3}{|l|}{ Assets } \\
\hline \multirow[t]{6}{*}{ Current assets: } & Cash, deposits & $¥ 348,689,572$ \\
\hline & Accounts receivable & $4,061,687$ \\
\hline & Accounts prepaid & $3,533,689$ \\
\hline & Interfund receivable & $5,376,084$ \\
\hline & Prepayment & $1,149,940$ \\
\hline & Total current assets & $362,810,972$ \\
\hline \multirow[t]{3}{*}{ Fixed assets: } & Buildings & $226,372,644$ \\
\hline & Equipment & $438,575,885$ \\
\hline & Total fixed assets & $664,948,529$ \\
\hline Total assets: & & $\mathbf{1 , 0 2 7 , 7 5 9 , 5 0 1}$ \\
\hline \multicolumn{3}{|l|}{ Liabilities } \\
\hline \multirow[t]{5}{*}{ Current liabilities: } & Accounts payable (regular account) & $25,160,419$ \\
\hline & Salary deductions on hand & $19,254,319$ \\
\hline & $\begin{array}{l}\text { Balance of subsidy to be returned to the } \\
\text { Japanese government }\end{array}$ & $8,954,548$ \\
\hline & $\begin{array}{l}\text { Balance of subsidy to be returned to the } \\
\text { US government }\end{array}$ & 0 \\
\hline & Total current liabilities & $53,369,286$ \\
\hline \multirow[t]{2}{*}{ Fixed liabilities: } & Termination allowance reserve fund & $309,441,686$ \\
\hline & Total fixed liabilities & $309,441,686$ \\
\hline Total liabilities: & & $362,810,972$ \\
\hline \multirow[t]{4}{*}{ Property funds } & Basic property (buildings) & $226,372,644$ \\
\hline & Operating property (equipment) & $438,575,885$ \\
\hline & Total property funds & $664,948,529$ \\
\hline & Total liabilities and property funds & $1,027,759,501$ \\
\hline
\end{tabular}




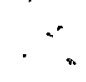




\section{Appendix}


$\therefore$ 


\section{9th Board of Directors Meeting}

\section{6-28 June 1995}

\section{Auditorium, Radiation Effects Research Foundation Hiroshima}

\section{Agenda}

I. Minutes of the 28th meeting of the Board of Directors

II. Items for information

1. Status report of RERF

2. Present personnel status

3. Fiscal Year 1994 (FY94) salary revision, etc.

4. Labor Union's Fiscal Year 1995 (FY95) demands for improvement of working conditions, etc.

5. Others

(1) International collaboration

(2) Development of an A-bomb related materials database system

(3) Dosimetry reassessment

III. Items for deliberation and action

1. Recommendations of the 22nd meeting of the Scientific Council

2. FY94 research activities report and audit report

3. FY95 research plans

4. FY94 settlement of accounts and audit report

5. FY95 working budget

6. Outline of FY96 budget request

7. Revision of rules and regulations

8. Election/appointment of directors and others

9. Schedule of next board meeting

\section{Participants}

\section{Full-time officers}

Itsuzo Shigematsu, chairman

Mortimer L Mendelsohn, vice chairman

Donald R Harkness, permanent director and chief of research

Yutaka Hasegawa, permanent director 


\section{Visiting directors}

Kazuaki Arichi, director, Japan Institute of International Affairs

Toshiyuki Kumatori, chairman, Radiation Effects Association

Seymour Jablon, expert, Radiation Epidemiology Branch, Division of Cancer Etiology, National Cancer Institute

Warren K Sinclair, president emeritus, National Council on Radiation Protection and Measurements

Joseph E Rall, deputy director for international research, US National Institutes of Health

\section{Supervisors}

Akira Shishido, former director, Japanese National Institute of Health

David Williams, senior financial advisor, US National Academy of Sciences (NAS)

\section{Co-chairman of the RERF Scientific Council}

Ei Matsunaga, professor emeritus, Japanese National Institute of Genetics

\section{Observers}

Hiroshi Maruyama, deputy director, Planning Division, Health Service Bureau, Ministry of Health and Welfare (MHW)

Tadashi Fujitani, chief, Medical Care Activities Unit, Planning Division, Health Service Bureau, MHW

Paul Seligman, deputy assistant secretary for health, US Department of Energy (DOE)

Milton Eaton, DOE Representative, US Embassy, Tokyo

John D Zimbrick, director, Board on Radiation Effects Research, Commission on Life Sciences, National Research Council, NAS

Catherine S Berkley, administrative associate, Commission on Life Sciences, National Research Council, NAS

\section{Operating Committee members of RERF}

Yasukiyo Hirano, chief of secretariat

Akio Awa, associate chief of research

Jill L Ohara, chief of Research Information Center

Richard D Sperry, advisor, Secretariat

Tadashi Nakaoka, chief of administration, Secretariat, Nagasaki Laboratory 


\section{Minutes of the meeting}

The 29th meeting of the board of directors of the Radiation Effects Research Foundation was held 26-28 June 1995, in the auditorium of RERF's Hiroshima Laboratory.

Nine directors except for Dr Saburi were present. This satisfied the quorum required under the Act of Endowment and formally constituted the board meeting. Dr Shishido and Mr Williams, the RERF supervisors, Dr Matsunaga, co-chairman of the RERF scientific council were also present. The observers in attendance included representatives from the Japanese Ministry of Health and Welfare (MHW), the US Department of Energy (DOE), as well as representatives of the US Embassy and the National Academy of Sciences (NAS).

The board appointed Dr Harkness and Dr Hasegawa as signatories of the minutes of this meeting. Dr Shigematsu, chairman of RERF, presided over the meeting as provided in the Act of Endowment.

I. Minutes of the 28th meeting of the Board of Directors (presented by Itsuzo Shigematsu)

With respect to the issue of making a thorough review of the RERF budget and personnel, which is now in progress as a two-year plan starting on 1 April 1994, Dr Shigematsu requested that the board deliberate, as part of the "status report of RERF" to be discussed later, on the question of how to maintain the present level of research in the face of the decreasing number of employees year after year and offer suggestions. The board members agreed to deliberate on the issue later and approved the minutes.

\section{Items for information}

Dr Shigematsu suggested that a progress report on dosimetry assessment be made as an additional item for information, including the points discussed at the dosimetry committee meeting held prior to the board meeting. For this purpose, he volunteered to report the activities of the Japanese group and asked Dr Sinclair to cover the US activities.

\section{Status report on RERF (presented by Mortimer $L$ Mendelsohn and Itsuzo Shigematsu)}

The board members agreed to deliberate, at the session for deliberation, on the issue of cost reduction due to the accelerating financial difficulties of the US side since the year before last, and plans for personnel reduction to cope with the budgetary reduction. A report was made of the situation which developed after last year's board meeting, including the transfer of the contract DOE has with NAS.

While the cost reduction plan starting from the fiscal year 1994 was being carried out, DOE served notice in January this year to effect a plan to terminate 
the contract it has with NAS for the management of RERF in March and change its contractor to an American university. However, the scientific council recommended at its meeting in April that a blue ribbon committee be established to discuss RERF's future and that DOE defer the proposed termination of its contract with NAS until the blue ribbon committee makes its recommendations. Later, NAS adopted a similar resolution at its annual meeting. With this background, RERF proposed to DOE that it abandon its intention to terminate its contract with NAS and hold a binational meeting to discuss these issues.

An agreement was reached between DOE and MHW in May on the RERF budget for this fiscal year (FY95).

In June, DOE Secretary O'Leary wrote a letter to NAS President Alberts saying that "it is now time to bring the foundation into the mainstream of scientific and public health research" and that "the need for secret research on the biological effects of radiation has ended." Dr Shigematsu requested that the contents of this letter be responded calmly because RERF had sent a letter of protest to DOE.

The US side is somewhat disappointed because personnel and organizational streamlining at RERF is not proceeding as rapidly as it should while attrition of the study population is accelerating.

No doubt the financial problems of RERF have their source in the budgetary constraint practiced in the US. On the other hand, Japan's position seems to be that the US-Japan equal-sharing system has its source in the Notes Verbales exchanged between the Japanese and US governments and cannot be changed easily. Another point is that, while the US is in favor of collaboration of Japan and the US in the core projects of RERF, such as mortality studies and cancer studies, it wants to pull out from other projects.

As to the RERF relocation issue, a report was made to the effect that, although consideration of relocation had been suspended in FY93 in deference to the request of DOE, MHW has not removed the question from its agenda for discussion.

Lastly, a report was made that the A-bomb Survivors Relief Law will take effect on 1 July 1995 and that the operational basis of RERF has been established legally and financially in the law for the first time and this has farreaching implications.

\section{Present personnel status (presented by Yasukiyo Hirano)}

The personnel strength as of 1 April 1995 was 4 directors, 1 chief of secretariat, 48 research scientists, and 320 general employees, totaling 373, which represents a decrease by 39 employees since last year. The breakdown of the 320 general employees is: 216 in administrative positions, 18 in manual and general technical positions, 66 in medical and technical (I) positions, and 20 in 
medical and technical (II) positions. Although the number of employees in administrative positions seems large, 158 of them are engaged in research supporting activities such as tumor and tissue registries, computing, and medical recording. The remaining 58 are in the secretariat.

As for the changes in the actual personnel strength, it was 588 in 1975, when RERF was established, and now, after 20 years, it has dropped to 373 , which is $63 \%$ of that in 1975 . In particular, the personnel reduction caused by the budget reduction in FY93 and FY94 has led to the present situation. Furthermore, although 94 employees are due for retirement within five years from this fiscal year, the policy is that, in principle, no replacements will be employed, be the retirement mandatory or voluntary, unless such positions are deemed indispensable. Therefore, no personnel increase can be expected in the foreseeable future.

\section{FY94 salary revision, etc (presented by Yasukiyo Hirano)}

The pay scales were revised by using as a guide the revision of the pay scales applicable to national government employees. The base pay for professional employees (average age 44.01) and that for general employees (43.11) were increased by $1.12 \%$ and $1.16 \%$ on average, respectively, whereas the increase for national government employees (39.5) was $1.18 \%$. Dependents' allowance was increased, but the yearly rate of seasonal allowances continued to be decreased for two consecutive years from last year to 5.2 months' pay (last year 5.3 months).

Revision of the pay scale applicable to the directors, which is decided by using the pay scale applicable to national government employees in designated positions, due in FY94 was suspended. It is expected to be effected as of 1 April 1995.

\section{Labor Union's FY95 demands for improvement of working conditions,} etc (presented by Yasukiyo Hirano)

The membership of the RERF Labor Union numbers 223, which amounts to $60 \%$ of all the directors and staff combined. If persons ineligible for Union membership such as directors, research scientists and those in managerial positions are excluded, it represents $92 \%$. The RERF Labor Union is affiliated with All Japan Prefectural and Municipal Workers' Union, and is backed up by the latter's Hiroshima prefectural headquarters in the same way it is by other supporting higher organizations.

Taking note of the fact that the legal and financial status of RERF has been established following enactment of the A-bomb Survivors Relief Law, the FY95 demands contain the following four points: 1) revision of the US-Japan equal sharing system; 2) representation of A-bomb survivors on the RERF board of directors; 3) establishment of a voting organ (council) for monitoring actions of 
the RERF Board of Directors; and 4) relocation of RERF and alongside it, reopening of the department that provides treatment.

In addition, demands for improvement of working conditions such as salary increases and participation in the welfare pension fund are included.

\section{Others}

(1) International collaboration (presented by Itsuzo Shigematsu)

The majority of international collaborative activities involving RERF staff were Chernobyl-related. There are six channels including the World Health Organization through which RERF participates in international collaborative activities. RERF also twice took part in activities related to Chelyabinsk, for which it has a collaborative agreement.

Last year RERF received 285 official visitors. Although their visits did not concentrate on particular departments, the question of how visitors, whose stay at RERF varies from a few days to a few months, should be accommodated without disrupting regular activities remains to be an important subject for further discussion.

RERF has been designated as a research collaborative center of WHO. However, from the aforementioned international collaborative activities, it is clear that the activities of RERF in reality exceeds the level of what is expected of a designated research collaborative institute of WHO. Therefore, it was recommended and resolved at the board meeting two years ago that RERF be made a research center directly attached to the United Nations.

(2) Development of an A-bomb related materials database system (presented by Yutaka Hasegawa)

The objective of this project is to develop and investigate a database system to be installed in the "A-bomb Victims Memorial Hall", which MHW is planning to build. The project is being carried out under a contract with MHW.It was reported that progress had been made in the following four areas last year. 1) preparation of a list of locations of documents relating to measures that have been taken for care of A-bomb survivors; 2) inputting data from the surveys of MHW on the actual conditions of A-bomb survivors to the optical disk filing system; 3 ) preparation of explanatory notes on major A-bomb-related items and terms and development of a system to display them on the computer screen; and 4) investigations of overseas organizations that may possess A-bomb-related materials.

(3) Dosimetry reassessment (presented by Warren $K$ Sinclair and Itsuzo Shigematsu)

Dr Sinclair first reported the history of development of the dosimetry system including the activities of the US dosimetry committee (chairman: Warren K Sinclair). Subsequently, Dr Shigematsu explained the status of the 
activities of the Japanese committee on behalf of Dr Eizo Tajima, chairman of the committee. The Japanese committee is continuing to collect exposed materials in Hiroshima and Nagasaki and to measure residual radioactivity, and plans to begin this year the estimation of radiation dose by the ESR measurement of tooth enamel.

\section{Items for deliberation and action}

\section{Recommendations of the 22nd meeting of the Scientific Council (presented by Ei Matsunaga)}

The Scientific Council, which met in April this year, not only evaluated the direction and adequacy of the research projects but also made recommendations on the issues concerning the mandate for RERF's budget reduction, which has its source in the aggravation of the financial situation in the US, and the subsequent personnel reduction problem. Also, it made recommendations concerning the issue of termination of the contract DOE has with NAS for the management of RERF, which surfaced in January this year, and suggested that opinions from a wide viewpoint be sought on the future direction of RERF.

With regard to specific research studies, it was recommended that the epidemiological and statistical studies, which are regarded by the councillors as one of the core programs of RERF, be continued without disruption. Further, the council proposed that low-dose radiation exposure and mortality from noncancer diseases which are statistically shown to be related to radiation dose be studied, and that A-bomb survivors who were exposed at younger ages and are reaching cancer age be followed up closely.

It was also recommended that the AHS be continued. Good results have been obtained from a preliminary mail survey followed by telephone contacts, which were made at intervals in between biennial health examinations in view of the aging and attrition of the AHS population. The council recommended continuation of this method. High radiation risks have been shown for the breast and colon cancers which developed at younger ages in the AHS population. Thus, studies on genetic susceptibility should be given top priority.

The Research Information Center took the central role in the changeover of the host computer system to the work station system and personal computers and completed it about six months earlier than originally scheduled. The new system is considered to be contributing to the research projects greatly.

The Department of Genetics is making progress in developing a twodimensional electrophoretic system for detecting possible germ-line mutations in children of exposed and nonexposed A-bomb survivors. This study is being conducted in collaboration with the University of Michigan which is a good example of successful joint studies of RERF with a university. A great interest 
was taken in the ESR measurements of tooth enamel, which is a new method of dosimetry, and its continuation was recommended.

The main purpose of the molecular epidemiology studies in the Department of Radiobiology is to determine whether damages unique to ionizing radiation exposure are observed in cancer tissues. Comparison with data obtained elsewhere is required to support the findings. Other activities include studies of mutations occurring in somatic cells; and remarkable progress was reported in studies of the somatic gene mutations of glycophorin A and of T-cell receptors. New models have been developed for studying radiation effects in human cells and tissues transplanted into SCID mice. Interestingly the dose response for epilation of human hair using this model is very similar to that which occurred in the exposed survivors. The Council evaluated this as an excellent study in good accord with the mission of RERF. With repeated reference to the high quality of the RERF studies, explanation of the individual studies was concluded.

Discussion then moved on to the Scientific Council's general recommendations. The Scientific Council, held this year amid great turmoil caused by the financial instability at RERF continuing from the year before last and DOE's proposal in January to terminate its contract with NAS, requested Chief Medical Officer Galson of DOE and Assistant Director Maruyama of MHW to explain the current situation and each of the department chiefs present to state his/her opinion. The Scientific Councilors, taking the position that they are charged to ensure that the scientific activities of RERF are carried out properly and smoothly while maintaining the highest scientific standards, have concluded that it is important to seek, on the occasion of the 50th anniversary of the atomic bombing, the recommendations of an international (blue ribbon) committee concerning RERF's future scientific activities. To enable RERF to fulfill its international mission beyond the existing binational arrangement, this committee should consist of distinguished scientists who have had little association with RERF in the past and should carry out an assessment of RERF's current and future activities from a broad perspective with support from both the US and Japan. The Scientific Council supported the idea of strengthening the training of radiation biologists and scientific exchange activities, which has its source in DOE's proposal to terminate the contract it has with NAS, considering the favorable effect it would have on RERF.

Considering the possibility of greater budgetary constraints, RERF scientists were encouraged to apply for research grants from various organizations both in Japan and abroad and also make whatever efforts they can in the interest of maintaining a favorable research environment.

To the US and Japanese governments, the Council requested that RERF be operated with due consideration of its unique binational character. 
Lastly, considering the course of events so far, the Council recommended that DOE defer its current plan of discontinuing NAS sponsorship until such time that the blue ribbon committee proposed by the Council makes its report on the future of RERF.

Dr Sinclair asked how genes were collected from young A-bomb survivors to study breast cancer among them. Dr Matsunaga and other directors reported that a study on "BRCAl" and other genes using tissue samples was in the planning stage and that epidemiological data on breast cancer indicated the risk to be 10 -fold higher in young survivors. It was reported that, considering the limited samples available at RERF, access to resources elsewhere was a matter for consideration in the future.

Preliminary results of the dosimetry study being conducted using tooth enamel showed good correlation with DS86 and was considered to be an important undertaking which may lead to improvement in dose assessment.

As for the general recommendations, discussion focused on the recommended blue ribbon committee, from the viewpoint that it may lead to the solution of the problem arising from DOE's proposed termination of the contract with NAS, and the representatives of DOE and MHW attending the meeting as observers were asked for their opinions. Dr Rall made a motion to approve the recommendations of the Scientific Council. The motion was seconded and the recommendations were approved.

\section{FY94 research activities report and audit report (presented by Donald R Harkness)}

Dr Shigematsu first suggested that the FY94 research activities report and the FY95 research plans be presented and deliberated on together because these two were closely connected. This suggestion was agreed on.

Dr Harkness gave a general explanation on the research activities, prefacing that all was more specifically described in the report from each department attached as material prepared for the board meeting.

Dr Harkness reported that there were, as discussed a number of times, many problems hindering the conduct of research activities; namely, 1) the uncertainty due to the issue concerning DOE's contract with NAS, 2) the visit to the foundation of numerous people including individuals from the mass communication media, not to mention researchers, especially because this year marked the 50th anniversary of the bombings, 3) the transition from a mainframe computer to workstations, and 4) the restriction on research expenditures because the FY94 working budget had not been established until last October due to the financial situation of the US side.

Under these circumstances, only two RPs have been approved this year, one is a pilot study and the other essentially an addendum to an earlier study. The 
number of planned applications for review of possible new RPs has decreased substantially. It was difficult to submit new RPs in the uncertain budgetary situation; however, he stated that the quality of the research studies conducted in FY94 was very good, considering the problems mentioned above.

Then Dr Shishido presented his supervisor's report on scientific activities, deeming "the contents of the scientific activities report for FY1994 to be true and correct," and commented that he is worried over the future of RERF.

\section{FY95 research plans (presented by Donald R Harkness)}

Dr Harkness gave a general description of the FY95 research plans without going into detail and then commented on possible courses of action for RERF.

Long-term countermeasures are needed to address the required personnel decreases and uncertain research budgets caused by today's unstable financial basis arising from the exchange rate and other problems. The feasible measure that RERF can resort to is prioritization of the research projects. Prioritization is difficult for parties directly involved, so the research projects should be reviewed and their future direction set by a third party such as a blue ribbon committee.

In response to this report, an opinion was presented that it would be more preferable to have RERF directors, rather than those who are not familiar with RERF, make a review and set the future direction, receiving the advice of scientific councillors. However, because the board had adopted the recommendations of the RERF Scientific Council which include establishment of a blue ribbon committee on the first day of its meeting and this issue was one of the most important topics of this board meeting, the board agreed on establishment of a blue ribbon committee and approved the FY94 research activities report and FY95 research plans.

\section{FY94 settlement of accounts and audit report (presented by Yasukiyo Hirano)}

Details of the FY94 regular account and special accounts were explained.

The FY94 budget originally approved by MHW had been $¥ 5,460,000,000$ $(¥ 2,730,000,000$ each to be borne by Japan and the US). The final budget adopted, following a series of US-Japan negotiations upon receipt of information that the $\$ 18,000,000$ would be the maximum the US could provide, was $¥ 4,344,074,000$ ( $¥ 2,153,479,000$ each to be borne by Japan and the US). To adjust to this austerity budget, the following plans were developed and implemented:

1) Minimum employment of replacements-Of the 40 retirements and resignations, only six replacements were employed.

2) Elimination of two positions for permanent directors-Effected 1 July 1994. 
3) Exclusive appointment of chief of secretariat (release of permanent director from concurrent appointment)-Effected 1 July 1994.

4) Reduction of researchers dispatched from universities-Reduction of three by 31 March 1995 and a fourth to occur in July or August 1995.

5) Reorganization of the secretariat including elimination of positions for administrators, reduction in the number of assistant chiefs of secretariat and reduction of sections from six to four-Effected 1 July 1994.

6) One-year suspension of the revision of pay scale for directors-Effected 1 April 1995.

As a result of the above actions and others, the income in the regular account reported in the settlement of accounts was $¥ 4,435,629,367$, of which $¥ 4,381,317,000$ was the total of the subsidies from Japan and the US, $¥ 54,295,303$ independent income from the activities of the foundation and $¥ 17,064$ the balance in the special account of Hijiyama Hall dining room which was closed in June 1994. The reported expenditures were $¥ 4,358,943,297$, of which $¥ 3,559,650,930$ was for personnel expenses and $¥ 799,292,367$ for operating expenses. Of the balance of $¥ 76,686,070$ of income, arrangement has been made to return $¥ 75,522,535$ and $¥ 1,163,535$ to the treasuries of Japan and the US, respectively.

Mr Williams, supervisor, gave a report of his audit as of 31 March 1995. A decrease of $1.7 \%$ in yen terms in the unfunded termination liability was welcomed, but it was pointed out that there was an increase of $15 \%$ in dollar terms. There has been a further decrease in the value of the dollar against the yen currency as observed in the yen-dollar exchange rate since the audit.

Completion of the A-133 audit (audit required by the US Office of Management and Budget) and the A-122 audit (audit to comply with US government regulations) was reported. There were no irregularities.

In response to the question regarding the practice of the Board of Audit of the Japanese government, the chief of secretariat reported that there had been an audit twice in the past, 1984 and 1985, and that appropriate actions had been taken each time. As to whether there were legal problems which discourage the foundation from accumulating a portion of its income to meet the growing termination liability as a greater number of employees reach retirement age, the chief of secretariat said that at present this was not necessary because Japan and the US had an understanding that funds for termination payouts will be provided as needed in the yearly budgets.

With this, the 1994 settlement of accounts was approved.

\section{FY95 working budget (presented by Yasukiyo Hirano)}

A budget of $¥ 4,501,410,000$ had been decided for FY95 at the US-Japan talks in November 1994, but the subsequent drastic change in the yen-dollar 
exchange rate made execution impossible for the US side and reduction of the budget was requested by both governments. A solution by a plan incorporating the following was considered: 1) Each department will promote further rationalization, hiring no replacements for mandatory age retirements and voluntary retirements and covering the vacancies by transfers, and 2) A plan for reduction of the working budget was adopted, which included the following measures:

i) Deferment of introduction of the welfare pension fund to 1996 or later.

ii) Reduction of overtime allowance.

iii) Setting the rate of salary increase for FY95 at $1 \%$ or less (rate by the original plan: $2 \%$ ).

iv) $20 \%$ reduction of the operational expenses, which had been approximately $¥ 790,000,000$ in the original budget.

Reduction by the above plan totaled $¥ 273,570,000$. Deduction of this from the total amount agreed upon by US and Japan made the total amount of the budget $¥ 4,227,840,000$. This amount, which RERF found to be the absolute minimum with which it can operate, was approved by both governments in May this year.

In the total budget of $¥ 4,227,840,000$, personnel expenses are slightly increased over the previous year because of the increase for termination allowances, despite a reduction of $¥ 211,780,000$ for salaries. The operating expenses are decreased compared with the previous year, where possible $20 \%$ reductions were made for each category. However, since some categories cannot be decreased (eg, rent, insurance, utilities, etc), others were decreased more (eg, foreign travel and equipment were reduced to only $33 \%$ ).

This plan, providing for the minimum required for the operation of RERF, was approved.

\section{Outline of FY96 budget request (presented by Yasukiyo Hirano)}

It was explained that the substantial reduction made in the budget for FY95 would place the operations of the foundation in a critical state, and that although the budgets of the Japanese and US governments were as yet undecided, it was desired to make the budget request for FY 96 so that the budget can be restored to its former level.

The number of personnel, 335 at the beginning of the fiscal year, will become 312 at the end of the fiscal year with 23 expected to retire. Due to this, personnel expenses amounting to $¥ 3,203,520,000$, or $89.17 \%$ of the amount of the previous year, will be requested. As operational expenses, $¥ 805,980,000$, or $126.88 \%$ of the amount of the previous year (a slight increase over restoration of the $20 \%$ reduction for FY95), will be requested in accord with the aim of the 
budget request. Further, $¥ 31,910,000$ will be requested for joining the welfare pension fund, which was deferred in FY95.

Various views were presented concerning the fact that allocation of the working budget by project was the same for the past three years. A number of problems were pointed out, such as that the projects were assigned no comparative merits, which is necessary especially in a situation where the budget is being reduced. The Secretariat's explanation was acknowledged that the allocations were calculated based on the figures of the previous year and that there was no problem in the execution.

Although the budgets of the Japanese and US governments were still undecided, the budget request was approved by the Board in that the rationalization plan was being implemented.

\section{Revision of rules and regulations (presented by Yasukiyo Hirano)}

Revision of rules and regulations was proposed as follows, and each of the proposed revision was approved.

(1) Regulations Concerning Organization of the Laboratory (Research Departments)

Following reevaluation of work necessitated by the changeover of the ACOS host-computer system to the workstation system and the establishment of the network processing system, the Laboratory will be reorganized as follows:

\section{[Hiroshima]}

1) The Department of Epidemiologic Pathology will be incorporated into the Department of Epidemiology.

2) The name of the Master File Section of the Department of Epidemiology will be changed (Japanese only).

3) The Investigation Unit of the Field Investigation Section, Department of Epidemiology, will be transferred to the Master File Section, and the Data Collection Unit will be transferred to the Tumor \& Tissue Registry Office, Department of Epidemiology. As a result, the Field Investigation Section will be abolished.

4) A Registry Unit will be established in the Tumor \& Tissue Registry Office.

[Nagasaki]

1) The Department of.Epidemiology \& Biometrics and the Department of Epidemiologic Pathology will be combined and called the Department of Epidemiology.

2) The name of the Master File Section of the Department of Epidemiology will be changed (Japanese only), and the Records \& Archives Unit in that section will be abolished. 
3) The Investigation Unit of the Field Investigation Section, Department of Epidemiology, will be transferred to the Master File Section, and the Data Collection Unit will be transferred to the Tumor \& Tissue Registry Office, Department of Epidemiology. As a result, the Field Investigation Section will be abolished.

4) A Registry Unit will be established in the Tumor \& Tissue Registry Office.

(Effective date: 1 July 1995.)

(2) Regulations Governing Salaries of Directors and Chief of Secretariat

1) The base pay of the directors and chief of secretariat was increased by $1.18 \%$ on average by using as a guide the revision of the National Government Payscale for Designated Positions.

2) The yearly rate of seasonal allowances was decreased by 0.1 month's pay to 4 months' (currently 4.1 months' pay).

(Effective date: 1 April 1994. However, the action prescribed in paragraph 1) was delayed and became effective on 1 April 1995.)

(3) Employee Wage Regulations

1) The base pay of the employees was increased (for general employees by $1.16 \%$ and for research scientists by $1.12 \%$ ) by using as a guide the revision of the National Government Payscale for Designated positions.

2) The dependents' allowance was increased.

3) The yearly rate of seasonal allowances was decreased by 0.1 month's pay to 5.2 months' (currently 5.3 months' pay).

(Effective date: 1 April 1994)

(4) Child Care Leave Regulations

Effective 1 April of this year, regulations concerning child care leave were partially revised. As a result, employees are exempted from payment of their share of social insurance premiums during child care leave, and furthermore, child care leave allowance is payable to them. In the future, RERF will revise its related regulations based on agreement with the labor union.

(Effective date: 1 April 1995)

8. Election/appointment of directors and others (presented by Itsuzo Shigematsu)

\section{(1) Election of directors}

The resignation of Vice-Chairman Mortimer L Mendelsohn as of 3 July and the resignation of Permanent Director Donald $R$ Harkness (chief of research) as of 31 August were announced and approved. Seymour Abrahamson, professor at the zoology department, University of Wisconsin, was recommended and approved as successor to Dr Mendelsohn. It was 
agreed that he will concurrently serve as chief of research until Dr Harkness' successor is elected. Following announcement that the terms of three US directors, ie, Seymour Jablon, Warren K Sinclair and Joseph E Rall were due for expiration on 30 June, the reelection of $\mathrm{Mr}$ Jablon and $\mathrm{Dr}$ Sinclair and election of Patricia A Buffler, dean of the school of public health, University of California at Berkeley, as successor to Dr Rall were recommended and approved. It was also announced that the terms of three Japanese directors, ie, Kazuaki Arichi, Toshiyuki Kumatori and Teruhiko Saburi were due for expiration. Dr Arichi and Dr Kumatori were recommended for reelection and Masumi Oike, permanent director, National Social Insurance Societies Association was recommended for election, as successor to Dr Saburi. The two recommended actions were approved.

(2) Election of supervisors

David Williams and Akira Shishido, whose terms were due for expiration, were reelected. There are no restrictions in the reelection of supervisors.

\section{(3) Election of scientific councillors}

Three Japanese councillors, ie, Ei Matsunaga, Eisei Ishikawa, and Hiromichi Matsudaira, and two US councillors, ie, Arno G Motulsky and Leonard A Herzenberg, whose terms were due for expiration on 30 June, were reelected.

\section{(4) Appointment of operating committee members}

It has been agreed that the numbers of US and Japanese members of the Operating Committee be the same, but the position to which Katsutaro Shimaoka, who resigned in March last year, had been appointed still remains vacant. Thus, the US representation has been short by one member.

However, with the retirement of Akio A Awa, associate chief of research, as of 30 June, the numbers of the US and Japanese members will be the same and will satisfy the requirements of the Act of Endowment.

\section{(5) Appointment of chief of the Secretariat}

The Act of Endowment prescribes that chief of the Secretariat be appointed at the Board Meeting. There are no restrictions concerning the term. However, since the term of Yasukiyo Hirano, who was appointed at the Board Meeting last year, was for one year only, his reappointment was recommended and approved.

\section{Schedule of the next board meeting}

It was agreed to schedule the next meeting for three days from the 5-7 June 1996 at the Hiroshima Laboratory.

\section{Appended note}

At the afternoon session of this board meeting on 27 June, Dr Paul Seligman, deputy assistant secretary for health of the US Department of Energy, asked for 
permission to make an urgent report and, with the attendance of department chiefs specifically invited to the board meeting for the occasion, made the following announcement of the decision of DOE:

"Upon receipt of information that RERF accepted at this board meeting the recommendations made by the Scientific Council at its meeting last April, DOE has also decided to fully accept the recommendations. In this connection, it has also been decided to defer the action of terminating the grant DOE has awarded NAS for the management of RERF and to consider a further renewal of the grant for two years. Further, with approval of the recommendation of the Scientific Council for establishment of a blue ribbon committee by the board of directors of RERF, DOE intends to discuss the matter with MHW. The plan for training promising radiobiologists has received support from many quarters. Pending approval by Congress, DOE believes that the plan will lead to development of a very beneficial program in the future.

As for the future of RERF, unless the difficult financial and personnel problems RERF is now facing can be resolved, the possibility of carrying out the recommendations of the blue ribbon committee, if any, will be questioned. Nevertheless, DOE will continue to consider in a favorable light the efforts the directors and staff of RERF are making to the best of their ability."

Following Dr Seligman's announcement, Dr Hiroshi Maruyama, assistant director of the Planning Division of MHW, gave the position of MHW. MHW will discuss the question of establishing a blue ribbon committee with the RERF management and consider it in a forward-looking posture. As for the operational problems of RERF, an agreement has been reached with DOE to hold further discussions of the problems in the future. One point to bear in mind is the importance of maintaining intact the spirit of mutual trust RERF has with A-bomb survivors and the community at large. The question of transferring the grant of overseeing the management of RERF from NAS to elsewhere is a matter for the US to decide. However, MHW feels that a change of the grantee should not adversely affect the scientific excellence of the program. For this, MHW appreciates the decision DOE has made as reported.

Upon completion of discussion of all items on the agenda, Dr Shigematsu declared the meeting closed. 


\section{2nd Scientific Council Meeting}

19-21 April 1995

Auditorium, Radiation Effects Research Foundation

Hiroshima

\section{Agenda}

Day 1

Executive session Arno G Motulsky Ei Matsunaga

Greetings by RERF chairman Itsuzo Shigematsu

Briefing on NAS-RERF relationship, etc. Mortimor L Mendelsohn

Report from chief of research Donald R Harkness

\section{Research Information Center}

Overview

Jill L Ohara

\section{Departments of Statistics, Epidemiology, and Epidemiologic Pathology}

Overview: Statistics Dale L Preston

Overview: Epidemiology and Epidemiologic

Pathology ... Kiyohiko Mabuchi

A lesson from the LSS about low-dose studies Donald A Pierce

Report 12: Noncancer mortality Yukiko Shimizu

In utero exposure and brain damage Masanori Otake

Status of report on cancer incidence in the in utero cohort. Robert R Delongchamp

Prevalence of thyroid disorders in Nishiyama residents Yoshisada Shibata

Environmental/life style factors, radiation and mortality John B Cologne

\section{Department of Clinical Studies}

Departmental overview Kazunori Kodama

AHS health surveillance-pilot mail survey Saeko Fujiwara

Radiation and benign tumor-myoma uteri Sachiko Kawamura

Radiation and atherosclerosis-pulse wave velocity. Hironori Ueda

Psychological aspects of A-bomb survivors-progress report. Michiko Yamada 


\section{Department of Genetics}

Overview of the biochemical genetics study

and mutations in microsatellites Chiyoko Satoh

Quantitative analysis of bands on Southern filters Norio Takahashi

Molecular basis for genetic variants detected by computer analysis of 2-DE separation of genomic DNA Jun-ichi Asakawa

Overview of cytogenetics study and new dimension in individual dose reconstruction by ESR . Nori Nakamura

\section{Day 2}

\section{Department of Radiobiology}

Overview and GPA mutation study Mitoshi Akiyama

Abnormality of hematopoietic stem cells in A-bomb survivors Yuko Hirai

SCID-hu mouse model for human radiobiological studies. Seishi Kyoizumi

Molecular epidemiological analysis of liver cancer among A-bomb survivors Keisuke S Iwamoto

Molecular epidemiological analysis of skin cancer in A-bomb survivors................................ Terumi Mizuno

Characterization of SCID mutation and isolation of SCID complementing genes Masumi Abe

Interdisciplinary Session: Is there a genetic component to radiation-induced cancer?

Pedigree construction in the RERF study populations ..................................... John B Cologne

The potential for investigations at the molecular level. Charles E Land

Strategies for obtaining surgical and autopsy specimens. Kiyohiko Mabuchi

Molecular oncology at RERF: Future directions Toshio Seyama

Discussion: What are the opportunities and realities for approaching this problem at RERF?

Informal Sessions: Scientific councilors met with the individual research groups.

\section{Day 3}

Executive session (Closed)

Preparation of recommendations 
Council recommendations Arno G Motulsky

Ei Matsunaga

Closing remarks by RERF vice chairman Mortimer L Mendelsohn

Shigematsu and co-chairmen met with members of the press.

\section{Participants}

\section{Scientific Councillors}

Tomio Hirohata, professor of public health, Faculty of Medicine, Kyushu University

Eisei Ishikawa, professor emeritus, Jikeikai University School of Medicine

Hiromichi Matsudaira, former director general, National Institute of Radiological Sciences

Ei Matsunaga, professor emeritus, National Institute of Genetics

Shigefumi Okada, professor emeritus, University of Tokyo

Curtis C Harris, chief, Laboratory of Human Carcinogenesis, National Cancer Institute

Clark W Heath Jr, vice president for epidemiology and statistics, American Cancer Society

Leonard A Herzenberg, professor, Department of Genetics, Stanford University School of Medicine

John B Little, professor of radiobiology, Harvard University School of Public Health

Arno G Motulsky, professor of medicine and genetics, School of Medicine, University of Washington

\section{Observers}

Hiroshi Maruyama, assistant director, Planning Division Health Service Bureau, Ministry of Health and Welfare

Steve Galson, chief medical officer for Environment, Safety and Health, US Department of Energy (DOE)

Rika Maeshiro, special assistant of chief medical officer for Environment, Safety and Health, DOE

Timothy S Fox, RERF project manager, Office of Health, National Research Council, National Academy of Sciences (NAS)

Paul Gilman, executive director, Commission on Life Sciences, National Research Council, NAS

John D Zimbrick, director, Board on Radiation Effects Research, Commission on Life Sciences, National Research Council, NAS

Milton Eaton, DOE representative, US Embassy 
Charles E Land, health statistician, Radiation Epidemiology Branch, National Cancer Institute

Chris J Huyskens, executive officer, International Radiation Protection Association, Eindhoven University of Technology, Netherlands

John J Mulvihill, professor of human genetics, University of Pittsburgh

\section{Visiting directors}

Toshiyuki Kumatori, chairman, Radiation Effects Association

\section{Supervisor}

Akira Shishido, former director, National Institute of Health

\section{Directors}

Itsuzo Shigematsu, chairman

Mortimer L Mendelsohn, vice chairman

Donald R Harkness, permanent director and chief of research

Yutaka Hasegawa, permanent director

\section{General recommendations}

This year's meeting of the Science Council came at a period of great turmoil at the Radiation Effects Research Foundation (RERF). The US Department of Energy (DOE) decided to discontinue its contract with the US National Academy of Sciences (NAS) which has administered RERF for many years. The DOE will now be requesting American universities to compete for management of the US portion of the project's administration. The reasons for such a change appear to be complex. We perceive them to be related to a combination of circumstances, such as 1) DOE's wish to reassess the activities of RERF after many decades of work, 2) DOE's desire to strengthen its involvement in training of radiation biology specialists, and 3) DOE's responding with novel administrative arrangements at RERF in the face of Congressional desires to cut costs at all levels, particularly at DOE itself. The various developments together with the failure of DOE to communicate the full reasons for its decision led to strong reactions among RERF scientists and many other observers. We as scientific councilors are charged to ensure that the scientific activities of RERF are carried out properly, smoothly, and using the highest scientific standards. Radical changes in administrative managements are likely to affect the conduct of science and should be avoided unless carefully thought out and crafted.

The 50th anniversary of the atomic bombings occurs this year. We consider it appropriate and desirable that the scientific activities of RERF are assessed carefully and in depth by an outside expert group that has not been previously involved in RERF activities. We, therefore, recommend that a high-level international committee of distinguished scientists be appointed to carry out an assessment of RERF's current and 
future scientific activities. This committee should be impaneled under the sponsorship of an international body concerned with radiation biology, radiation effects on populations, and radiation protection. The panel should have representatives from Japan and the US, as well as from other countries. Even though only Japan and the US are directly concerned with RERF, we believe that RERF's mission is truly international and affects all of humankind. The newly established committee should pay particular attention to the nature and the extent of the studies to be carried out at RERF in the next 2 decades. Organizations such as the United Nations Scientific Committee on the Effects of Atomic Radiation, the International Commission on Radiological Protection, or the World Health Organization are possible sponsors, but other bodies may also be appropriate. A committee consisting of representatives of RERF, the US and Japanese sponsoring organizations (ie, the DOE and the Ministry of Health and Welfare), as well as selected radiation experts should be involved in setting up the membership of the distinguished ("blue ribbon") committee.

We welcome the idea of strengthening both training and scientific exchange activities of RERF. We therefore recommend that international postdoctoral fellowships and exchange programs be instituted to allow interested young researchers and more established scientists to work at RERF for various periods of time. We feel that the involvement of young scientists as well as of exchange scientists will lend strength to the scientific caliber of RERF's work. RERF should continue to be in the mainstream of radiation science and related fields. Enhancement of the training function and more exchange of scientists will help towards this goal.

Budgetary constraints are real and may get worse. It will therefore be increasingly difficult to fund all worthwhile research at RERF. We therefore strongly encourage RERF scientists to become more active in applying for research grants from various national and international organizations including those of Japan. In making these recommendations, we realize the existing constraint in funding with grants personnel positions in Japan.

We are concerned that the present turmoil about management may have deleterious effects on the research mission of RERF, particularly since negative newspaper reports about DOE's intentions-whether correct or not-may convince some atomic-bomb survivors to stop participating in ongoing and periodic studies.

We realize DOE's difficulties in funding a binational organization far away from the US and at the same time remain responsive to the current mood of the US Congress. Precipitous administrative moves, however, may seriously perturb difficult international relationships developed over many years. We are particularly concerned that RERF's binational character not be forgotten. This important aspect of RERF must always be considered in any future administrative moves.

We therefore recommend that DOE defer its current plan of discontinuing NAS sponsorship until the internationally distinguished committee has assessed RERF's total activities and has made its full report. We feel that such a step will defuse the 
current unrest among RERF's personnel, which is further accentuated by current fiscal uncertainties. This decision will have a favorable effect on the continuation of high-quality research at RERF. In this regard, we are particularly impressed by the excellent and improving scientific quality of RERF's work as presented during this meeting. RERF has a superb and unique cadre of fine scientists whose scientific activities must be maintained and fostered.

\section{Detailed recommendations Epidemiology and Statistics}

Work in epidemiology and statistics continues to make excellent contributions to RERF research, both in design and analysis of cohort studies and in collaborative efforts through a wide range of research investigations with other departments. The main components of this work involve 1) maintaining mortality and incidence data of several A-bomb survivor cohorts (LSS, in utero, $F_{1}$ ), as well as data from the Hiroshima and Nagasaki tumor registries; 2) analyzing updated information from those sources on a regular basis; and 3) designing and conducting specific studies related to radiation risk at particular cancer sites and in relation to nonradiation risk factors that may influence observed associations between radiation and disease. Despite present uncertainties regarding RERF resources and staffing, the Council strongly feels that these various tasks deserve highest priority and should not be allowed to falter.

Low-dose studies. A substantial proportion of A-bomb survivors received relatively low doses of radiation. These segments of the follow-up cohorts deserve particular attention in light of sustained worldwide interest in low-dose radiation health effects. A-bomb survivor analyses can be of particular value when viewed in relation to effects seen in higher dose categories. At the same time, findings of increased risk caused by low-dose irradiation must be interpreted with special caution because of particular susceptibility to distortion by even minor degrees of disease misclassification and the operation of competing risk factors. The presence of slight increases in excess relative risks (ERR) for solid-tumor mortality but their absence in incidence data may represent one aspect of this problem.

Noncancer mortality. In conducting analyses for Life Span Study Report 12, reanalysis of noncancer mortality in relation to Dosimetry System 1986 (DS86) dose have been conducted. An additional 5 years of mortality information have been added (now 1950-1990), and patterns of increasing risk with increasing dose continue to be seen for mortality since 1965: ERR values in the neighborhood of 1.0 at higher dose levels (4 Gy or more) with statistically significant dose trends for circulatory diseases, stroke, and digestive diseases. These data deserve continued close attention, since their public health importance (attributable risk) may be substantial, given the common nature of the disease categories involved. At present, however, any meaningful biologic interpretation is difficult, in view of the particular susceptibility of such low risk levels to influences of diagnostic misclassification and risk confounding. 
Although an assessment of such modifying factors appears to suggest only a minor impact on risk estimates, proof of the biologic reality of these low risks requires further independent observations at RERF (see below) and elsewhere, as well as laboratory data regarding the possible role of radiation in the etiology of arteriosclerotic and other diseases.

Brain damage in utero. Earlier RERF data regarding small head size in relation to in utero dose have been updated and supplemented with interesting analyses regarding subsequent patterns of mental retardation. Although sample sizes are small, the analyses are of great interest, especially in view of findings that suggest a dose threshold of about 0.20 Gy for severe retardation but not for other effects.

In utero cohorts. Considerable progress has been made in merging the two in utero cohorts and in clarifying the dosimetry status of the combined cohort. It is proposed to increase the sample further by adding those survivors exposed in early childhood. While such an addition will increase statistical power, it should not be viewed as a permanent analytic arrangement, given the differences in exposure conditions for pre- and post-natal states. The Council strongly endorses the close follow-up of survivors who were young at the time of the bombings and are now entering the age in which cancer risks increase.

Thyroid diseases. An interesting study has been conducted regarding thyroid diseases among persons exposed to fallout radiation in Nishiyama (near Nagasaki). Although the overall frequency of such diseases was not increased, certain categories of thyroid nodules were increased about threefold. The analyses appear to be complete, and future investigations are not necessary. It will be useful to compare these data with data from similar fallout surveys elsewhere in the world regarding the relationships between thyroid pathology and particular patterns of fallout radioactivity.

1965 LSS mail survey. Important data were reviewed from the 1965 Life Span Study (LSS) mail survey, which collected information (male only, ages 40-69 y) regarding various environmental and lifestyle risk factors that might effect estimates of LSS radiation risk. These data are particularly important in the context of interpreting the low levels of increased risk observed for certain solid cancers and for the wide range of noncancer mortality. All such sources of LSS and Adult Health Study (AHS) data deserve close examination, given the vulnerability of low excess relative risk values to distortion by such competing risk influences.

\section{Clinical Studies}

The important role of the AHS continues, both in terms of the regular clinical examination of cohort members and in relation to special investigations arising from AHS data collected over time. The Council would once again stress the central importance of AHS follow-up and study for the RERF mission and commends the staff for their continued excellent work. 
AHS pilot mail survey. In view of the aging nature of the AHS cohort, it has previously been urged that intensified efforts be employed to maintain close follow-up of cohort members, in the face of indications that follow-up tends to become more difficult as subjects age. The survey results strongly indicate that mailed questionnaires with follow-up contact, especially with nonresponders, are remarkably effective. Such methods deserve to be fully implemented as soon as possible. The overall success of AHS cohort work very much depends on a high degree of completeness in follow-up efforts and accurate recording of all health events as the population ages.

Uterine myomata. Earlier investigations regarding the possible relation of radiation dose to uterine myomata have been extended using more sensitive ultrasonic detection approaches for diagnosing tumor. The results continue to support a relationship between dose and tumor occurrence. The results are of considerable interest since only limited information exists regarding radiation as a cause of benign tumors. Perhaps information about menstrual histories may be relevant for future studies in this area.

Pulse-wave velocity. An interesting study was described whereby measurements of pulse-wave velocity were used as a surrogate for aortic atherosclerosis. The data are of interest since they represent an independent approach to the question of noncancer radiation effects. Whereas the observed association of velocity measurements to DS86 dose tends to support the LSS mortality observation, further studies using alternate techniques such as ultrasound measurements of carotid artery arteriosclerosis may be needed to explore this area of clinical research more fully.

Psychological studies. In response to Council comments in 1991 regarding evaluation of psychological effects on A-bomb survivors, work was undertaken to explore possible approaches for future studies and to reexamine results from such a survey conducted in the 1960s. The results presented support the concept of increased effects on survivors as a function of dose. It is unclear, however, to what extent such effects may arise from increased frequency of physical disorder in those higher dose categories. In view of present resource constraints, however, further investigations of this sort would be difficult and do not deserve a high priority.

Genetic subgroups. Extensive analyses have been conducted of LSS data concerning female breast-cancer incidence according to age at the time of the bombings (ATB), age of cancer diagnosis, and DS86 dose. The results strongly indicate greatly heightened radiation risk for women exposed and diagnosed at young ages; similar, but less extensive, data were also described for colon cancer. The findings correspond well with the concept that genetic high-risk groups exist for which early onset cancer may serve as a marker. Heightened radiation sensitivity among such persons, when exposed young, is of great potential importance. Future investigations of these subgroups to identify genetic subgroups with higher genetic susceptibility have a high priority. Although such studies should make use of molecular 
epidemiologic approaches, clinical genetic-epidemiologic work might well be undertaken at the same time to reconstruct family histories of cancer, which alone could be of great importance in further linking these LSS findings to underlying genetic mechanisms.

Pedigree construction. Continued progress was reported regarding identification of familial relationships among LSS cohort members. The task appears to be more complicated than originally envisioned since disparities in data available from different sources appear to be frequent. Computer programs have been devised to compare and merge family data from different files (master sample questionnaires and AHS records), and consideration is being given to use of koseki (family registry) data for further clarification of pedigrees. For the research purposes envisioned, however, it may not be necessary to conduct such complete merger and refinement of data but instead to focus efforts on confirming pedigree relationships in a sufficient number of subjects to support subsequent molecular genetic studies (see below). These efforts might focus particularly on sib-sib or parent-child pairs, using both koseki records, if accessible, as well as direct family interviews to confirm specific family relationships.

\section{Research Information Center}

In the face of increasing resource constraints, exceptional performance has been displayed in converting mainframe computers to personal-computer-based computing technology well ahead of schedule. Maintenance of equipment, continuous upgrading of computer programs, and ongoing staff training are essential if the new system is to realize its potential for supporting the various research efforts. Support for this essential aspect of RERF work has a high priority.

\section{Biochemical and molecular genetics}

An example of the high quality and productive research in molecular genetics as applied to the mission of RERF is the work carried out in Chiyoko Satoh's laboratory by Jun-ichi Asakawa and Mieko Kodaira. They have made the two-dimensional electrophoretic separation of restriction fragments of genomic DNA into an excellent method of detecting genetic variants, allowing differentially amplified regions of DNA, loss of heterozygosity, and other somatic or germ-line changes in DNA to be studied directly. They are also perfecting the reproducibility of gel patterns so that high-speed automated detection of quantitative as well as qualitative changes in fragment spots are possible.

This existing technique is beginning to be applied to DNA samples from mother/father/child trios for A-bomb survivors and other irradiated individuals. The methodology is also being explored to compare cancers with normal tissues and has much promise.

An important extension of the analytical power of detecting genetic or tissue-specific differences is molecular cloning of variants so that these can be 
sequenced, mapped, and otherwise characterized. This is an example of sophisticated research that provides, through collaboration with at least one university, dissemination of new knowledge and at the same time helps in training other scientists in these new techniques.

These kinds of activities are strongly encouraged by the Science Council. Additional funding for this kind of "cutting edge" research should be sought from outside grants and contracts. We believe funding agencies such as those dealing with cancer prevention and treatment would find this work of great interest for their mission.

\section{Biodosimetry}

Major progress has been made by the cytogenetics group headed by Nori Nakamura during the last year. Cytogenetic examinations of Hiroshima survivors using the fluorescence in-situ hybridization (FISH) method started in April 1994 on a routine basis. This is a most efficient technique of biodosimetry.

An unexpected finding among exposed subjects with chromosomal deletions was a cluster of interstitial deletion on the long arm of chromosome 5, and the correlation of terminal deletions with chromosome length.

The Council was interested in the result of electron spin resonance (ESR) measurement using tooth enamel which Dr Nakamura had done in collaboration with an outside scientist, who made equipment for ESR measurement available. The results of ESR analysis for known chromosome aberrations with known DS86 doses suggest that this technique could be used as a new means of biologic dosimetry, although availability of tooth samples may be limited. However, Dr Nakamura has collected over 300 teeth samples from A-bomb survivors, and an ESR machine has recently been installed in his laboratory. The Council encourages the establishment of these ESR measurements in the near future.

\section{Molecular epidemiology studies}

The molecular pathogenesis of diseases associated with ionizing radiation is an exciting and expanding area of biomedical research. In recent years, the RERF staff has acquired skills in molecular biology and developed effective strategies for conducting investigations in the molecular epidemiology of cancer. Pilot studies have already produced interesting results. An increased frequency of RET protooncogene mutations in thyroid cancers from A-bomb survivors, as well as among children living in areas contaminated by the Chernobyl accident, and an altered mutation spectrum of the p53 tumor suppressor gene in basal-cell carcinoma in both sun-exposed and nonexposed skin of A-bomb survivors are interesting examples. The molecular analysis of unusual cancer subpopulations can also yield far reaching results. One example is the analysis of germline mutations in the BRCA1 and other cancer susceptibility genes occurring in young women with early onset breast cancer who were exposed to ionizing radiations 
before or during puberty. A comparison between the molecular "fingerprints" of cancer-related genes associated with ionizing radiation and those produced by other carcinogens is an important area for future investigation.

RERF should focus its limited molecular epidemiology resources on studies to test hypotheses tightly linked to its ongoing clinical and epidemiological studies of $A$-bomb survivors. Collaborations with colleagues in local, national, and international institutions are encouraged to leverage resources. The results from these high-priority molecular epidemiology studies of A-bomb survivors should lead to new insights in radiation carcinogenesis and generate hypotheses for future mechanistic studies.

\section{Radiobiology of somatic mutations and molecular oncology}

Mitoshi Akiyama provided an overview of the major projects in the radiobiology program, focusing in particular on the somatic mutation and molecular oncological studies in A-bomb survivors. Among the former, he described substantial progress in studies of mutation in the glycophorin-A (GPA) and T-cell receptor (TCR) loci of blood cells. A promising new approach is the molecular analysis of GPA mutants in reticulocytes. Molecular studies of FACS-sorted mutants permitted identification of the precise molecular changes and radiation dose dependence of these changes in A-bomb survivors and irradiated patients. Similar cellular and molecular level assays of radiation dose dependency were done with T-cell receptor and HPRT loci using separation by flow cytometry and polymerase chain reaction (PCR) methods. These projects appear to be progressing well and should provide valuable information concerning the ability to predict carcinogenic effects of radiation in humans by use of mutation assays in their somatic cells.

There appear to be minor correlations between age and smoking and glycophorin mutations, as well as with city (Hiroshima $>$ Nagasaki). There are also significant, yet unexplained, sex differences (male $>$ female), that are greater than the effect of smoking. These trends seem to correlate with those for background solid cancers. The interactive effects between ionizing radiation and other causes of cancer are important in human cancer risk assessment. Therefore, the possible interactive effects between radiation exposure and tobacco smoking on GPA mutation frequency in the cancer group as compared with the noncancer group should be examined. In addition, the strikingly lower GPA mutation frequency in females compared to males generates several hypotheses including the possible effects of sex hormones, on either hemopoietic stem cell proliferation rates or survival of GPA mutant cells.

Overall, GPA mutations appear to be a good somatic-cell indicator of radiation damage that correlates with the risk of developing solid tumors; the doubling dose for GPA mutations was 1.2 Sv as compared with $1.59 \mathrm{~Sv}$ for solid tumors. Whereas GPA mutant frequency reflects the life-time accumulation of mutations, the half-life of T-cell receptor (TCR) mutations is only $2-3$ years. Therefore, the TCR assay is of value as a biological dosimeter of recent radiation exposure. The doubling doses appear to be 
much lower than for GPA mutants. Increased TCR mutations in a few A-bomb survivors appear to represent clonal expansion. These studies of TCR mutations as a somatic-cell assay for recent radiation exposure should be continued.

Stem-cell studies showed that mutations at the hematopoietic stem-cell stage, were generally stable over many years thus serving as biological dosimeters for A-bomb survivors and other types of irradiation.

The SCID-hu mouse model for human epilation is of considerable mechanistic interest since few such experimental studies have been carried out. These results contribute significantly to understanding of this radiobiological phenomenon. Additional more-basic studies of epilation including the mechanism of radioresistance of follicular stem cells would be appropriate for independent grant support. This SCID model may have important application in areas such as studies of basal cell carcinoma.

Overall, the work of the radiobiology group and collaborators is an excellent example of scientific excellence of direct relevance to the RERF mission. The extensive use of stored material and cell lines established from A-bomb survivors and only available at RERF was noted and emphasizes this unique resource.

We encourage Dr Akiyama's group to continue with the general thrust of this work.

\section{Genes responsible for SCID mutation(s)}

This work aimed at identifying the responsible mouse genes for radiosensitivity and complementing these with human genes conferring resistance, was unusual in being carried out independently of other similar studies. Masumi Abe localized the responsible region to human chromosome 8 . Others have moved faster in actually identifying the human and mouse genes. With the pending departure of $\mathrm{Dr}$ Abe, termination of this program at RERF seems appropriate. 


\section{Directors, Supervisors, and Scientific Councilors}

\section{As of 31 March 1996}

\section{Permanent Directors}

Chairman: Itsuzo Shigematsu, former director, Department of Epidemiology, Japanese National Institute of Public Health

Vice chairman (chief of research): Seymour Abrahamson, professor, Zoology Department, University of Wisconsin

Permanent director: Donald R Harkness, professor, Department of Medicine, University of Wisconsin

Permanent director: Yutaka Hasegawa, former director, Chugoku-Shikoku Regional Medical Affairs Bureau, Japanese Ministry of Health and Welfare

\section{Directors}

Kazuaki Arichi, executive director, Japan Institute of International Affairs

Masumi Oike, permanent director, National Social Insurance Societies Association

Toshiyuki Kumatori, chairman, Radiation Effects Association

Seymour Jablon, former expert, Radiation Epidemiology Branch, Division of Cancer Etiology, US National Cancer Institute

Patricia A Buffler, dean and professor of epidemiology, School of Public Health, University of California, Berkeley

Warren K Sinclair, president emeritus, US National Council on Radiation Protection and Measurements

\section{Supervisors}

Akira Shishido, former director, Japanese National Institute of Health, Ministry of Health and Welfare

David Williams, senior financial advisor, US National Academy of Sciences

\section{Scientific Councilors}

Tomio Hirohata, professor of public health, Kyushu University Faculty of Medicine

Eisei Ishikawa, professor emeritus, Jikeikai University School of Medicine Hiromichi Matsudaira, chairman, Research Development Corporation of Japan Ei Matsunaga, professor emeritus, Japanese National Institute of Genetics Shigefumi Okada, professor emeritus, University of Tokyo 
Curtis C Harris, chief, Laboratory of Human Carcinogenesis, US National Cancer Institute

Clark W Heath $\mathrm{Jr}$, vice president for epidemiology and statistics, American Cancer Society

Leonard A Herzenberg, professor of genetics, Stanford University School of Medicine

John B Little, professor of radiobiology, Harvard University

Arno G Motulsky, professor of medicine and genetics, School of Medicine, University of Washington

\section{Consultants}

\section{As of 31 March 1996}

\section{Department of Clinical Studies}

Shigetoshi Antoku, professor, Department of Experimental Radiology, Kyushu University Faculty of Medicine

Kanji Choshi, professor, Department of Ophthalmology, Hiroshima University School of Medicine

Jess D Curb, professor, John A Burns School of Medicine, University of Hawaii

Haruo Ezaki, Hiroshima Thyroid Clinic

Kohei Hara, former professor, 2nd Department of Internal Medicine, Nagasaki University School of Medicine

Shuichi Hatano, professor, Faculty of Social Welfare, Shukutoku University

Kuniaki Hayashi, professor, Department of Radiology, Nagasaki University School of Medicine

Yutaka Hosoda, chief, Radiological Epidemiology Center, Radiation Effects Association

Michito Ichimaru, director, Sasebo Dojinkai Hospital

Chikako Ito, deputy director, Health Management and Promotion Center, Hiroshima Atomic Bomb Casualty Council

Katsuhide Ito, professor, Department of Radiology, Hiroshima University School of Medicine

Motomori Izumi, vice chairman, Nagasaki Prefectural Saiseikai Hospital

Tadashi Kawai, professor, Department of Clinical Pathology, Jichi Medical School Hospital

Atsushi Kuramoto, director, Hiroshima General Hospital

Shigenobu Nagataki, dean, Nagasaki University School of Medicine

Shigenobu Nakamura, professor, 3rd Department of Internal Medicine, Hiroshima University School of Medicine 
Yutaka Okumura, professor, Department of Radiobiophysics, Atomic Disease Institute, Nagasaki University School of Medicine

Kozo Ohama, professor, Department of Obstetrics and Gynecology, Hiroshima University School of Medicine

Hajime Orimo, professor, Department of Geriatrics, Tokyo University Faculty of Medicine

Shozo Sawada, professor, Hiroshima Prefectural Health and Welfare College

Masao Tomonaga, professor, Department of Sequela Therapy, Atomic Disease Institute, Nagasaki University School of Medicine

Mitsuhiro Tsujihata, director, Nagasaki North Hospital

Toshiaki Usui, principal, Nagasaki Prefectural Health Care School

Lon R White, director, Honolulu Heart and Aging Studies

Michio Yamakido, professor, 2nd Department of Internal Medicine, Hiroshima University School of Medicine

Shoso Yamamoto, professor, Department of Dermatology, Hiroshima University School of Medicine

Shun-ichi Yamashita, professor, Department of Preventive Medicine, Atomic Disease Institute, Nagasaki University School of Medicine

Hiroshi Yanagawa, chairman and professor, Department of Public Health, Jichi Medical School

Keisuke Yano, professor, 3rd Department of Internal Medicine, Nagasaki University School of Medicine

Koji Yoshizawa, professor, Department of Hygiene, Hiroshima University School of Medicine

\section{Department of Genetics}

Joe B Gray, professor, Department of Laboratory Medicine, University of California, San Francisco

Tasuku Honjo, professor, Department of Biochemistry, Kyoto University Faculty of Medicine

Leonard S Lerman, senior lecturer, Massachusetts Institute of Technology

Yoshiyuki Sakaki, professor, Human Genome Analysis Center, Institute of Medical Science, University of Tokyo

Masao Sasaki, chief and professor, Radiation Biology Center, Kyoto University

Eiichi Soeda, senior research scientist, Institute of Physical and Chemical Research, Wakoshi, Saitama

Michihiro Yoshida, professor, Chromosome Research Unit, Faculty of Science, Hokkaido University 


\section{Department of Radiobiology}

Michael A Bean, director, Pacific Northwest Research Foundation, Seattle, Washington

Max D Cooper, professor, Howard Hughes Medical Institute, Birmingham, Alabama

Kiyohiko Dohi, professor, 2nd Department of Surgery, Hiroshima University School of Medicine

Akihiro Itoh, professor, Research Institute for Nuclear Medicine and Biology, Hiroshima University

Yasushi Okumura, professor, Department of Immunology, Juntendo University School of Medicine, Tokyo

Koichi Tatsumi, chief, Department of Biostatistics, National Institute of Radiological Sciences, Chiba

\section{Department of Epidemiology}

Suminori Akiba, professor, Department of Public Health, Kagoshima University Faculty of Medicine

Takashi Aoyama, professor, Experimental Radiology, Shiga University of Medical Science

Takeshi Hirayama, director, Institute of Preventive Oncology, Tokyo

Takayoshi Ikeda, professor, 1st Department of Pathology, Nagasaki University School of Medicine

Koki Inai, professor, 2nd Department of Pathology, Hiroshima University School of Medicine

Hideyo Itakura, professor, Department of Pathology, Institute for Tropical Medicine, Nagasaki University

Nanao Kamada, professor, Research Institute for Nuclear Medicine and Biology, Hiroshima University

Hiroo Kato, former director general, National Institute for Minamata Disease

Takashi Maruyama, head, Division of Planning, Radiation Effects Association

Koji Namba, professor, Hiroshima University Faculty of Integrated Arts and Sciences

Otsura Niwa, professor, Research Institute for Nuclear Medicine and Biology, Hiroshima University

Takashi Saku, professor, Department of Oral Pathology, Niigata University School of Dentistry

William J Schull, director and professor, Graduate School of Biomedical Sciences, Center for Demographic and Population Studies, University of Texas

Ichiro Sekine, professor, Department of Pathophysiology, Atomic Disease Institute, Nagasaki University School of Medicine 
Takashi Taguchi, professor, 2nd Department of Pathology, Nagasaki University School of Medicine

Eiichi Tahara, professor, 1st Department of Pathology, Hiroshima University School of Medicine

Kazuo Uemura, dean, Ryutsu Keizai University, Ryugasaki, Ibaraki

\section{Department of Statistics}

Yasunori Fujikoshi, professor, Department of Mathematics, Hiroshima University Faculty of Science

Akio Kudo, professor, Department of Economic Information, Hyogo University Sumiyasu Yamamoto, director, Kake International Institute for Natural Sciences, International Center for Academic Exchange, Kurashiki, Okayama

Takashi Yanagawa, professor, Department of Mathematics, Kyushu University Faculty of Science

\section{Information Technology Department}

Harvard H Holmes, deputy head, Computing Science Research Programs, Computing Services Department, Lawrence Berkeley Laboratory

George D Kerr, senior research scientist, Health and Safety Research Division, Oak Ridge National Laboratory

Thomas R Slezak, computer scientist, Human Genome Center, Lawrence Livermore National Laboratory

\section{Secretariat}

Akio Awa, former associate chief of research, RERF

Hisashi Kodama, director, Hiroshima Central Health Management Clinic, Nippon Telegraph and Telephone Corporation

Shoji Tokuoka, former senior consulting scientist, RERF

\section{Expert Advisors}

\section{As of 31 March 1996}

\section{Department of Clinical Studies}

Hiroo Dohi, chief, 4th Department of Internal Medicine, Hiroshima Red CrossA-bomb Hospital

Kingo Fujimura, assistant professor, Research Institute for Nuclear Medicine and Biology, Hiroshima University

Motonobu Hayano, assistant professor, 3rd Department of Internal Medicine, Nagasaki University School of Medicine 
Ryoji Hirose, chief, Division of Dermatology, Nagasaki Central National Hospital

Masaki Moriyama, assistant professor, Department of Hygiene, Nagasaki University School of Medicine

Shizuyo Kusumi, department chief, Radiological Epidemiology Investigation Center, Radiation Effects Association

Hideo Sasaki, department chief, Health Management and Promotion Center, Hiroshima Atomic Bomb Casualty Council

\section{Department of Genetics}

Rork Kuick, computer scientist, Department of Pediatrics, University of Michigan Medical School

\section{Department of Epidemiology}

Hiroya Egawa, chief, Department of Internal Medicine and Pathology, Hiroshima Municipal Asa Citizens Hospital

Megumu Fujihara, chief, Department of Pathology, Hiroshima Red Cross-Abomb Hospital

Hideharu Fujii, chief, Division of Clinical Laboratories, Nagasaki Chuo National Hospital

Toshiyuki Fukuhara, chief, Department of Pathology, Hiroshima Prefectural Hospital

Aya Hanai, supervisor, Epidemiology and Investigation Section, Osaka Prefectural Center for Adult Diseases

Yuzo Hayashi, vice chairman and senior chief, Department of Clinical Laboratories, Hiroshima Asa Citizens Hospital

Michinori Kabuto, Regional Environmental Study Group, National Institute for Environmental Studies, Tsukuba, Ibaraki

Kioko Kawai, medical chief, Department of Pathology, Nagasaki Citizens Hospital Masao Kishikawa, assistant professor, Medical Materials Center for Atomic Bomb Casualty, Atomic Disease Institute, Nagasaki University School of Medicine

Takeshi Matsuo, chief, Pathological Laboratory, Nagasaki General Health Center Hiroo Matsuura, chief, Department of Pathology, Hiroshima Citizens Hospital Elaine Ron, research scientist, US National Cancer Institute, Bethesda, Maryland

Osamu Takahara, chief, 2nd Department of Clinical Laboratories, Nagasaki Red Cross-A-bomb Hospital

Nobuo Tsuda, assistant professor, Department of Clinical Laboratories, Nagasaki University School of Medicine 


\section{Department of Statistics}

Richard Sposto, associate professor of research, Department of Preventive Medicine, School of Medicine, University of Southern California, and Children's Cancer Group, Arcadia, California

Daniel $O$ Stram, assistant professor, Department of Preventive Medicine, School of Medicine, University of Southern California, and Children's Cancer Group, Arcadia, California

Michael Vaeth, associate professor, University of Århus, Århus, Denmark

\section{Information Technology Department}

Reiji Aibara, assistant professor, Research Center for Integrated Systems, Hiroshima University

\section{Operating Committee}

As of 31 March 1996

Operating Committee chairman

Itsuzo Shigematsu, RERF chairman

\section{Members}

Seymour Abrahamson, vice chairman and chief of research

Yutaka Hasegawa, permanent director

Yasukiyo Hirano, chief of the Secretariat

Akio Awa, RERF consultant

Jill L Ohara, chief, Information Technology Department

Richard D Sperry, advisor of the Secretariat

Tadashi Nakaoka, chief of administration, Secretariat, Nagasaki Laboratory 


\section{Standing Committees}

As of 31 March 1996

\section{Research Protocol Review Committee}

\section{Committee chairman}

Seymour Abrahamson, chief of research

\section{Committee vice chairman}

Nori Nakamura, assistant chief, Department of Genetics

\section{Members}

Shoji Tokuoka, consultant

Kazunori Kodama, chief, Department of Clinical Studies

Chiyoko Satoh, chief, Department of Genetics

Mitoshi Akiyama, chief, Department of Radiobiology

Toshio Seyama, assistant chief, Department of Radiobiology

Kiyohiko Mabuchi, chief, Department of Epidemiology

Dale L Preston, chief, Department of Statistics

Midori Soda, assistant chief, Department of Epidemiology, Nagasaki Laboratory

\section{Executive secretary}

Akiko Enami, chief, Directors' Office, General Affairs Section, Secretariat

\section{Scientific Reports Review Committee \\ Committee chairman}

Seymour Abrahamson, vice chairman

\section{Deputy chairman}

Yutaka Hasegawa, permanent director

\section{Members}

Shoji Tokuoka, consultant

Jun-ichi Asakawa, senior scientist, Biochemical Genetics Laboratory, Department of Genetics

Keisuke S Iwamoto, research scientist, Cell Biology Laboratory, Department of Radiobiology

John B Cologne, associate senior scientist, Department of Statistics 


\section{Executive secretary}

Beth A Magura, chief, Editorial and Publications Section, Publication and Documentation Center

\section{Human Investigation Committee \\ Committee chairman}

Yutaka Hasegawa, permanent director

\section{Deputy chairman}

Kiyohiko Mabuchi, chief, Department of Epidemiology

\section{Members}

Toshio Hisayuki, lawyer

Fumio Kanazawa, professor, Law and Economic Faculty, Okayama College of Commerce

Saeko Fujiwara, assistant chief, Department of Clinical Studies

Mimako Nakano, research scientist, Cytogenetics Laboratory, Department of Genetics

Yuko Hirai, senior scientist, Immunology Laboratory, Department of Radiobiology Yasuhiko Yoshimoto, chief and senior scientist, Epidemiologic Analysis Laboratory, Department of Epidemiology

Tetsuo Imada, assistant chief, Information Technology Department

Takeo Murata, assistant chief, Secretariat

Yoshisada Shibata, chief, Department of Epidemiology, Nagasaki Laboratory

\section{Executive secretary}

Kanjuro Hidaka, chief, Clinical Contacting Section, Department of Clinical Studies

\section{Library Committee}

\section{Committee chairman}

Jill L Ohara, chief, Information Technology Department

\section{Members}

Michiko Yamada, associate senior scientist, Division of Medicine, Department of Clinical Studies

Chiyoko Satoh, chief, Department of Genetics

Toshio Seyama, assistant chief, Department of Radiobiology

Yukiko Shimizu, assistant chief, Department of Epidemiology

Tetsuo Imada, assistant chief, Information Technology Department 
Beth A Magura, chief, Editorial and Publications Section, Publication and Documentation Center

Yoshisada Shibata, chief, Department of Epidemiology, Nagasaki Laboratory

\section{Executive secretary}

Ai Yokoyama, assistant chief, Library Section, Information Technology Department

\section{Public Relations Committee}

\section{Committee chairman}

Kazunori Kodama, chief, Department of Clinical Studies

\section{Deputy chairman}

Nori Nakamura, assistant chief, Department of Genetics

\section{Members}

Takeo Murata, assistant chief, Secretariat, Hiroshima Laboratory

Tadashi Nakaoka, chief of administration, Secretariat, Nagasaki Laboratory

Sadayuki Ban, associate senior scientist, Cell Biology Laboratory, Department of

Radiobiology

Shoichiro Fujita, chief, Statistical Analysis Laboratory, Department of Statistics

\section{Executive secretary}

Shun-ichiro Matsuoka, chief, Administration and Support Section, Publication and Documentation Center

\section{Professional and Supervisory Staff}

\section{As of 31 March 1996}

Seymour Abrahamson, chief of research

\section{Hiroshima Laboratory}

\section{Department of Clinical Studies}

Kazunori Kodama, department chief

Saeko Fujiwara, assistant department chief

Ken-ichiro Ishii, administrative assistant department chief

Noboru Ueda, attached to the department 


\section{Division of Medicine}

Kazuo Neriishi, division chief

Michiko Yamada, associate senior scientist

Masaharu Nobuyoshi, research scientist

Hironori Ueda, research scientist

\section{Division of Radiology}

Kazunori Kodama, acting division chief

Hiromichi Fukuchi, chief of technicians

Yumiko Sano, assistant chief of technicians

\section{Division of Clinical Laboratories}

Saeko Fujiwara, acting division chief (assistant department chief)

Ken-ichiro Ishii, acting chief of technicians

\section{Nursing Section}

Toyoko Nakamura, chief of nurses

Yayoko Hirofuji, assistant chief of nurses

\section{Clinical Administration Section}

Iwao Osaki, section chief

Miyoko Hidaka, assistant section chief

\section{Clinical Contacting Section}

Kanjuro Hidaka, section chief

Midori Kamouchi, assistant section chief

Naoya Kagimoto, assistant section chief

\section{Department of Genetics}

Chiyoko Sato, department chief (senior scientist)

Nori Nakamura, assistant department chief

Hideko Murakami, visiting research scientist

\section{Cytogenetics Laboratory}

Yoshiaki Kodama, laboratory chief

Kazuo Ohtaki, research scientist

Mimako Nakano, research scientist

\section{Biochemical Genetics Laboratory}

Norio Takahashi, laboratory chief

Jun-ichi Asakawa, senior scientist

Mieko Kodaira, associate senior scientist

Junko Kaneko, assistant chief of technicians 


\section{Department of Radiobiology}

Mitoshi Akiyama, department chief

Toshio Seyama, assistant department chief

\section{Cell Biology Laboratory}

Toshio Seyama, acting laboratory chief

Sadayuki Ban, associate senior scientist

Terumi Mizuno, research scientist

Keisuke S Iwamoto, research scientist

\section{Immunology Laboratory}

Seishi Kyoizumi, laboratory chief

Yuko Hirai, senior scientist

Yoichiro Kusunoki, associate senior scientist

Tomonori Hayashi, research scientist

Takako Suzuki, research scientist

Masaharu Nobuyoshi, research scientist (concurrent assignment)

Noriko Abe, chief of technicians

Kazumi Tanabe, assistant chief of technicians

\section{Department of Epidemiology}

Kiyohiko Mabuchi, department chief

Yukiko Shimizu, assistant department chief (senior scientist)

Yasuyuki Fujita, assistant department chief

Yasuhiko Yoshimoto, chief, Epidemiologic Analysis Laboratory (senior scientist)

Donald A Pierce, senior scientist (concurrent assignment)

Charles E Land, visiting research scientist

Shigeyoshi Michitsuji, administrative assistant department chief

\section{Master File Section}

Morito Dote, section chief

Kyoko Katagami, assistant section chief

Masashi Sakurai, assistant section chief

\section{Pathology Laboratory}

Yasuyuki Fujita, acting laboratory chief

Masayoshi Tokunaga, visiting research scientist

Tsunemaro Omoto, section chief

Yukinobu Nakata, assistant section chief (concurrent assignment)

Chiyako Omoto, assistant chief of technicians 


\section{Tumor \& Tissue Registry Office}

Yasuyuki Fujita, acting office chief

Nobuko Hoshino, acting section chief

Yukinobu Nakata, assistant section chief

\section{Department of Statistics}

Dale L Preston, department chief

Masanori Otake, assistant department chief

Shoichiro Fujita, chief, Statistical Analysis Laboratory (senior scientist)

Eiji Nakashima, research scientist

John B Cologne, associate senior scientist

Fumiyoshi Kasagi, research scientist

Robert R Delongchamp, research scientist

Donald A Pierce, senior scientist

\section{Information Technology Department}

Jill L Ohara, department chief

Tetsuo Imada, administrative assistant department chief

Timothy Demarest, research scientist

\section{Systems Technology Section}

Tsuneyuki Isaka, section chief

Mieko Koda, assistant section chief

\section{Library Section}

Jill L Ohara, acting section chief

Ai Yokoyama, assistant section chief

\section{Publication and Documentation Center}

Kimiko Ono, acting center chief

\section{Administration and Support Section}

Shun-ichiro Matsuoka, section chief

Tokiko Himori, assistant section chief

Eiko Ishizaki, assistant section chief

\section{Editorial and Publications Section}

Beth A Magura, section chief

Junso Takayama, assistant section chief

Reiko Sasaki, assistant section chief 


\section{Radioisotope Facility}

Norio Takahashi, division chief (concurrent assignment)

\section{Secretariat}

Yasukiyo Hirano, chief

Takeo Murata, assistant chief of Secretariat

Richard D Sperry, advisor

Shizuo Inoue, advisor

\section{General Affairs Section}

Takeshi Abe, section chief

Akiko Enami, chief, Directors' Office

Tadaaki Watanabe, assistant section chief

\section{Personnel Section}

Hiroyuki Tominaga, section chief

Jun-ichi Nakamura, assistant section chief

Fujiko Naito, assistant section chief

\section{Accounting Section}

Torao Sasaki, section chief

Akio Ihara, assistant section chief

\section{Supply and Property Section}

Yofu Sato, section chief

Kayoko Arakawa, assistant section chief

Toshikazu Ohmori, assistant section chief

\section{Nagasaki Laboratory}

\section{Department of Clinical Studies}

Masazumi Akahoshi, acting department chief

Yasutaka Ohgushi, administrative assistant department chief

\section{Division of Medicine}

Masazumi Akahoshi, acting division chief

Midori Soda, research scientist (concurrent assignment)

Tomoko Nishikawa, research scientist (concurrent assignment)

\section{Division of Radiology}

Masazumi Akahoshi, acting division chief

Ichiro Koba, chief of technicians 
Division of Clinical Laboratories

Tomoko Nishikawa, acting division chief

Nobuaki Taira, assistant chief of technicians

Clinical Administration Section

Yasutaka Ogushi, acting section chief

Koki Sonoda, assistant section chief

Clinical Contacting Section

Nachiko Fukae, section chief

Noboru Imamichi, assistant section chief

Department of Radiobiology

Yutaka Hasegawa, acting department chief

Cell Biology Laboratory

Kiyohiro Hamatani, laboratory chief

Masahiro Ito, research scientist

Hiromitsu Miki, assistant chief of technicians

Department of Epidemiology

Yoshisada Shibata, department chief

Midori Soda, assistant department chief

Koji Nakamura, administrative assistant department chief

Master File Section

Osamu Kusumi, section chief

Toshiko Tanaka, assistant section chief

\section{Pathology Laboratory}

Midori Soda, acting laboratory chief

Masayoshi Tokunaga, visiting research scientist (concurrent assignment)

Yoko Yanagihara, chief of technicians

Tumor and Tissue Registry Office

Midori Soda, acting office chief

Yuiko Imamichi, section chief

Makiko Hamamatsu, assistant section chief

Computer Management Office

Yoshisada Shibata, acting office chief 


\section{Secretariat}

Tadashi Nakaoka, chief of administration

\section{General Affairs Section}

Takaaki Hashiguchi, section chief

Kin-ichi Honda, chief of Public Relations Office

Hiroshi Ichoda, assistant section chief

Atsuko Nakagawa, assistant section chief

\section{Accounting Section}

Mitsuo Yutaka, section chief

Yasushi Takeda, assistant section chief 


\section{Summary Tables}

Contacting Adult Health Study (AHS) participants and contacting families for permanent lymphocyte cell-line ${ }^{\star}$ establishment

April 1995-March 1996

\begin{tabular}{lcccc}
\hline City & $\begin{array}{c}\text { AHS } \\
\text { allocated for } \\
\text { contacting }\end{array}$ & $\begin{array}{c}\text { Appointments } \\
\text { made }\end{array}$ & $\begin{array}{c}\text { PLC family } \\
\text { allocated for } \\
\text { contacting }\end{array}$ & $\begin{array}{c}\text { Appointments } \\
\text { made }\end{array}$ \\
\hline Hiroshima & 11,225 & 1788 & 70 & $3(10)$ \\
Monthly average & 935.4 & 149 & 5.8 & $0.3(0.8)$ \\
Nagasaki & 6873 & 1119 & 97 & $8(20)$ \\
Monthly average & 572.7 & 93.3 & 8.1 & $0.7(1.7)$ \\
\hline
\end{tabular}

*RP 5-85: Culture of permanent lymphocyte cell lines (PLC) as sources of biological samples for investigation of genetic effects of radiation on children of atomic-bomb survivors. The study was initiated in Hiroshima in August 1985 and commenced in Nagasaki in September 1986.

Note: The numbers of contacts made are entered in the columns "AHS allocated for contacting" and "PLC family allocated for contacting." The column "Appointments made" for PLC study gives the numbers of families. Figures in parentheses are the numbers of persons examined.

Medical clinic visits

April 1995-March 1996

\begin{tabular}{|c|c|c|c|c|c|c|}
\hline \multirow[b]{2}{*}{ City } & \multirow{2}{*}{$\begin{array}{c}\text { Total } \\
\text { persons }\end{array}$} & \multicolumn{2}{|c|}{ AHS } & \multicolumn{2}{|c|}{ Non-AHS } & \multirow{2}{*}{$\begin{array}{c}\text { Specimens } \\
\text { only }\end{array}$} \\
\hline & & Scheduled & Others* & Referral & Return & \\
\hline Hiroshima & 2336 & 1788 & 365 & 68 & 0 & 115 \\
\hline Monthly average & 194.7 & 149 & 30.4 & 5.7 & 0 & 9.6 \\
\hline Nagasaki & 1507 & 1119 & 273 & 38 & 9 & 68 \\
\hline Monthly average & 125.6 & 93.3 & 22.7 & 3.2 & 0.8 & 5.7 \\
\hline
\end{tabular}

*Return visit, nonscheduled examination, etc.

Note: AHS = Adult Health Study. "Non-AHS" refers to the family members, friends, etc, of AHS participants. "Specimens only" refers to the collection of urine, stool, sputum, etc from examinees who do not come to the clinic. 
Cryopreservation of blood samples obtained from AHS participants

January-December 1995

\begin{tabular}{lccc}
\hline & \multicolumn{2}{c}{ Number of survivors by age at the time of bombing* } \\
\cline { 2 - 4 } DS86(Gy) & $0-15 y$ & \multicolumn{1}{c}{ Total } \\
\hline & \multicolumn{3}{c}{ Hiroshima } \\
\cline { 2 - 4 } $0-0.5$ & $513(228,285)$ & $737(187,550)$ & $1250(415,835)$ \\
$\geq 0.5$ & $170(75,95)$ & $260(68,192)$ & $430(143,287)$ \\
Unknown & $36(7,29)$ & $112(32,80)$ & $148(39,109)$ \\
Total & $719(310,409)$ & $1109(287,822)$ & $1828(597,1231)$ \\
& & Nagasaki & \\
$0-0.5$ & $225(106,119)$ & $342(114,228)$ & $567(220,347)$ \\
$\geq 0.5$ & $125(52,73)$ & $226(73,153)$ & $351(125,226)$ \\
Unknown & $71(33,38)$ & $205(73,132)$ & $276(106,170)$ \\
Total & $421(191,230)$ & $773(260,513)$ & $1194(451,743)$ \\
\hline
\end{tabular}

*Total number of persons: (male, female)

April 1990-December 1995

\begin{tabular}{lccc}
\hline & \multicolumn{3}{c}{ Number of survivors by age at the time of bombing* } \\
\cline { 2 - 4 } DS86 (Gy) & $0-15 y$ & Total \\
\hline & \multicolumn{4}{c}{ Hiroshima } \\
\cline { 2 - 4 } $0-0.5$ & $2717(1157,1560)$ & $4368(1074,3294)$ & $708(2231,4854)$ \\
$\geq 0.5$ & $888(367,521)$ & $1562(423,1139)$ & $2450(790,1660)$ \\
Unknown & $207(38,169)$ & $636(207,429)$ & $843(245,598)$ \\
Total & $3812(1562,2250)$ & $6566(1704,4862)$ & $10378(3266,7112)$ \\
& & Nagasaki & \\
$0-0.5$ & $586(266,320)$ & $968(308,660)$ & $1554(574,980)$ \\
$\geq 0.5$ & $381(171,210)$ & $741(237,504)$ & $1122(408,714)$ \\
Unknown & $153(65, \quad 88)$ & $430(155,275)$ & $583(220,363)$ \\
Total & $1120(502,618)$ & $2139(700,1439)$ & $3259(1202,2057)$ \\
\hline
\end{tabular}

*Total number of persons: (male, female) 
Tumor-registry accessions (medical records for malignant tumors only)

January-December 1995

\begin{tabular}{lccccc}
\hline & \multicolumn{4}{c}{ LSS sample } & \multirow{2}{*}{$F_{3}$, in utero } \\
\cline { 3 - 4 } City & Total & Total & AHS & samples & Others \\
\hline Hiroshima & 12,719 & 1199 & 283 & 129 & 11,391 \\
Monthly average & 1059.9 & 99.9 & 23.6 & 10.8 & 949.3 \\
Nagasaki & 5406 & 182 & 50 & 17 & 5207 \\
Monthly average & 450.5 & 15.2 & 4.2 & 1.4 & 433.9 \\
\hline
\end{tabular}

Note: $F_{1}=$ children of atomic-bomb survivors.

Tissue-registry accessions

\begin{tabular}{ccc}
\hline Year & Hiroshima & Nagasaki \\
\hline 1973 & 3249 & - \\
1974 & 5735 & 2543 \\
1975 & 4653 & 3287 \\
1976 & 6906 & 3565 \\
1977 & 9840 & 4318 \\
1978 & 8219 & 4528 \\
1979 & 10056 & 4633 \\
1980 & 10521 & 6110 \\
1981 & 12434 & 6972 \\
1982 & 13440 & 7163 \\
1983 & 13942 & 7567 \\
1984 & 14959 & 8237 \\
1985 & 15353 & 10022 \\
1986 & 17542 & 7761 \\
1987 & 20132 & 8458 \\
1988 & 21641 & 8368 \\
1989 & 22965 & 6124 \\
1990 & 25507 & 4833 \\
1991 & 26158 & 4888 \\
1992 & 30368 & 5051 \\
1993 & 34320 & 2749 \\
1994 & 30312 & 4819 \\
1995 & 37193 & 11350 \\
\hline
\end{tabular}

Note: Tissue registration was commenced in Hiroshima in April 1973 and in Nagasaki in September 1974 with funds provided by the US National Cancer Institute. 


\section{Agreements on RERF International Collaborations}

RERF has been participating in a number of international collaborative programs. The agreements on epidemiologic studies of the Techa River cohort and of the Mayak cohorts in the Russian Federation were entered into on 14 November 1995 between the RERF and the Urals Research Center for Radiation Medicine, Chelyabinsk, and the Branch N 1 of the State Scientific Center Institute of Biophysics, Russian Federation Ministry of Health and Medical Industry, respectively. These are subcontracts of the collaborative project between US NCI and the government of the Russian Federation.

\section{AGREEMENT No. \\ RERF-95-001}

\section{A GRE EMENT}

THIS AGREEMENT is entered into by and between the Radiation Effects Research Foundation, hereinafer called RERF, a non-profit foundation organized under the laws of Japan and having its principal officc in Hiroshima, Hiroshima Prefecture, Japan and the Urals Research Institutc for Radiation Medicine a non-profit governmental organization having its principal office in Chelyabinsk, Russian Federation.

\section{WI T NESS E T H:}

WHEREAS, RERF has received specified funds from the National Cancer Institute through Contract No. NO1-CP-51025, which provides for epidemiologic studies of the Techa River Cohort in the Russian Federation.

NOW, THEREFORE, the partics bereby agree and covenant as follows:

ARTICLE 1. This Agreement shall be effective as of September 29, 1995 and shall extend through September 28 , 1998 unless otherwise amended by the mutual agreement of both parties.

ARTICLE 2. The Urals Rescarch Center for Radiation Medicine (URCRM), shall furnish necessary personnel, services and materials and do all otber things neccssary to perform satisfactorily the work described herein and to report upon such work. The URCRM will perform the following tasks"

(1) Extend mortality follow-up of the Techa River cohort through the end of 1992.

(2) Evaluate the feasibility of establishing a fixed non-exposed comparison population for the Techa River cohort.

(3) Establish and maintain quality control procedures to assure standardization and a high level of quality for the collected data.

(4) Collaborate with NCI and RERF investigators in the analysis and summary of the data. Publication of results shall involve authors from the three collaborating institutions $(\mathrm{NCl}$, RERF, and URCRM). 


\section{Agreement No. RERF-95-001 (continued)}

ARTICLE 3. By no later than (a) January 15, 1996, (b) the end of each succeeding 3 month period during the term of the contract, and (c) September 15, 1998, the URCRM shall submit to the RERF a report, that will describe the progress of work conducted during the period reported, projections for the following period, and problems encountered, if any. Such reports will be written in English. These reports will be used as the basis of the quarterly and annual reports that the RERF must submit to NCl.

ARTICLE 4: It is understood by both partics that the work carried out by the URCRM under this agreement is undertaken within the framcwork of the January 1994 "Agreement Benween the Government of the United States of Americe and the Government of the Russian Federation on Cooperation in Research on Radiation Effects for the Purpose of Mininizing the Consequences of Radioactive Contamination on Health and the Environment." In particular, this work corresponds to Project 1.26 described in the February 1995 Memorandum of the Second Meeting of the Executive Committec of the Joint Coordinating Committee for Radiation Effects Research.

ARTICLE 5. Funds allotted to this Agreement total \$208,504 distributed as described in Attaclument $A$, which is cstimated to cover performance under this agreement from September 29, 1995 through September 28,1998 . The URCRM shall submit quarterly financial reports which shall include a breakdown of expenditures in fonn shown as attachments B. Reports arc to be submitted not more often than I5 days after the end of the period covered by the report. In addition, upon the completion of a report on plans for this project during the first quarter of this project, the URCRM may submit an interim financial report which can be used by the RERF to secure initial funding from $\mathrm{NCI}$.

The URCRM shall maintain adequate books and records. The URCRM agrecs to make such books and records available for an audit which may be made during the period of this agrecment cither by the RERF or by an independent firm of auditors which is acceptable to the RERF.

ARTICLE 6. Expenditure of funds provided by this Agreement shall be in accordance with the terms of this Agreement, as well as with policics and procedures of the URCRM. Notwithstanding the above, under the terms of the RERF's prime contract with the National Cancer Institute, this subcontract is subject to written authorization by the Government's Contracting OMicer for those items as set forth below. The subcontraclor agrees to enter into such further negotiations and exccutc such further instruments as may be necessary to efrect such approval. Authorizations will be passed from the US govenment via written approval from the RERF. Unless authorized in wriling, no expenditures shall be made for the following purposes:

a. Purchase or lease of any item of general purpose office furniture or office equipment regardless of dollar value (General purpose equipment is defined as any items of personal property which are usable for purposes other than research, such as office equipment and furnishings, pocket calculators, etc.);

b. Travel to attend scientific meetings

c. Patient Care Costs.

d. Capitalized non-expendable equipment (defined as having an acquisition cost of $\$ 500$ or more and a life expectancy of two years or more.

e. Foreign Travel - Request for foreign travel must be submitted at lcast cight weeks in advance, and shall contain the following: (a) Mecting(s) and place(s) to be visited, with costs and dates; (b) names and tities of personnel to travel, their functions in the project; (c) contract purposes to be served by the travel; (d) how travel of the personnel will benefit and contribute to accomplishing the project; and (f) what additional functions may be performed by the travelers to accomplish other purposes of the contract and thus further benefit the project. 


\section{Agreement No. RERF-95-001 (continued)}

ARTICLE 7. This Agreement may be terminated for convenience in whole or in part, if both parties agree that the continuation of this Agrecment will not produce beneficial results commensurate with the further cxpenditurc of funds. Both parties shall agree upon termination conditions including the effective date, and in the case of partial termination, the portion to be terminated. The Agreement to terminate shall be set forth in letter from the RERF to the URCRM. This Agreement also may be terminated for convenience unilaterally by the RERF if the prime contract under which this Agreement is issued should be terminated by the National Cancer Institutc.

IN WITNESS WHEREOF, the parties have caused this Agreement to be signed and sealed, intending to be legally bound thereby.

\section{RADIATION EFFECTS RESEARCH FOUNDATION}

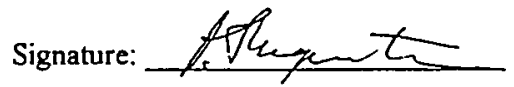

Name: Itsuzo Shigematsu, M.D.

Title: Chairman

Date: 14 November 1995

\author{
URALS RESEARCH CENTER FOR \\ RADIATION MEDICINE \\ Signature: \\ Name: Alexander V. Akleyev, $M D$ \\ Title: Director \\ Date: 26 October, 1995
}

\section{AGREEMENT No. RERF-95-002}

\section{A GREEM ENT}

THIS AGREEMENT is entercd into by and between the Radiation Effects Research Foundation, hercinafter called RERF, a non-profit foundation organized under the laws of Japan and having its principal office in Hiroshima, Hiroshima Prefecture, Japan and Branch N 1 of the State Scientific Center Institute of Biophysics, an institute of the Russian Federation Ministry of Health and Medical Industry Branch N 1, hereinafter called FIB-1, is a state, non-profit research, medical, and diagnostic institute, which operates under the laws of the Russian Federation having its principal office in Ozyorsk, Russian Federation.

\section{W ITNESSETH:}

WHEREAS, RERF has received specified funds from the National Cancer Institute through Contract No NO1-CP-51025, which provides for epidemiologic studies of the Mayak Cohorts in the Russian Federation.

NOW, THEREFORE, the parties hereby agrec and covenant as follows:

ARTICLE 1. This Agreement shall be effective as of September 29, 1995 and shall extend through September 28, 1998 unless otherwise amended by the mutual agreement of both parties. 


\section{Agreement No. RERF-95-002 (continued)}

ARTICLE 2. Branch N 1, Institute of Biophysics(FIB-1), shall furnish necessary personnel, services and materials and do all other things necessary to perform satisfactorily the work described herein and to report upon such work. The FIB-1 will perform the following tasks:

(1) Develop improved data bases for the Mayak cohorts

(2) Obtain death certificates for Mayak workers who have migrated from the study area.

(3) Continue tracing the Mayak children's cohort so that cancer mortality can be evaluated.

(4) Evaluate the feasibility of establishing a non-exposed comparison group for the Mayak worker cohort.

(5) Evaluate the feasibility of a thyroid cancer study in a subcohort of the Mayak children's cohort and of studying parental radiation dose as a risk factor for childhood cancer.

(ด) Establish and maintain quality control procedures to assure standardization and a high level of quality for the collected data.

(T) Collaboratc with $\mathrm{NCI}$ and RERF investigators in the analysis and summary of the data. Publication of results shall involve authors from the three collaborating institutions $(\mathrm{NCl}$, RERF, and FBB-1).

ARTICLE 3. By no later than (a) January 15,1996 , (b) the end of each succeeding 3 month period during the term of the contract, and (c) September 15, 1998, the FIB-1 shall submit to the RERF a report, that will describe the progress of work conducted during the period reported, projections for the following period, and problems encountered, if any. Such reports will be written in English. These reports will be used as the basis of the quarterly and annual reports that the RERF must submit to NCL

ARTICLE 4. Funds allotted to this Agreement total \$205,306 distributed as described in Altachment A, which is estimated to cover performance under this agrecment from September 29, 1995 through September 28, 1998. The FIB-1 shall submit quarterly financial reports which shall include a breakdown of expenditures in the form shown as attachment $B$. Reports are to be submitted not more often than 15 days after the end of the period covered by the report. In addition, upon the completion of a report on plans for this project during the first quarter of this project, the FIB-1 may submit an interim financial report which can be used by the RERF to secure initial funding from NCI.

The FIB-1 shall maintain adequate books and records. The FIB-1 agrees to make such books and records available for audits which may be made during the period of this agreement either by the RERF or by an independent firm of auditors which is acceptable to the RERF.

ARTICLE 5. Expenditure of funds provided by this Agreement shall be in accordance with the terms of this Agrecment, as well as with policies and procedures of the FiB-1. Notwithstanding the above, under the terms of the RERF's prime contract with the National Cancer Institute, this subcontract is subject to written authorization by the Govemment's Contracting Officer for those itcms as set forth below. The subcontractor agrees to enter into such further negotiations and execute such further instruments as may be necessary to effect such approval. Authorizations will be passed from the US government via written approval from the RERF. Unless authorized in writing, no expenditures shall be made for the following purposes:

a. Purchase or lease of any item of general purpose office furniture or office equipment regardless of dollar value (Gencral purpose equipment is defined as any items of personal property which are usable for purposes other than research, such as office cquipment and furnishings, pocket calculators, etc.);

b. Travel to attend scientific meetings

Continued on next page 


\section{Agreement No. RERF-95-002 (continued)}

c Patient Care Costs.

d. Capitalized non-expendable equipment (defined as having an acquisition cost of $\$ 500$ or more and a life expectancy of two years or more).

e. Forcign Travel - Request for forcign travel must be submitted at least cight weeks in advance, and shall contain the following (a) Meeting(s) and place(s) to be visitcd, with costs and dates; (b) names and titles of personnel to travel, their functions in the project, (c) contract purposes to be served by the travel; (d) how travel of the personnel will benefit and contribute to accomplishing the project; and (D) what additional functions may be performed by the travelers to accomplish other purposes of the contract and thus further benefit the project.

ARTICLE 6. This Agreement may be terminated for convenience in whole or in part, if both parties agree that the continuation of this $\Lambda$ greement will not produce beneficial results commensurate with the further expenditure of funds. Both parties shall agree upon termination conditions including the effective date, and in the case of partial termination, the portion to be terminated. The Agrecnent to terminate shall he set forth in letter from the RERF to the FIB-1. This $\Lambda$ grecment also may be terminated for convenience unilaterally by the RERF if the prime contract under which this Agrecment is issued should be terminated by the National Cancer Institute.

IN WITNESS WHEREOF, the parties have caused this Agreement to be signed and sealed, intending to be legally bound thercby.

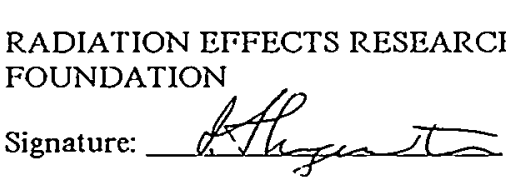

Name: Itsuzo Shigematsu, M.D.

Title:. Chairman

Date: 14 November 1995

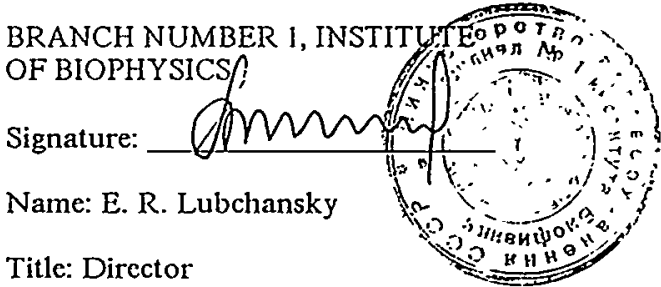

Date: 2 November 1995 


\section{Chronology of Events}

\section{April 1995-31 March 1996}

Editor's note: Unless otherwise indicated, the events listed were held at the Hiroshima Laboratory.

1995

April 4 Twenty employees commended for long service.

4 Executive Committee meeting

14 Three commended for long service, Nagasaki.

19-21 The 22nd Scientific Council meeting recommended assessment of RERF's scientific activities by an outside group of scientists.

25 Executive Committee meeting, Operating Committee meeting, staff liaison meeting

26 Section chiefs liaison meeting

27 Laboratory liaison meeting, Nagasaki

May $16 \quad$ Executive Committee meeting

24-25 Research audit

26 Research audit, Nagasaki

June $\quad 1$ Laboratory liaison meeting, Nagasaki

6 Executive Committee meeting

6-7 July Departure of the 10th medical team to examine A-bomb survivors living in the United States. Kazuo Neriishi, chief, Division of Medicine, Department of Clinical Studies, and Tadaaki Watanabe, General Affairs Unit supervisor, General Affairs Section, Secretariat, participated.

20 Executive Committee meeting, Operating Committee meeting, staff liaison meeting

20 Retirement ceremony for the first half of Fiscal Year 1995 (13 retirees in Hiroshima)

22 Laboratory liaison meeting, Nagasaki

23 Retirement ceremony for the first half of Fiscal Year 1995 (13 retirees in Nagasaki)

26-28 29th meeting of the RERF Board of Directors: DOE's contract with NAS deferred for two years as recommended by the Scientific Council. Holding of a blue ribbon panel meeting approved.

28 Ceremony and commemorative lectures for the 20th anniversary of RERF (Minami-ward Culture Center Hall) 
June 29 Ceremony and commemorative lectures for the 20th anniversary of RERF (Nagasaki Town and Village Hall)

30 12th exchange seminar of National Institute of Radiological Sciences, RERF and Research Institute for Radiation Biology and Medicine (Chiba)

July $\quad 3$ Vice Chairman Mortimer L Mendelsohn retired.

4 Executive Committee meeting

18 Executive Committee meeting, Operating Committee meeting, staff liaison meeting

19 Section chiefs liaison meeting

20 Laboratory liaison meeting, Nagasaki

20 Inspection by the Hiroshima Legal Affairs Bureau

August 5 1st RERF Open House held as a commemorative event of the 20th anniversary of RERF, with some 900 visitors.

6 Chairman Itsuzo Shigematsu and Chief of Research Donald $R$ Harkness attended Atomic Bomb Peace Memorial Ceremony.

6 Chairman Itsuzo Shigematsu attended memorial ceremony held by the Hiroshima City Medical Association for its members and medical workers who fell victim to the atomic bombing.

9 Permanent Director Yutaka Hasegawa, Chief of Research Donald R Harkness and Chief of Administration Tadashi Nakaoka attended Nagasaki City Atomic Bomb Peace Memorial Ceremony.

9 Assistant Division Chief of Medicine Masazumi Akahoshi attended memorial ceremony sponsored by Nagasaki University School of Medicine.

9 Two videos shown to commemorate the anniversary of the atomic bombing of Nagasaki.

29 Executive Committee meeting, Operating Committee meeting, staff liaison meeting

29 Chief of Research Donald R Harkness retired.

30 Section chiefs liaison meeting

31 Laboratory liaison meeting, Nagasaki

September 9 Seymour Abrahamson assumed vice chairmanship and permanent directorship (chief of research).

12 Executive Committee meeting

26 Executive Committee meeting, Operating Committee meeting, staff liaison meeting

27 Section chiefs liaison meeting 
September 28 Laboratory liaison meeting, Nagasaki

29 Study meeting on emergency treatment held for the 46th National Labor Health Week.

October 9-10 Several RERF researchers participated in the 1st Schull International Symposium "A-bomb Survivors in Hiroshima and Nagasaki and Their Children-in Commemoration of 50 Years of Research," Houston, Texas.

14 Nagasaki employees' recreational overnight trip to Fukuoka Dome

17 Executive Committee meeting, Operating Committee meeting, staff liaison meeting

18 Section chiefs liaison meeting

19 Laboratory liaison meeting, Nagasaki

23-25 6th Radiation Emergency Medical Preparedness and Assistance Network (REMPAN) meeting

25 Inspection by the Legal Affairs Bureau

28 Hiroshima employees' recreational one-day trip to Seto Ohashi bridge and its vicinities

November 21995 seminar of physicians of designated medical institutions for A-bomb survivors, Hiroshima Prefectural Culture Center.

10 Permanent Director to Oversee Nagasaki Laboratory Yutaka Hasegawa attended Nagasaki University Medical School's Autopsy Memorial Service.

14 Executive Committee meeting, Operating Committee meeting, staff liaison meeting

15 Section chiefs liaison meeting

16 Laboratory liaison meeting, Nagasaki

December 7 Evacuation training, Nagasaki

14 Retirement ceremony for the last half of Fiscal Year 1995 (5 retirees in Nagasaki)

18 Meeting of research department chiefs

19 Executive Committee meeting, Operating Committee meeting, staff liaison meeting

19 Retirement ceremony for the last half of Fiscal Year 1995 (3 retirees in Hiroshima)

20 Section chief liaison meeting

21 Laboratory liaison meeting, Nagasaki 
1996

January 9 Executive Committee meeting

22 Meeting of research department chiefs

23 Executive Committee meeting, Operating Committee meeting, staff liaison meeting

24 Section chiefs liaison meeting

25 Laboratory liaison meeting, Nagasaki

February 4-7 Blue Ribbon Panel meeting: hearing and discussion held concerning the future of RERF research studies.

8 Blue Ribbon Panel meeting: hearing and discussion held concerning the future of RERF research studies, Nagasaki.

19 Meeting of research department chiefs

19 Meeting of Shigematsu Study Group, Hiroshima Kousei Nenkin Hall

20 Executive Committee meeting, Operating Committee meeting, staff liaison meeting

21 Section chiefs liaison meeting

22 Laboratory liaison meeting, Nagasaki

March 12 Executive Committee meeting

26 Executive Committee meeting, Enlarged Operating Committee meeting

28 Laboratory liaison meeting, Nagasaki

29 Section chiefs liaison meeting 